\title{
Control-centric Modelling and Nonlinear Control of the Blade Sailing Phenomenon
}

by

\section{Mohammad Riazi}

\author{
A thesis submitted to \\ the Faculty of Graduate Studies and Research \\ in partial fulfilment of \\ the requirements for the degree of \\ Master of Applied Science \\ in
}

\begin{abstract}
Mechanical Engineering
Ottawa-Carleton Institute for Mechanical and Aerospace Engineering

Department of Mechanical and Aerospace Engineering

Carleton University

Ottawa, Ontario, Canada
\end{abstract}

September 2012

Copyright (C)

2012 - Mohammad Riazi 
Library and Archives

Canada

Published Heritage

Branch

395 Wellington Street

Ottawa ON K1A ON4

Canada
Bibliothèque et

Archives Canada

Direction du

Patrimoine de l'édition

395 , rue Wellington

Ottawa ON K1A ON4

Canada
Your file Votre référence

ISBN: 978-0-494-93502-6

Our file Notre référence

ISBN: $978-0-494-93502-6$
NOTICE:

The author has granted a nonexclusive license allowing Library and Archives Canada to reproduce, publish, archive, preserve, conserve, communicate to the public by telecommunication or on the Internet, loan, distrbute and sell theses worldwide, for commercial or noncommercial purposes, in microform, paper, electronic and/or any other formats.

The author retains copyright ownership and moral rights in this thesis. Neither the thesis nor substantial extracts from it may be printed or otherwise reproduced without the author's permission.
AVIS:

L'auteur a accordé une licence non exclusive permettant à la Bibliothèque et Archives Canada de reproduire, publier, archiver, sauvegarder, conserver, transmettre au public par télécommunication ou par l'Internet, prêter, distribuer et vendre des thèses partout dans le monde, à des fins commerciales ou autres, sur support microforme, papier, électronique et/ou autres formats.

L'auteur conserve la propriété du droit d'auteur et des droits moraux qui protege cette thèse. $\mathrm{Ni}$ la thèse ni des extraits substantiels de celle-ci ne doivent être imprimés ou autrement reproduits sans son autorisation.
In compliance with the Canadian Privacy Act some supporting forms may have been removed from this thesis.

While these forms may be included in the document page count, their removal does not represent any loss of content from the thesis.
Conformément à la loi canadienne sur la protection de la vie privée, quelques formulaires secondaires ont été enlevés de cette thèse.

Bien que ces formulaires aient inclus dans la pagination, il n'y aura aucun contenu manquant. 


\section{Abstract}

A reduced control-centric model of a particular behaviour associated with maritime helicopter rotor systems, known as Blade Sailing Phenomenon (BSP), is developed. BSP is a transient aeroelastic phenomenon described by the large undesired flapping motion of the helicopter rotor blades during low rotor speeds under high wind and elevated sea conditions. The developed model utilizes the Unified Airloads Model to capture the aerodynamic loads and the Intrinsic Nonlinear Beam Model to capture the structural behaviour of the blade. Integrally Actuated Twist (IAT) is used as the actuation strategy and a nonlinear feedback controller based on the Lyapunov stability theorem for autonomous dynamical systems is developed to counter the BSP.

To evaluate the performance of the designed controller, the reduced BSP model is then discretized in the space domain using the Galerkin Spectral Discretization Method. The results prove the promising potential of the designed controller in countering the BSP in the reduced model. A further assessment of the controller is carried out by testing its performance in a comprehensive BSP model validated against experimental data. The results prove the capability of the controller to counter the BSP in a more accurate blade model and a more realistic simulation of a maritime environment. 
I would like to dedicate my work to my father who I owe all my life achievements to, and who passed away while I was working on this research, and who I did not get a chance to visit in the last two years of his life and to attend his funeral. 


\section{Acknowledgments}

My first thanks go to my wife Dr. Parvaneh Fallah who stood by me, encouraged me, and supported me for four years. I will never forget her patience and motivation during the last two years. I would also like to acknowledge the guidance, advice, and supervision of my supervisors: Prof. Fred F. Afagh and Prof. Robert G. Langlois. I sincerely thank them for their trust in my capabilities and I feel fortunate to have benefited from their expertise. During this research, I have received a number of technical comments from some professors and researchers. I would like to thank Prof. David A. Peters of the Washington Univeristy in Saint Louis for his valuable comments in using the Unified Airloads Model for this research. Thanks are due to Dr. Zahra Sotoudeh of the Virgina Polytechnic Institute and State University for her advice on applying the intrinsic beam equations to this research, and Dr. Matthias Althoff of Carnegie Mellon University for his computer programming comments on the Galerkin discretization method. Last but not least, I thank Prof. Mojtaba Ahmadi from Carleton University for his productive advice on using an energy-based controller in this research. 


\section{Table of Contents}

$\begin{array}{lll}\text { Abstract } & \text { ii }\end{array}$

Acknowledgments iv

Table of Contents $\quad \mathbf{v}$

List of Symbols $\quad$ viii

1 Introduction 1

1.1 Blade Sailing Phenomenon Definition . . . . . . . . . . . 2

1.2 Literature Review . . . . . . . . . . . . . . . . . 3

1.2 .1 Aerodynamics . . . . . . . . . . . . . . . 3

1.2.2 BSP Control and Modelling for Control . . . . . . . . . 5

1.3 Motivation and Objective $\ldots \ldots \ldots \ldots \ldots$

1.4 Overview of the Present Research . . . . . . . . . . . . . 11

2 Aerodynamic Loads Modelling 12

2.1 Unified Airloads Model . . . . . . . . . . . . . . . . . . . 12

2.1.1 Unstalled Generalized Loads . . . . . . . . . . . . . . 13

2.1 .2 Induced Flow $\ldots \ldots \ldots \ldots \ldots \ldots$

2.1 .3 Static Corrections . . . . . . . . . . . . . . . 17

2.1 .4 Dynamic Stall . . . . . . . . . . . . . . . 18 


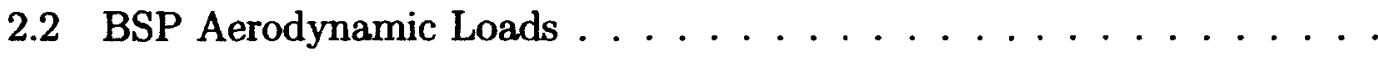

3 Dynamic Modelling $\quad 27$

3.1 Structural Modelling . . . . . . . . . . . . . . 27

3.1.1 Intrinsic Structural Dynamic Equations . . . . . . . . . . 28

3.1.2 Constitutive Relations .................. 31

3.2 Actuation Loads . . . . . . . . . . . . . . . . 32

3.3 External Loads . . . . . . . . . . . . . . . . . 35

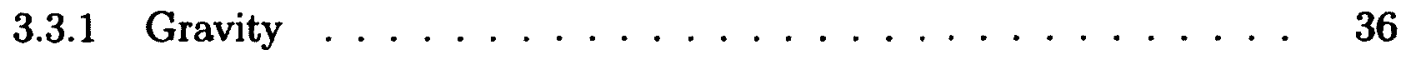

3.3.2 Structural Damping . . . . . . . . . . . . . . 37

3.3.3 Aerodynamics ................... 38

3.3.4 Aerodynamic Inflow . . . . . . . . . . . . . . 41

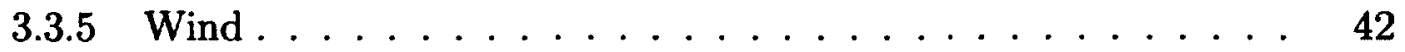

3.3.6 Boundary Conditions . . . . . . . . . . . . . . 44

3.4 Total Governing Equations . . . . . . . . . . . . . . . 45

4 Control System Design $\quad 49$

4.1 Lyapunov Stability for Autonomous Dynamical Systems . . . . . . 50

4.1.1 Definitions ....................... 50

4.1.2 Lyapunov Stability Theorem for Autonomous Systems . . . . 51

4.2 Lyapunov Stability for Non-autonomous Systems . . . . . . . . . . . 52

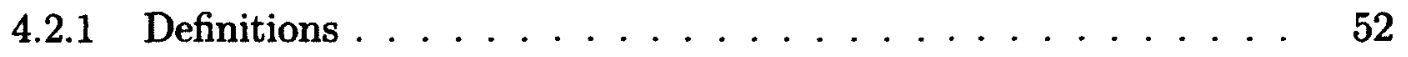

4.2.2 Lyapunov Stability Theorems for Non-autonomous Systems . 54

4.3 Candidate BSP Lyapunov Function . . . . . . . . . . . . . 55

4.3.1 Energy Balance Equation ............... 56

4.3.2 Energy Consistent Weighting . . . . . . . . . 58

4.4 The BSP Lyapunov Controller . . . . . . . . . . . . . . . . . . 64 
5 Galerkin Spectral Discretization

5.1 The Galerkin Method Concept . . . . . . . . . . . . . . 72

5.2 Weighting Functions $\ldots \ldots \ldots \ldots \ldots \ldots$

5.3 Comparison Functions $\ldots \ldots \ldots \ldots \ldots \ldots$

5.4 State Equations . . . . . . . . . . . . . . . 79

5.5 Output Equations . . . . . . . . . . . . . . . . . 90

6 Results and Discussion $\quad 97$

6.1 Galerkin Method Validation . . . . . . . . . . . . . . 97

6.2 Controller Effectiveness in the Reduced BSP Model . . . . . . . . 103

6.3 Controller Effectiveness in the Full BSP Model . . . . . . . . . . . 105

7 Discussion and Conclusion $\quad 117$

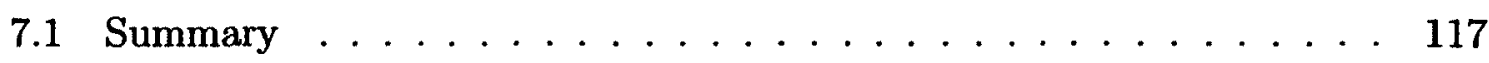

7.2 Contributions . . . . . . . . . . . . . . . . 119

7.3 Future work . . . . . . . . . . . . . . . . 119

List of References 122

Appendix A Aerodynamic Modelling $\quad 126$

A.1 Unstalled Generalized Loads . . . . . . . . . . . . . . . . . 126

$\begin{array}{ll}\text { Appendix B Dynamic Modelling } & 131\end{array}$

B.1 Aerodynamic Coefficient Matrices . . . . . . . . . . . . . 131

Appendix C Galerkin Discretization $\quad 136$

C.1 Approximation of State Variables . . . . . . . . . . 136

C.2 Matrices and Vectors Used in Discretization . . . . . . . . . . 139 


\section{List of Symbols}

\section{Chapter 2}

\begin{tabular}{|c|c|c|c|}
\hline Symbol & Size & SI Unit & Definition \\
\hline$h(x, t)$ & $1 \times 1$ & $\mathbf{m}$ & $\begin{array}{l}\text { Airfoil deformation in its reference } \\
\text { frame }\end{array}$ \\
\hline$u_{0}$ & $1 \times 1$ & $\mathrm{~m} \cdot \mathrm{s}^{-1}$ & $\begin{array}{l}\text { Air velocity in positive } x \text { direction of } \\
\text { the airfoil reference frame }\end{array}$ \\
\hline$v_{0}$ & $1 \times 1$ & $\mathrm{~m} \cdot \mathrm{s}^{-1}$ & $\begin{array}{l}\text { Air velocity in positive } y \text { direction of } \\
\text { the airfoil reference frame }\end{array}$ \\
\hline$v_{1}$ & $1 \times 1$ & $\mathrm{~s}^{-1}$ & $\begin{array}{l}\text { Air velocity gradient in the airfoil ref- } \\
\text { erence frame }\end{array}$ \\
\hline$b$ & $1 \times 1$ & $\mathbf{m}$ & Airfoil (blade cross section) semi-chord \\
\hline$\rho$ & $1 \times 1$ & $\mathrm{~kg} \cdot \mathrm{m}^{-3}$ & Air density \\
\hline$M$ & $1 \times 1$ & -- & $\begin{array}{l}\text { Number of generalized airfoil motions } \\
\text { minus } 1\end{array}$ \\
\hline$\left\{h_{n}\right\}$ & $(M+1) \times 1$ & --- & Generalized airfoil motions \\
\hline
\end{tabular}




\begin{tabular}{|c|c|c|c|}
\hline$\left\{L_{n}\right\}$ & $(M+1) \times 1$ & -- & Generalized airfoil loads \\
\hline$\left\{v_{n}\right\}$ & $(M+1) \times 1$ & --- & Generalized velocity \\
\hline$\lambda_{n}$ & $1 \times 1$ & $\mathrm{~m} \cdot \mathrm{s}^{-1}$ & $\begin{array}{l}\text { The } n^{\text {th }} \text { inflow state or the } n^{\text {th }} \text { coeffi- } \\
\text { cient of the induced flow }\end{array}$ \\
\hline$\Gamma$ & $1 \times 1$ & $\mathrm{~m}^{2} \cdot \mathrm{s}^{-1}$ & Total bound circulation on the airfoil \\
\hline$\Gamma_{d s_{0}}$ & $1 \times 1$ & $\mathrm{~m}^{2} \cdot \mathrm{s}^{-1}$ & $\begin{array}{l}\text { Circulation loss of the airfoil during dy- } \\
\text { namic stall }\end{array}$ \\
\hline$\Gamma_{d s_{n}}$ & $1 \times 1$ & $\mathrm{~m}^{2} \cdot \mathrm{s}^{-1}$ & $\begin{array}{l}\text { Dynamic stall state corresponding to } \\
\text { the } n^{\text {th }} \text { generalized load }\end{array}$ \\
\hline$\zeta_{n}$ & $1 \times 1$ & $\mathrm{~m} \cdot \mathrm{s}^{-1}$ & $\begin{array}{l}\text { Induced velocity at the airfoil produced } \\
\text { from some bound circulation }\end{array}$ \\
\hline$D$ & $1 \times 1$ & $\mathrm{~kg} \cdot \mathrm{s}^{-2}$ & Airfoil induced drag per unit span \\
\hline$[K]$ & $\begin{array}{l}(M+1) \times \\
(M+1)\end{array}$ & --- & Aerodynamic stiffness matrix \\
\hline$[C]$ & $\begin{array}{l}(M+1) \times \\
(M+1)\end{array}$ & --- & Aerodynamic damping matrix \\
\hline$[G]$ & $\begin{array}{l}(M+1) \times \\
(M+1)\end{array}$ & --- & Greenberg matrix \\
\hline$[S]$ & $\begin{array}{l}(M+1) \times \\
(M+1)\end{array}$ & --- & Drag matrix \\
\hline
\end{tabular}




\begin{tabular}{|c|c|c|c|}
\hline$[M]$ & $\begin{array}{l}(M+1) \times \\
(M+1)\end{array}$ & --- & Aerodynamic mass matrix \\
\hline$[H]$ & $\begin{array}{l}(M+1) \times \\
(M+1)\end{array}$ & --- & Aerodynamic coefficient matrix \\
\hline$\alpha$ & $1 \times 1$ & rad & Airfoil angle of attack \\
\hline$b_{n}$ & $1 \times 1$ & --- & The $n^{\text {th }}$ inflow state coefficient \\
\hline$c l_{\alpha}$ & $1 \times 1$ & --- & Slope of the airfoil lift coefficient curve \\
\hline$c l_{0}$ & $1 \times 1$ & --- & Lift coefficient at zero angle of attack \\
\hline$\left.c m_{\alpha}\right|_{M C}$ & $1 \times 1$ & --- & $\begin{array}{l}\text { Slope of the pitching moment curve at } \\
\text { mid-chord }\end{array}$ \\
\hline$\left.c m_{\alpha}\right|_{C R}$ & $1 \times 1$ & -- & $\begin{array}{l}\text { Slope of the pitching moment curve at } \\
\text { the centre of rotation }\end{array}$ \\
\hline$c m_{0}$ & $1 \times 1$ & --- & $\begin{array}{l}\text { Pitching moment coefficient at zero an- } \\
\text { gle of attack }\end{array}$ \\
\hline$a$ & $1 \times 1$ & --- & $\begin{array}{l}\text { Distance between the airfoil center of } \\
\text { rotation and its mid-chord per unit } \\
\text { chord }\end{array}$ \\
\hline$a_{C O R}$ & $1 \times 1$ & -- & $\begin{array}{l}\text { Corrected distance between the airfoil } \\
\text { center of rotation and its mid-chord per } \\
\text { unit chord }\end{array}$ \\
\hline$f_{L}$ & $1 \times 1$ & --- & Airfoil lift coefficient correction factor \\
\hline
\end{tabular}




\begin{tabular}{|c|c|c|c|}
\hline$f_{M}$ & $1 \times 1$ & --- & $\begin{array}{l}\text { Airfoil pitching moment coefficient cor- } \\
\text { rection factor }\end{array}$ \\
\hline$u_{T}$ & $1 \times 1$ & $\mathrm{~m} \cdot \mathrm{s}^{-1}$ & Total air velocity over the airfoil \\
\hline$\omega_{d s}$ & $1 \times 1$ & --- & Dynamic stall frequency parameter \\
\hline$\eta_{d s}$ & $1 \times 1$ & --- & Dynamic stall damping parameter \\
\hline$e_{d s}$ & $1 \times 1$ & --- & Dynamic stall time delay parameter \\
\hline$\Delta c_{n}$ & $1 \times 1$ & --- & $\begin{array}{l}\text { Static residual of the } n^{\text {th }} \text { generalized } \\
\text { load }\end{array}$ \\
\hline$\Delta c l$ & $1 \times 1$ & --- & Static residual of the airfoil lift \\
\hline$\Delta c d$ & $1 \times 1$ & --- & Static residual of the airfoil drag \\
\hline$\Delta c m$ & $1 \times 1$ & --- & $\begin{array}{l}\text { Static residual of the airfoil pitching } \\
\text { moment }\end{array}$ \\
\hline$L_{T_{n}}$ & $1 \times 1$ & --- & $\begin{array}{l}\text { The } n^{\text {th }} \text { generalized airfoil total load in- } \\
\text { cluding dynamic stall }\end{array}$ \\
\hline$D_{T}$ & $1 \times 1$ & $\mathrm{~kg} \cdot \mathrm{s}^{-2}$ & $\begin{array}{l}\text { Airfoil total drag including dynamic } \\
\text { stall per unit span }\end{array}$ \\
\hline${ }^{B} f^{a}$ & $3 \times 1$ & $\mathrm{~kg} \cdot \mathrm{s}^{-2}$ & $\begin{array}{l}\text { Cross sectional aerodynamic forces de- } \\
\text { fined in the deformed structural frame }\end{array}$ \\
\hline${ }^{B} m^{a}$ & $3 \times 1$ & $\mathrm{~kg} \cdot \mathrm{m} \cdot \mathrm{s}^{-2}$ & $\begin{array}{l}\text { Cross sectional aerodynamic moments } \\
\text { defined in the deformed structural } \\
\text { frame }\end{array}$ \\
\hline
\end{tabular}




\section{Chapter 3}

\begin{tabular}{|c|c|c|c|}
\hline Symbol & Size & SI Unit & Definition \\
\hline$F$ & $3 \times 1$ & $\mathrm{~kg} \cdot \mathrm{m} \cdot \mathrm{s}^{-2}$ & Internal force \\
\hline$M$ & $3 \times 1$ & $\mathrm{~kg} \cdot \mathrm{m}^{2} \cdot \mathrm{s}^{-2}$ & Internal moment \\
\hline$F^{\text {act }}$ & $3 \times 1$ & $\mathrm{~kg} \cdot \mathrm{m} \cdot \mathrm{s}^{-2}$ & Actuation force \\
\hline$M^{\text {act }}$ & $3 \times 1$ & $\mathrm{~kg} \cdot \mathrm{m}^{2} \cdot \mathrm{s}^{-2}$ & Actuation moment \\
\hline$V$ & $3 \times 1$ & $\mathrm{~m} \cdot \mathrm{s}^{-1}$ & Linear velocity \\
\hline$V_{w}$ & $3 \times 1$ & $\mathbf{m} \cdot \mathbf{s}^{-1}$ & Linear wind velocity \\
\hline$\Omega$ & $3 \times 1$ & $\mathrm{rad} \cdot \mathrm{s}^{-1}$ & Angular velocity \\
\hline$P$ & $3 \times 1$ & $\mathrm{~kg} \cdot \mathrm{s}^{-1}$ & Linear momentum per unit length \\
\hline$H$ & $3 \times 1$ & $\mathrm{~kg} \cdot \mathrm{m} \cdot \mathrm{s}^{-1}$ & Angular momentum per unit length \\
\hline$\gamma$ & $3 \times 1$ & --- & Strain \\
\hline$\kappa$ & $3 \times 1$ & $\mathrm{~m}^{-1}$ & Curvature \\
\hline$k$ & $3 \times 1$ & $\mathrm{~m}^{-1}$ & Initial curvature \\
\hline$f$ & $3 \times 1$ & $\mathrm{~kg} \cdot \mathrm{s}^{-2}$ & External force per unit length \\
\hline$m$ & $3 \times 1$ & $\mathrm{~kg} \cdot \mathrm{m} \cdot \mathrm{s}^{-2}$ & External moment per unit length \\
\hline$e_{1}$ & $3 \times 1$ & $-\cdots$ & Unit vector along axis 1 \\
\hline
\end{tabular}




\begin{tabular}{|c|c|c|c|}
\hline$e_{2}$ & $3 \times 1$ & -- & Unit vector along axis 2 \\
\hline$e_{3}$ & $3 \times 1$ & --- & Unit vector along axis 3 \\
\hline${ }^{l} e_{1}$ & $3 \times 1$ & --- & $\begin{array}{l}\text { Unit vector along axis } 1 \text { defined in an } \\
\text { inertial frame }\end{array}$ \\
\hline${ }^{I} e_{2}$ & $3 \times 1$ & --- & $\begin{array}{l}\text { Unit vector along axis } 2 \text { defined in an } \\
\text { inertial frame }\end{array}$ \\
\hline${ }^{1} e_{3}$ & $3 \times 1$ & --- & $\begin{array}{l}\text { Unit vector along axis } 3 \text { defined in an } \\
\text { inertial frame }\end{array}$ \\
\hline${ }^{B} e_{1}$ & $3 \times 1$ & --- & $\begin{array}{l}\text { Unit vector along axis } 1 \text { defined in the } \\
\text { structural deformed frame }\end{array}$ \\
\hline${ }^{B} e_{2}$ & $3 \times 1$ & --- & $\begin{array}{l}\text { Unit vector along axis } 2 \text { defined in the } \\
\text { structural deformed frame }\end{array}$ \\
\hline${ }^{B} e_{3}$ & $3 \times 1$ & --- & $\begin{array}{l}\text { Unit vector along axis } 3 \text { defined in the } \\
\text { structural deformed frame }\end{array}$ \\
\hline $\bar{m}$ & $1 \times 1$ & $\mathrm{~kg} \cdot \mathrm{m}^{-1}$ & Mass per unit length \\
\hline$\xi$ & $3 \times 1$ & $\mathbf{m}$ & Mass center offset \\
\hline$G$ & $3 \times 3$ & $\mathrm{~kg} \cdot \mathrm{m}^{-1}$ & Matrix of Mass per unit length \\
\hline$Q$ & $3 \times 3$ & $\mathrm{~kg}$ & 1st moment of inertia per unit length \\
\hline$I$ & $3 \times 3$ & $\mathrm{~kg} \cdot \mathrm{m}$ & 2nd moment of inertia per unit length \\
\hline$R$ & $3 \times 3$ & $\mathrm{~kg}^{-1} \cdot \mathrm{m}^{-1} \cdot \mathrm{s}^{2}$ & Flexibility matrix \\
\hline$S$ & $3 \times 3$ & $\mathrm{~kg}^{-1} \cdot \mathrm{m}^{-1} \cdot \mathrm{s}^{2}$ & Flexibility matrix \\
\hline
\end{tabular}




\begin{tabular}{|c|c|c|c|}
\hline$T$ & $3 \times 3$ & $\mathrm{~kg}^{-1} \cdot \mathrm{m}^{-1} \cdot \mathrm{s}^{2}$ & Flexibility matrix \\
\hline$E$ & $1 \times 1$ & $\mathrm{~V} \cdot \mathrm{m}^{-1}$ & Electric field across active fibres \\
\hline$V_{p}$ & $1 \times 1$ & V & Voltage along active fibres \\
\hline$Y^{F}$ & $3 \times 1$ & $\mathrm{~kg} \cdot \mathrm{m} \cdot \mathrm{s}^{-2} \cdot \mathrm{V}^{-1}$ & Piezoelectric field coefficient vector \\
\hline$Y^{M}$ & $3 \times 1$ & $\mathrm{~kg} \cdot \mathrm{m}^{2} \cdot \mathrm{s}^{-2} \cdot \mathrm{V}^{-1}$ & Piezoelectric field coefficient vector \\
\hline$u_{1}$ & $3 \times 1$ & $\mathrm{~kg} \cdot \mathrm{m} \cdot \mathrm{s}^{-3}$ & $\begin{array}{l}\text { Actuation force rate commanded by } \\
\text { controller }\end{array}$ \\
\hline$u_{2}$ & $3 \times 1$ & $\mathrm{~kg} \cdot \mathrm{m}^{2} \cdot \mathrm{s}^{-3}$ & $\begin{array}{l}\text { Actuation moment rate commanded by } \\
\text { controller }\end{array}$ \\
\hline$f^{g r}$ & $3 \times 1$ & $\mathrm{~kg} \cdot \mathrm{s}^{-2}$ & Gravity force per unit length \\
\hline$m^{g r}$ & $3 \times 1$ & $\mathrm{~kg} \cdot \mathrm{m} \cdot \mathrm{s}^{-2}$ & Gravity moment per unit length \\
\hline$f^{d}$ & $3 \times 1$ & $\mathrm{~kg} \cdot \mathrm{s}^{-2}$ & $\begin{array}{l}\text { Structural damping force per unit } \\
\text { length }\end{array}$ \\
\hline$m^{d}$ & $3 \times 1$ & $\mathrm{~kg} \cdot \mathrm{m} \cdot \mathrm{s}^{-2}$ & $\begin{array}{l}\text { Structural damping moment per unit } \\
\text { length }\end{array}$ \\
\hline$f^{a}$ & $3 \times 1$ & $\mathrm{~kg} \cdot \mathrm{s}^{-2}$ & Aerodynamic force per unit length \\
\hline$m^{a}$ & $3 \times 1$ & $\mathrm{~kg} \cdot \mathrm{m} \cdot \mathrm{s}^{-2}$ & Aerodynamic moment per unit length \\
\hline$g$ & $3 \times 1$ & --- & $\begin{array}{l}\text { Unit vector along negative axis } 3 \text {, de- } \\
\text { fined in the structural deformed frame }\end{array}$ \\
\hline$|g|$ & $1 \times 1$ & -- & $\begin{array}{l}\text { Magnitude of the unit vector along neg- } \\
\text { ative axis } 3\end{array}$ \\
\hline
\end{tabular}




\begin{tabular}{|c|c|c|c|}
\hline$g_{m}$ & $1 \times 1$ & $\mathrm{~m} \cdot \mathrm{s}^{-2}$ & Gravity vector magnitude \\
\hline$C$ & $6 \times 6$ & -- & $\begin{array}{l}\text { Structural damping proportionality } \\
\text { matrix }\end{array}$ \\
\hline $\bar{\eta}$ & $1 \times 1$ & --- & Structural damping adjuster \\
\hline $\bar{\delta}$ & $1 \times 1$ & --- & Structural damping adjuster \\
\hline$M=1$ & $1 \times 1$ & $-\cdots$ & $\begin{array}{l}\text { Number of chosen generalized airfoil } \\
\text { motions minus } 1\end{array}$ \\
\hline$N=3$ & $1 \times 1$ & --- & Number of chosen inflow states \\
\hline$X_{i j \ldots}$ & $3 \times 3(1)$ & --- & Aerodynamic coefficient matrix/vector \\
\hline$F_{\lambda}$ & $3 \times 3$ & $\mathrm{~kg} \cdot \mathrm{m}^{-1} \cdot \mathrm{s}^{-1}$ & $\begin{array}{l}\text { Unsteady aerodynamic force coefficient } \\
\text { matrix }\end{array}$ \\
\hline$M_{\lambda}$ & $3 \times 3$ & $\mathrm{~kg} \cdot \mathrm{s}^{-1}$ & $\begin{array}{l}\text { Unsteady aerodynamic moment coeffi- } \\
\text { cient matrix }\end{array}$ \\
\hline$\Omega_{\text {ship }}$ & $3 \times 1$ & $\mathrm{rad} \cdot \mathrm{s}^{-1}$ & $\begin{array}{l}\text { Ship angular velocity in the inertial } \\
\text { frame }\end{array}$ \\
\hline$\Omega_{h u b}$ & $3 \times 1$ & $\operatorname{rad} \cdot \mathrm{s}^{-1}$ & Hub angular velocity in the hub frame \\
\hline$\Omega_{h u b}^{\text {eng }}$ & $1 \times 1$ & $\mathrm{rad} \cdot \mathrm{s}^{-1}$ & $\begin{array}{l}\text { Hub angular velocity during the engage } \\
\text { operation }\end{array}$ \\
\hline$\Omega_{h u b}^{d i s}$ & $1 \times 1$ & $\operatorname{rad} \cdot \mathrm{s}^{-1}$ & $\begin{array}{l}\text { Hub angular velocity during the disen- } \\
\text { gage operation }\end{array}$ \\
\hline$t_{\text {eng }}$ & $1 \times 1$ & $\mathbf{s}$ & Engagement time \\
\hline
\end{tabular}


$t_{\text {eng }} \quad 1 \times 1$

s

Disengagement time

\section{Chapter 4}

\begin{tabular}{|c|c|c|c|}
\hline Symbol & Size & SI Unit & Definition \\
\hline$D$ & --- & --- & $\begin{array}{l}\text { Domain of a nonlinear dynamical sys- } \\
\text { tem }\end{array}$ \\
\hline$R^{n}$ & $n$ & - - & Range of a nonlinear dynamical system \\
\hline$L$ & $1 \times 1$ & -- & Lipschitz constant \\
\hline$x^{e}$ & -- & -- & $\begin{array}{l}\text { Equilibrium point of nonlinear dynam- } \\
\text { ical system }\end{array}$ \\
\hline$V(x)$ & $1 \times 1$ & --- & $\begin{array}{l}\text { Lyapunov function of an autonomous } \\
\text { dynamical system }\end{array}$ \\
\hline$V$ & $\begin{array}{l}1 \times 1 \\
\therefore\end{array}$ & -- & $\begin{array}{l}\text { Lyapunov function of an autonomous } \\
\text { dynamical system }\end{array}$ \\
\hline$W(x, t)$ & $1 \times 1$ & --- & $\begin{array}{l}\text { Lyapunov function of a non- } \\
\text { autonomous dynamical system }\end{array}$ \\
\hline$T$ & $1 \times 1$ & $\mathrm{~kg} \cdot \mathrm{m}^{2} \cdot \mathrm{s}^{-2}$ & Kinetic energy \\
\hline$U^{s t}$ & $1 \times 1$ & $\mathrm{~kg} \cdot \mathrm{m}^{2} \cdot \mathrm{s}^{-2}$ & Strain energy \\
\hline$P^{d a m}$ & $1 \times 1$ & $\mathrm{~kg} \cdot \mathrm{m}^{2} \cdot \mathrm{s}^{-3}$ & Work done by damping loads \\
\hline Paer & $1 \times 1$ & $\mathrm{~kg} \cdot \mathrm{m}^{2} \cdot \mathrm{s}^{-3}$ & Work done by aerodynamic loads \\
\hline
\end{tabular}




\begin{tabular}{|c|c|c|c|}
\hline$P^{g r}$ & $1 \times 1$ & $\mathrm{~kg} \cdot \mathrm{m}^{2} \cdot \mathrm{s}^{-3}$ & Work done by gravity loads \\
\hline$P^{a c t}$ & $1 \times 1$ & $\mathrm{~kg} \cdot \mathrm{m}^{2} \cdot \mathrm{s}^{-3}$ & $\begin{array}{l}\text { Power induced in the blade through ac- } \\
\text { tuation loads }\end{array}$ \\
\hline$P^{a w}$ & $1 \times 1$ & $\mathrm{~kg} \cdot \mathrm{m}^{2} \cdot \mathrm{s}^{-3}$ & Actuation power loss \\
\hline$P^{\text {bou }}$ & $1 \times 1$ & $\mathrm{~kg} \cdot \mathrm{m}^{2} \cdot \mathrm{s}^{-3}$ & $\begin{array}{l}\text { Power induced in the blade through its } \\
\text { boundaries }\end{array}$ \\
\hline$L$ & $1 \times 1$ & $\mathrm{~m}$ & Blade length \\
\hline$X$ & $24 \times 1$ & --- & Actual state vector \\
\hline$X^{d}$ & $24 \times 1$ & --- & Desired state vector \\
\hline$X^{*}$ & $24 \times 1$ & --- & Deviated state vector \\
\hline$T^{d}$ & $1 \times 1$ & $\mathrm{~kg} \cdot \mathrm{m}^{2} \cdot \mathrm{s}^{-2}$ & Desired kinetic energy \\
\hline$T^{*}$ & $1 \times 1$ & $\mathrm{~kg} \cdot \mathrm{m}^{2} \cdot \mathrm{s}^{-2}$ & Deviated kinetic energy \\
\hline$U^{s t^{d}}$ & $1 \times 1$ & $\mathrm{~kg} \cdot \mathrm{m}^{2} \cdot \mathrm{s}^{-2}$ & Desired strain energy \\
\hline$U^{s t^{*}}$ & $1 \times 1$ & $\mathrm{~kg} \cdot \mathrm{m}^{2} \cdot \mathrm{s}^{-2}$ & Deviated strain energy \\
\hline$C^{b a}$ & $3 \times 3$ & --- & $\begin{array}{l}\text { Rotation matrix from untwisted, un- } \\
\text { curved, undeformed reference frame to } \\
\text { twisted, curved, undeformed frame }\end{array}$ \\
\hline$C^{B a}$ & $3 \times 3$ & --- & $\begin{array}{l}\text { Rotation matrix from untwisted, un- } \\
\text { curved, undeformed frame to twisted, } \\
\text { curved, deformed frame }\end{array}$ \\
\hline
\end{tabular}




\begin{tabular}{|c|c|c|c|}
\hline$U$ & $3 \times 1$ & $\mathrm{~m}$ & $\begin{array}{l}\text { Displacement of the twisted, curved, } \\
\text { deformed frame with respect to } \\
\text { twisted, curved, undeformed frame }\end{array}$ \\
\hline$\Delta x_{n}$ & $1 \times 1$ & $\mathbf{m}$ & Discretized element length \\
\hline$P^{d a m^{*}}$ & $1 \times 1$ & $\mathrm{~kg} \cdot \mathrm{m}^{2} \cdot \mathrm{s}^{-3}$ & Deviated work done by damping loads \\
\hline$P^{a e r *}$ & $1 \times 1$ & $\mathrm{~kg} \cdot \mathrm{m}^{2} \cdot \mathrm{s}^{-3}$ & $\begin{array}{l}\text { Deviated work done by aerodynamic } \\
\text { loads }\end{array}$ \\
\hline$P^{g^{*}}$ & $1 \times 1$ & $\mathrm{~kg} \cdot \mathrm{m}^{2} \cdot \mathrm{s}^{-3}$ & Deviated work done by gravity loads \\
\hline$P^{\text {act* }}$ & $1 \times 1$ & $\mathrm{~kg} \cdot \mathrm{m}^{2} \cdot \mathrm{s}^{-3}$ & $\begin{array}{l}\text { Deviated power induced in the blade } \\
\text { through actuation loads }\end{array}$ \\
\hline$P^{a w^{*}}$ & $1 \times 1$ & $\mathrm{~kg} \cdot \mathrm{m}^{2} \cdot \mathrm{s}^{-3}$ & Deviated actuation power loss \\
\hline$P^{b o u^{*}}$ & $1 \times 1$ & $\mathrm{~kg} \cdot \mathrm{m}^{2} \cdot \mathrm{s}^{-3}$ & $\begin{array}{l}\text { Deviated power induced in the blade } \\
\text { through its boundaries }\end{array}$ \\
\hline$\beta$ & $1 \times 1$ & --- & Continuous control design factor \\
\hline$\beta_{i}$ & $1 \times 1$ & --- & $\begin{array}{l}\text { Control design factor in the } i^{\text {th }} \text { dis- } \\
\text { cretized element }\end{array}$ \\
\hline$v$ & $1 \times 1$ & $\mathrm{~V} \cdot \mathrm{s}^{-1} \cdot \mathrm{m}^{-1}$ & $\begin{array}{l}\text { Time rate of change of electric field } \\
\text { across active fibres }\end{array}$ \\
\hline $\bar{v}_{i}$ & $1 \times 1$ & $\mathrm{~V} \cdot \mathrm{s}^{-1} \cdot \mathrm{m}^{-1}$ & $\begin{array}{l}\text { Average of } v \text { in the } i^{\text {th }} \text { discretized ele- } \\
\text { ment }\end{array}$ \\
\hline
\end{tabular}


Chapter 5

\begin{tabular}{|c|c|c|c|}
\hline Symbol & Size & SI Unit & Definition \\
\hline$n c$ & $1 \times 1$ & --- & Number of comparison functions \\
\hline$\Phi_{i}(x)$ & $1 \times 1$ & --- & Typical comparison function \\
\hline$\Xi_{l}(x)$ & $1 \times 1$ & --- & Typical weighting function \\
\hline$q_{i}(t)$ & $24 \times 1$ & --- & Generalized temporal function \\
\hline$\left|q_{i}(t)\right|$ & $24 \times 1$ & $-\cdots$ & $\begin{array}{l}\text { Magnitude of generalized temporal } \\
\text { function }\end{array}$ \\
\hline$\Phi_{i}^{\mathrm{O}}$ & $3 \times 24$ & --- & Comparison function of $O$ \\
\hline$P_{l}(x / L)$ & $1 \times 1$ & $--\rightarrow$ & $\begin{array}{l}\text { The } l^{\text {th }} \text { normalized shifted Legendre } \\
\text { polynomial }\end{array}$ \\
\hline$A_{l i}$ & $24 \times 24$ & $\mathrm{~kg} \cdot \mathrm{m}^{2} \cdot \mathrm{s}^{-2}$ & Two-dimensional coefficient tensor \\
\hline$B_{l i}$ & $24 \times 24$ & $\mathrm{~kg} \cdot \mathrm{m}^{2} \cdot \mathrm{s}^{-3}$ & Two-dimensional coefficient tensor \\
\hline$C_{l i j}$ & $24 \times 24 \times 24$ & $\mathrm{~kg} \cdot \mathrm{m}^{2} \cdot \mathrm{s}^{-4}$ & Three-dimensional coefficient tensor \\
\hline$E_{l i / 3}$ & $24 \times 8$ & $\mathrm{~kg} \cdot \mathrm{m}^{2} \cdot \mathrm{s}^{-3}$ & Two-dimensional coefficient tensor \\
\hline$F_{l}$ & $24 \times 1$ & $\mathrm{~kg} \cdot \mathrm{m}^{2} \cdot \mathrm{s}^{-3}$ & One-dimensional coefficient tensor \\
\hline$I$ & $3 \times 3$ & --- & Identity matrix \\
\hline$\Psi_{i}^{\mathrm{O}}$ & $3 \times 24$ & --- & Constant coefficient matrix in $\Phi_{i}^{\bigcirc}$ \\
\hline$\Psi_{i}^{U}$ & $3 \times 3$ & --- & Constant coefficient matrix in $\Phi_{i}^{U}$ \\
\hline
\end{tabular}




\begin{tabular}{|c|c|c|c|}
\hline$\Psi_{i}^{C B b}$ & $3 \times 3$ & --- & Constant coefficient matrix in $\Phi_{i}^{C^{B b}}$ \\
\hline$\delta_{i-1}$ & $1 \times 1$ & --- & Kronecker delta function \\
\hline$U_{i}(t)$ & $3 \times 1$ & -- & Generalized displacement \\
\hline$U_{i}$ & $3 \times 1$ & --- & Generalized displacement \\
\hline$C^{B b}(t)$ & $3 \times 3$ & --- & Generalized rotation matrix \\
\hline$C^{B b}$ & $3 \times 3$ & --- & Generalized rotation matrix \\
\hline$H_{i i}$ & $3 \times 3$ & --- & Two-dimensional coefficient tensor \\
\hline$\Delta_{i}$ & $3 \times 1$ & -- & One-dimensional coefficient tensor \\
\hline$K_{i}$ & $3 \times 1$ & -- & One-dimensional coefficient tensor \\
\hline$N_{i j}$ & $3 \times 24$ & -- & Two-dimensional coefficient tensor \\
\hline$X_{i i}$ & $3 \times 3$ & -- & Two-dimensional coefficient tensor \\
\hline$Y_{i i}$ & $3 \times 3$ & --- & Two-dimensional coefficient tensor \\
\hline$Z_{j i}$ & $24 \times 3$ & --- & Two-dimensional coefficient tensor \\
\hline
\end{tabular}

\section{Operator Operation}

$\bigcirc^{\prime} \quad$ Derivative of $\bigcirc$ with respect to the blade beam reference line $x$

$\dot{\bigcirc} \quad$ Derivative of $\bigcirc$ with respect to time $t$

|OI Magnitude or absolute value of $\mathrm{O}$ 
$\widetilde{O}$ Skew-symmetric operator that transforms the vector $\{\bigcirc\}$ to the matrix [O]

$\int_{0}^{L} \bigcirc \quad$ Definite integral of $\bigcirc$ with respect to the blade beam reference line $x$

$\bigcirc^{T} \quad$ Transpose of $\bigcirc$

! Factorial operator

\begin{tabular}{cllll} 
Chapter & Index & From & To & Description \\
\hline \hline 2 & $n$ & 0 & $M$ & Generalized airfoil motion number \\
2 & $n$ & 1 & $N$ & Inflow state number \\
5 & $l, i, j$ & 1 & $n c$ & Comparison function number \\
\hline
\end{tabular}




\section{Chapter 1}

\section{Introduction}

Since their invention, given their capability to hover as well as perform vertical takeoff and landing, helicopters have been able to perform operations that are beyond any other air vehicle's capability. Maritime operations such as search and rescue, short-distance transportation, close air inspection and boarding, and vertical replenishments can be done exclusively by helicopters.

To operate at sea, flight decks can be either fixed (oil rigs) or moving (ships). Oscillatory motion of these flight decks added to the hostile environment of the sea, where two different fluids (air and water) interact with a fixed or a moving structure, present significant challenges to the aircraft and the ship crew. These challenges, which are either operational or safety-related, mostly occur during the take-off and landing phases of flight in which there are significant Dynamic Interface (DI) problems between the helicopter and the flight deck (ship). Among DI problems is the Blade Sailing Phenomenon (BSP) that its modelling and control is the subject of this thesis. 


\subsection{Blade Sailing Phenomenon Definition}

Blade sailing is a transient aeroelastic phenomenon described by the large undesired flapping motion of the helicopter rotor blades during low rotor speeds (small centrifugal stiffening), under high wind, and elevated sea conditions. Large flapping motion can result in blade contact with the aircraft tailboom or tunnel in the case of tandem helicopters, deck crew and in extreme cases the flight deck. Contact with the aircraft is termed tunnel/tailboom strikes [1].

The major cause of vibratory flapping motion of blades is that blades are flexible beam-like structures that at low centrifugal stiffening can experience large nonlinear flapwise bending deformations when subjected to dynamic loads or moving supports.

Dynamic loads on the blade are mostly aerodynamic forces and moments that depend on the airflow properties around the blade and its structural properties. Complex airflow distribution around the blade is an outcome of the interaction between many different factors including the relative angle between the free stream flow and the ship deck, the ship geometry, and deck structures. This airflow causes significant rapid changes in the aerodynamic loads while the blades are rotating. Due to low structural stiffness of the blades in flapwise bending, they can experience large deformations in response to the external loads such as aerodynamic forces and moments. These deformations, in an aeroelastic cycle of cause and effect, change the flow distribution on the blade and as a result affect the aerodynamic loads acting on them.

The rotor articulation mechanism transfers the oscillatory motion of the flight deck to the blades through the helicopter body. This motion can also cause significant deformation of the blades even at the absence of the aerodynamic loads because it induces some energy in the blades through its boundary interaction (the blade root).

Flapwise deformations of rotor blades in the absence of any rotation can easily be 
restricted by securing and tieing down the blades. However, during start-up and shutdown phases, these deformations cannot be controlled using external devices such as cables. Excessive blade deformations along with a number of other operational restrictions have resulted in establishing a set of Ship Helicopter Operational Limits (SHOLs) for safe maritime operations. These limits are determined in terms of the helicopter weight, wind and sea conditions, and the ship motions, through research and flight trials [2].

SHOLs restrict the operational availability of helicopters for many emergency missions that require timely response in harsh weather conditions. Therefore, expanding the SHOLs or even removing these limits using new technologies and operation strategies are definitely worth investigating.

\subsection{Literature Review}

Modelling and control of the BSP is a challenging multi-disciplinary problem and its review can address current advances in the areas of aerodynamics, multibody dynamics, and active control. Any of these areas individually is a large research field that completely outlining all developments in the field is beyond the scope of this review. In addition, comprehensive reviews of experimental and analytical investigations into understanding and modelling of the BSP have been reported in the literature $[3,4]$. These reviews cover all factors contributing to this phenomenon. Therefore, in the following, only relevant literature that pertains directly to compatible aerodynamic load modelling for the BSP control and the control of the BSP are presented.

\subsubsection{Aerodynamics}

The importance of using a powerful aerodynamic model to predict the exact aerodynamic loads on a rotating blade during engagement and disengagement operations 
has been stressed in many BSP investigations [1,3-6]. Among analytical aerodynamic models for aeroelastic phenomena analysis, two models have received more attention from helicopter aerodynamic researchers. An indicial model named Arbitrary Motion Theorem (AMT) and the Unified Airloads Model (UAM).

Both AMT and UAM can predict the unsteady loads in the time domain, allow for arbitrary airfoil motions, and are augmented with an appropriate dynamic stall model. The AMT, which was developed by J. Leishman and his colleagues [7], is originally a frequency domain method that is based on indicial functions and Duhamel's integrals. It is coupled with Leishman-Beddoes (L-B) semi-empirical dynamic stall model [8]. UAM was developed by D. Peters and his colleagues [9-14] and is based on the potential flow assumption that covers arbitrary airfoil deformations as well as its rigid-body motions. This model utilizes the ONERA dynamic stall model.

Earlier aerodynamic models for the BSP investigations utilized a quasi-steady thin airfoil theory augmented with Kirchoff's trailing edge static stall model [15]. Keller $[6,16]$ used nonlinear quasi-steady blade element theory to model the aerodynamic loads for a wide range of angles of attack. He also examined the unsteady aerodynamic effects and concluded that the quasi-steady model was accurate enough to predict the BSP loads.

In a framework of nonlinear finite element methods, where the element library included both rigid and deformable bodies, Bottasso and Bachau developed an aeroelastic program to model the rigid body and the structural deformations of blades related to the BSP $[17,18]$. In their model, they used Peter's unsteady two-dimensional thin airfoil model [10] to calculate the aerodynamic loads and claimed that their finite element based aeroelastic software DYMORE could handle complex aerodynamic/structural configurations.

Most recently, the effectiveness of the quasi-steady method and AMT in predicting the aerodynamic loads on the blade has been tested and verified against experimental 
data $[3,4]$. It was shown that the nonlinear quasi-steady aerodynamic model combined with a dynamic stall model that is modified for Mach number is sufficient for the BSP modelling at low wind speeds. However, at higher wind speeds the AMT was proved to be a more accurate predictor of aerodynamic loads.

\subsubsection{BSP Control and Modelling for Control}

As pointed out in Refs. $[3,4]$, a significant number of systematic investigations have been carried out in order to analyze and understand the BSP. However, there has been a limited number of investigations in applying control techniques to attenuate the BSP during engage/disengage operations. Early investigation in this area goes back to the work done by Keller [6], in which the effectiveness of three passive control strategies and one active feedback control strategy to control the BSP on the H-46 helicopter rotor system were examined.

The first strategy proposed a new procedure to be followed by pilots during engage/disengage operations without any physical changes to the helicopter. In this strategy, to increase the blade flapwise bending stiffness it was suggested that pilots do the engage/disengage operations at higher collective pitches of the blades. Although, this strategy increased the flapwise stiffness, it increased the aerodynamic loads on the blade, and as a result also increased the blade tip deflections in most cases. This deficiency made this strategy ineffective.

The second strategy examined the performance of a discrete flap damper on the blade response during engagement and disengagement. Due to the small feasible stroke of the flap damper, the flap deflections were not reduced in many situations.

The third strategy considered adding the extendible/retractable leading-edge spoilers to the tip of the blade, to reduce its lift and increase its drag and as a result reduce the blade flapping. This strategy was found to be more successful in

decreasing the blade upward and downward flapping deflections. However, the main 
drawback of this strategy was the structural modifications needed on the blade in order to add spoilers and hence, increasing the complexity of the blade system.

The last strategy considered the active control of the helicopter swashplate to attenuate the BSP. Due to the difficulty in designing an active controller based on a nonlinear flexible blade model with nonlinear unsteady aerodynamics, a simple linear quasi-steady aerodynamic model was assumed. Then an optimal controller, based on a linear time invariant flapping model of a rigid blade on a gimballed rotor, was designed. The main deficiency of this strategy is its inability to consider the elastic deformation of the blade while designing the controller. Moreover, the controller was designed and tested on a oversimplified linear model of the blade system.

Ref. [19] has considered the utilization of the trailing edge flap for the reduction of the BSP deflections. It was found that significant blade tip deflection and bending moment reduction can be obtained if the flap is operated in opposition to the blade tip deflection velocity. It was also determined that the farther the flap is from the the blade root and the larger the flap deflection that is allowed, the more control effectiveness is achievable. In addition to the complex mechanism of the flaps on the blade, the fact that renders the reliability of the proposed strategy to be questionable is that this strategy has shown its success only for the stiffer blades, while many current helicopter blades are extremely flexible.

In Ref. [3], a feed-forward control strategy based on the Integral Active Twist (IAT) concept to counter the BSP was proposed and assessed. In this reference, by embedding active piezo fibres in a scaled rotor blade, the blade twist was morphed actively as a function of the wind and the hub angular velocity profile. The results showed considerable reductions mainly in downward deflections of the blade. The main disadvantage of the proposed feed-forward strategy, which is true for all feedforward control strategies, is the high sensitivity of the efficiency of the strategy to the exact measurement of the input disturbances (wind properties in this case). In 
addition, the proposed strategy is highly dependent on finding a critical angular velocity of the blade and associated critical time when the controller turns on. These parameters vary as a function of the blade structural and inertial properties as well as wind speed. These problems render the proposed controller almost infeasible in terms of practical implementation.

Most recently, the effectiveness of two semi-active control strategies involving shape memory alloy (SMA) and magnetorheological damper (MRD) technologies were tested on the BSP [20]. Increasing the stiffness/damping of the flapping motion, it was shown that controllers are successful at tunnel-strike suppression and the reduction of the blade upward tip deflections with unsteady flow conditions over the blade. In this research, after many simplifications, the blade was considered mainly as a rigid body in the flapping motion plane with some modifications to include its bending modes when it hits the droop stops. In addition, the dynamic model on which the controllers were designed did not consider the ship motion and the performance of the proposed controllers were not tested for disengage operations where significant downward tip deflection is expected.

In similar research conducted by the same authors, the performance of a model predictive controller (MPC) on the BSP in the presence of unsteady flow effects was assessed [21]. Without including any physical actuation devices, it was assumed the blade pitch can be used as control commands. Using a number of assumptions to restrict the blade motion to small flapping angles, and considering only the rigidbody motion of the blade, the blade flapping dynamics was modelled as a nonlinear simple state-space system with two states. The results showed that the proposed MPC technique was able to compensate the high lift conditions during engagement by modifying the flapping response of the blade.

Refs. [22,23] proposed a Proportional-Integral-Derivative (PID) Individual Blade 
Root Controller (IBRC) to suppress the tunnel strikes for articulated rotors, considering steady flow conditions during engagement operations. The flapping dynamics of the model were composed of a forced parametric oscillator with nonlinear stiffness and time-varying coefficients. The simulation results showed $50 \%$ reduction in upward deflections and $35 \%$ reduction in downward deflections for a wind velocity of 45 knots. The aerodynamic flapping moment was derived using the blade-element theory with reverse-flow effects. The proposed strategy was not verified against any experimental data with different wind velocity over the deck and was not tested with disengagement operations.

\subsection{Motivation and Objective}

Review of the BSP literature reveals that there is a gap regarding modelling the BSP for control purposes and controlling the structural deformations of the blade during blade sailing. Current BSP models are either too complicated to be the basis for control design, or too simple to model the accurate behaviour of the controlled blade during engagement and disengagement.

Many current BSP control strategies utilize either passive methods or feed-forward active methods. Passive methods refer to all efforts to optimize the shape and therefore the structural properties of the blade or to modify the design of the rotor system. Feed-forward active methods rely on predicted information about the significant factors contributing to the BSP such as ship airwake. These methods then try to change the external (aerodynamic) loads distribution on the blade and then its structural properties during the rotor engage and disengage operations, using some physical control surfaces (such as flaps) or morphing the blade structure. Neither approach uses feedback of the response characteristics of the blade. In an individual blade feedback control system for the BSP, this feedback is used to determine the required 
corrective measure and then to send the corrective commands to actuators to morph the blade. Actuators can be either physical control surfaces (flaps) or integrated smart materials embedded in the blade.

It is well established in control theory and its application that closed-loop feedback control offers the potential for better results than feed-forward or passive control methods in terms of the performance and stability of the controlled system. This superiority becomes more clear when the external loads or disturbances on a system cannot be measured.

As discussed above, all current BSP feedback controllers are designed and tested on simple dynamic and aerodynamic models of the blade that overlook or underestimate the elastic deflections of the blade. Since these controls have never been tested on more exact models of the BSP or on experimental blades, their efficiency cannot be assessed fairly. Therefore, a feedback BSP controller that is designed based on a more exact model of the BSP and is validated against experimental data is clearly required.

To design a feedback controller, one needs to define the dynamics of the model by a set of analytical ordinary differential equations (ODEs), arranged in a statespace form. For feedback control of the BSP, the governing equations are composed of structural dynamics, ship motion dynamics, aerodynamics, and control equations. As such, the dynamics is required to be presented in a compact state-space form. This requirement has been the main motivation to apply the Unified Airloads Model for obtaining the aerodynamic loads on the blade, and to use the Geometrically Exact Intrinsic Beam Model for modelling the nonlinear structural deflections of the blade.

In addition to the analytical state-space form of the aerodynamic equations, the Unified Airloads Model is able to calculate the aerodynamic loads on an active blade that utilizes rigid control surfaces (flaps), as well as various morphing controls such as active twist control. This capability would give the controller designer more options 
to choose the most effective control strategy and to design an efficient BSP control, which may be of interest for future research.

The Intrinsic Beam Model has been previously used by another member of the Applied Dynamics Group (ADG) at Carleton University and has proved its capability in modelling the BSP nonlinear structural deflections [3]. This model was originally developed to analyze anisotropic, non-uniform composite helicopter rotor blades [24]. In addition, intrinsic equations are compact, and analytical, and can be easily transformed to the state-space form using various discretization techniques. Moreover, external loads such as aerodynamic forces and moments can be easily incorporated into the intrinsic equations. These properties have made it an ideal structural model for the BSP control investigation.

Since the structural dynamic equations are in the form of partial differential equations, an appropriate discretization method is required to reduce them to ordinary differential equations suitable for control purposes. In this regard, the Galerkin Spectral Discretization Method that results in a compact set of ordinary differential equations with user-defined required accuracy is employed herein. To the best of the author's knowledge, this research is the first application of the Galerkin Spectral Method to discretization of a blade with varying rotor speed. In Ref. [25], this method was used for vibration control of a rotating blade with constant rotor speed. In that research, a quasi-steady aerodynamic model was used to predict the aerodynamic loads on the blade. In addition, the gravity effects were neglected, assuming smaller contributions to the loads on the blade compared to the aerodynamics. In terms of the modelling, this research can be considered as an improvement to the model developed in Ref. [25]. The new model includes the gravity load, that has significant effect in the low rotor speed BSP. It also considers the unsteady aerodynamic and wind loads that are shown to be important in the BSP analyses [3]. 


\subsection{Overview of the Present Research}

In the following chapters, first the aerodynamic loads acting on a rotating helicopter blade during engage and disengage operations are obtained using the Unified Airloads Model. Then, the structural dynamics of the blade, including structural damping, is modelled applying the Intrinsic Beam Model. Actuation; gravity, and wind load effects are added to the model and the resulting complete governing equations are expressed in terms of the velocity and force variables. In Chapter 4 , a nonlinear feedback controller based on Lyapunov stability theorems is designed. The energy based Lyapunov function is obtained by satisfying the law of conservation of energy in the blade. The designed controller is incorporated in the model in Chapter 5 and the closed-loop equations are discretized through conventional Galerkin Spectral Discretization methods. Finally, Chapters 6 and 7 are dedicated to the simulation results and conclusion of the research, respectively. 


\section{Chapter 2}

\section{Aerodynamic Loads Modelling}

As pointed out in the previous chapter, to design a feedback controller, one needs to define the dynamics of the system by a compact set of analytical ordinary differential equations in a state-space form. For active control of the BSP, the main external loads are the aerodynamic forces and moments on the blade that are required to be presented in the state-space form. This requirement has been the main motivation to apply the Unified Airloads Model [10-13] to model the aerodynamic loads on the blade for BSP control purposes. In addition to the analytical state-space form of the equations, this model is able to predict the aerodynamic loads on an active blade that utilizes rigid control surfaces (flaps), as well as various morphing-based actuations such as active twist. This capability increases the overall controllability of the BSP and gives the control designer a variety of options from which to choose the most effective control strategy and to design an efficient control law to counter the BSP.

\subsection{Unified Airloads Model}

The main properties of the Unified Airloads Model are as follows [12]:

- It is based on a 2D potential flow, with the non-penetration boundary condition 
applied on the airfoil and the Kutta condition applied at the airfoil trailing edge;

- It allows for large translational and rotational motions of any arbitrary reference frame with no small-angle assumptions;

- It supports arbitrary but small elastic deformations of the airfoil within the frame;

- It can be easily coupled with any induced flow model, $2 \mathrm{D}$ or $3 \mathrm{D}$, since the airloads computation is decoupled from the induced flow computation;

- It may be reduced to the classical unsteady aerodynamic theories such Wagner, Theodorsen, Garrick, Isaacs, Greenberg, and Loewy; and

- It provides the aerodynamic loads in a compact matrix form. Therefore, it can be easily incorporated into any sets of state-space dynamic equations.

Unified Airloads Model is composed of four main parts:

- Theoretical unstalled generalized loads;

- Induced flow;

- Static corrections; and

- Dynamic stall.

In the following sections, these steps are briefly discussed and their application to the BSP problem is presented.

\subsubsection{Unstalled Generalized Loads}

Fig. 2.1 shows the reference frame of a 2-D potential flow system. In this figure, $b$ is the airfoil semi-chord, $h(x, t)$ is the deformation of the airfoil in the airfoil reference 


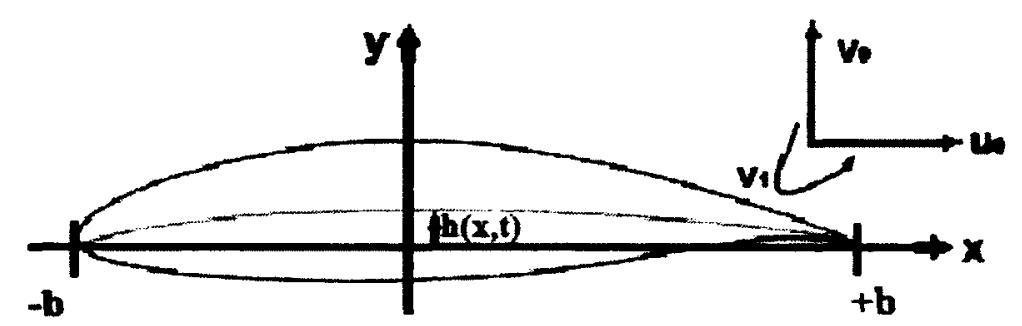

Figure 2.1: Airfoil reference frame.

frame, $u_{0}$ is the velocity of the air in the positive $x$ direction, $v_{0}$ is the velocity of the air in the positive $y$ direction, and $v_{1}$ is the velocity gradient such that the vertical velocity along axis $x$ is given by $v_{0}+v_{1} x / b$.

Assuming small airfoil deformations such that $h<<b, \frac{\partial h}{\partial x}<<1$, and $\frac{\partial h}{\partial t}<<$ $u_{0}$, and using Glauert expansions of the variables $h(x), u_{0}(x), v_{0}(x)$, and $v_{1}(x)$, the unstalled generalized loads can be expressed as follows [12]:

$$
\begin{aligned}
\frac{1}{2 \pi \rho}\left\{L_{n}\right\}= & -b^{2}[M]\left\{\ddot{h}_{n}+\dot{v}_{n}\right\}-b u_{0}[C]\left\{\dot{h}_{n}+v_{n}-\lambda_{0}\right\} \\
& -u_{0}^{2}[k]\left\{h_{n}\right\}-b[G]\left\{\dot{u}_{0} h_{n}+\bar{u}_{0} \zeta_{n}-u_{0} v_{n}+u_{0} \lambda_{0}\right\} \\
\frac{1}{2 \pi \rho} D= & -b\left\{\dot{h}_{n}+v_{n}-\lambda_{0}\right\}[S]\left\{\dot{h}_{n}+v_{n}-\lambda_{0}\right\} \\
& +b\left\{\ddot{h}_{n}+\dot{v}_{n}\right\}^{T}[G]\left\{h_{n}\right\}-u_{0}\left\{\dot{h}_{n}+v_{n}-\lambda_{0}\right\}^{T}[K-H]\left\{h_{n}\right\} \\
& +\left\{\dot{u}_{0} h_{n}+\bar{u}_{0} \zeta_{n}-u_{0} v_{n}+u_{0} \lambda_{0}\right\} \\
\frac{1}{2 \pi} \Gamma= & b\{1\}^{T}[C-G]\left\{\dot{h}_{n}+v_{n}-\lambda_{1}\right\}+u_{0}\{1\}^{T}[K]\left\{h_{n}\right\}
\end{aligned}
$$

In these equations $[K]$ is called stiffness matrix, $[C]$ is the damping matrix, $[G]$ is Greenberg matrix, $[S]$ is the drag matrix, $[M]$ is the mass matrix, and $[H]$ is a coefficient matrix. These matrices together with vectors $\left\{h_{n}\right\},\left\{v_{n}\right\},\left\{\lambda_{0}\right\},\left\{\lambda_{1}\right\}$, and $\{1\}$ are all defined in Appendix A and Ref. [12]. In addition,

- $h_{n}$ is the $n^{\text {th }}$ component of the generalized airfoil motions such that $h_{0}$ represents a rigid-body plunging motion, $h_{1}$ is a rigid-body pitch motion, $h_{2}$ is the airfoil 
camber change, and so on.

- $L_{n}$ is the $n^{\text {th }}$ component of the generalized forces associated with the generalized motions such that $L_{0}$ is the negative lift of the airfoil, $L_{1} b$ is the airfoil nose-up pitching moment about the mid-chord, and so on.

- $\lambda_{0}$ and $\lambda_{1}$ are the first (uniform flow) and the second (flow gradient) coefficients of the induced flow at the airfoil surface, respectively, representing inflow states. They will be obtained from an inflow model that will be discussed in the following sections.

- $\rho$ is the air density.

- $D$ is the airfoil induced drag.

- $\Gamma$ is the total bound circulation on the airfoil.

- $\zeta_{n}$ is the induced velocity at the airfoil produced from some bound circulation other than the airfoil itself.

- $\overline{u_{0}}$ is the velocity of the bound circulation that produces $\zeta_{n}$ with respect to the air mass in the negative $x$ direction.

It should be noted that to include the wind effect, one can easily add the horizontal and vertical components of the wind velocity to the $u_{0}$ and $\lambda_{0}$ terms in the above equations, respectively.

\subsubsection{Induced Flow}

The second part of the Unified Airloads Model is to determine the $\lambda_{0}$ and $\lambda_{1}$ components of the induced flow. Depending on the requirements, various models could be used to estimate the induced flow. For a helicopter in forward flight, one can employ 
the Peters-He 3D dynamic inflow model [10]. However, since for the BSP analysis a 2D aerodynamic model of the rotor blade gives sufficient accuracy [3], in this research a 2D dynamic inflow model is employed. Based on this 2D dynamic inflow model, the ordinary differential equation for the generalized inflow states $\lambda_{n}$ is given by [11]:

$$
\begin{aligned}
& b \dot{\lambda}_{0}-b / 2 \dot{\lambda}_{2}+u_{0} \lambda_{1}=\frac{\dot{\Gamma}}{\pi}+\frac{\dot{\Gamma}_{d s_{0}}}{\pi} \\
& (b / 2 n)\left(\dot{\lambda}_{n-1}-\dot{\lambda}_{n+1}\right)+u_{0} \lambda_{n}=\frac{\dot{\Gamma}}{n \pi} \quad \text { for } \mathrm{n} \geq 2
\end{aligned}
$$

where $\lambda_{i}$ s are inflow states, $\Gamma$ is the total bound circulation on the airfoil, and $\Gamma_{d s_{0}}$ is the circulation loss during dynamic stall obtainable from a dynamic stall model.

Eq. 2.4 is a system of $N$ differential equations with $N+1$ unknown inflow coefficients, $\lambda_{0}, \lambda_{1}, \ldots, \lambda_{n}$. Therefore, one additional equation is required to solve for all of the unknowns. It is shown in Ref. [12] that $\lambda_{0}$ in the two-dimensional case can be approximated by the expression:

$$
\lambda_{0} \approx \frac{1}{2} \sum_{n=1}^{N} b_{n} \lambda_{n}
$$

with the constraint $\sum_{n=1}^{N} b_{n}=1$, where $N$ is the number of chosen inflow states and $b_{n}$ can be obtained using the following relations:

$$
\begin{aligned}
b_{n} & =(-1)^{n+1} \frac{(N+n-1) !}{(N-n-1) !(n !)^{2}} \quad n=1,2, \cdots, N-1 \\
b_{N} & =(-1)^{(N+1)}
\end{aligned}
$$

The inflow equation (Eq. 2.4) can be expressed as the following matrix equation:

$$
\begin{aligned}
\frac{b}{u_{0}}\left[A_{\text {inflow }}\right]\{\dot{\lambda}\}+\{\lambda\}= & \left\{c_{\text {inflow }}\right\}\left(\frac{b}{u_{0}}\left\{e_{\text {inflow }}\right\}^{T}\left\{\dot{v}_{n}+\ddot{h}_{n}\right\}+\left\{f_{\text {inflow }}\right\}^{T}\right. \\
& \left.\left(\left\{\dot{h}_{n}\right\}+\frac{\dot{u}_{0}}{u_{0}}\left\{h_{n}\right\}\right)\right)+\frac{1}{\pi u_{0}}\{1\} \Gamma_{d s_{0}}
\end{aligned}
$$


where $\{\lambda\}=\left\{\lambda_{1} ; \lambda_{2} ; \ldots ; \lambda_{N}\right\}$ and the inflow parameters $\left\{c_{\text {inflow }}\right\},\left\{e_{\text {inflow }}\right\},\left\{f_{\text {inflow }}\right\}$, and $\left[A_{\text {inflow }}\right]$ are given in Appendix $\mathrm{A}$.

\subsubsection{Static Corrections}

Since the generalized loads are calculated considering the airfoil as a thin plate, the results are to be correlated with experimental data to consider the thickness and compressibility effects. The aerodynamic coefficients for thin airfoil are:

$$
\begin{aligned}
& c l_{\alpha}=2 \pi, \quad c l_{0}=2 \pi\left(\dot{h_{0}}+0.5 \hat{h_{1}}\right),\left.\quad c m_{\alpha}\right|_{M C}=\frac{\pi}{2} \\
& \left.c m_{\alpha}\right|_{C R}=\frac{\pi}{2}[1+2 a], \quad c m_{0}=-\frac{\pi}{4}\left(\dot{h_{1}}+0.5 \hat{h_{2}}\right)
\end{aligned}
$$

where $c l_{\alpha}$ is the slope of the airfoil lift coefficient curve, $c l_{0}$ is the lift coefficient at zero angle of attack, $\left.\mathrm{cm}_{\alpha}\right|_{M C}$ is the slope of the pitching moment curve at mid-chord, $\left.c m_{\alpha}\right|_{C R}$ is the slope of the pitching moment curve at the center of rotation, $h_{0}^{\prime}$ is the spatial derivative of the airfoil camber along its chord, $h_{1}^{\prime} / b$ is the spatial derivative of the airfoil angle of attack along its chord, $h_{2}^{\prime} / b$ is the spatial derivative of the airfoil curvature along its chord, and $a$ is the distance between the airfoil center of rotation and its mid-chord per unit semi-chord. This $a$ is positive if the center of rotation is aft of mid-chord. Ref. [13] gives a complete set of these parameters along with zero-lift angle of attack $\left(\alpha_{0 L}\right)$ for NACA xx12 airfoils. As expected, in symmetric airfoils such as NACA 0012, $h_{0}^{\prime}, h_{1}^{\prime}$, and $h_{2}^{\prime}$ are equal to zero. Hence, assuming $a=-1 / 2$, the thin airfoil coefficients for symmetric airfoils that are extensively used in helicopter rotor blades are:

$$
c l_{\alpha}=2 \pi, \quad c l_{0}=0,\left.\quad c m_{\alpha}\right|_{M C}=\frac{\pi}{2},\left.\quad c m_{\alpha}\right|_{\alpha=-1 / 2}=0, \quad c m_{0}=0
$$


Since experimental data show different values for different airfoils for the abovementioned aerodynamic parameters, some correction factors are required to obtain the correct unstalled loads on the airfoil. As an example, wind tunnel data for the NACA 0012 airfoil shows that for Reynolds numbers $(R e)$ between $2 \times 10^{6}$ and $2 \times 10^{7}$, the lift curve slope can be approximated in terms of the Mach number $(M)$ by:

$$
c l_{\alpha}=\frac{5.8728+0.2997 \log \left(R e / 10^{6}\right)}{\sqrt{1-M^{2}}}
$$

As a result, the thin airfoil value $c l_{\alpha}=2 \pi$ must be multiplied by $f_{L}=c l_{\alpha} / 2 \pi$ to obtain the correct value for the slope of the lift coefficient curve.

For rigid airfoils with no trailing edge flaps, Ahaus and Peters [13] propose three static correction factors: 1) correction to $c l$ or $f_{L}, 2$ ) correction to $c m$ or $f_{M}$, and 3) correction to the airfoil center of rotation or $a_{C O R}$. It is shown that $f_{M}$, and $a_{C O R}$ are dependent; therefore by choosing $f_{M}=1$ we can correct the airfoil pitching moment coefficient by finding $a_{C O R}$. The corrected lift and moment coefficients are thus:

$$
c l=f_{L}\left(\frac{-L_{0}}{\rho u_{0}^{2} b}\right), \quad c m=f_{M}\left(\frac{L_{1} b-L_{0} a_{C O R} b}{2 \rho u_{0}^{2} b^{2}}\right)
$$

One deficiency of the unified model is its inability to give the profile drag of the airfoil. As such, this kind of drag must be added to the induced drag, provided by the model, to get the total drag of the airfoil. This topic is discussed further in the following sections where application of the unified model to the BSP aerodynamic modelling is presented.

\subsubsection{Dynamic Stall}

Theoretically, when the flow separates from the airfoil surface, and the airfoil loses its lift, stall happens. Dynamic stall can be referred to as the time delay and the 
overshoot that appears in the lift response of the airfoil to a rapid change in its angle of attack. The rapid change causes flow separation over the leading-edge of the airfoil and produces strong vortex shedding from the leading-edge. This vortex that contains high-velocity airflows increases the lift produced by the airfoil. As the rate of change of the angle of attack becomes zero and then a negative value, the vortex detaches from the leading-edge and passes behind the trailing-edge. Then lift reduces significantly and the static stall starts.

So far, a number of dynamic stall models have been developed. Among them, Leishman-Beddoes (L-B) [8] and ONERA [26] models have received more attention by helicopter aeroelasticitists. There are three main reasons that make the ONERA model more preferable over the L-B one from an aeroelastic control point of view:

First, while the ONERA model is developed in state-space form, the L-B method is originally formulated in indicial function form. This formulation makes the $\mathrm{L}-\mathrm{B}$ model not suitable for control applications. Although the state-space formulation of the LB model is available, this formulation is intended for aerolastic applications, such as wind turbines, where using thick airfoils and low RPM rotors, the compressibility effects and the leading-edge flow separation can be neglected [27]. Second, some researchers have reported that for low Mach number flights $(M<0.3)$, the L-B dynamic stall model does not predict the stall behaviour of the blade as well as it does for higher Mach number flights [3]. To the best of author's knowledge, neither of these two problems is reported about ONERA stall model. Finally, the ONERA dynamic stall model can be easily incorporated into any airloads model such as the Unified Airloads Model [14]. Therefore, to meet the main objective of this research, BSP control, which happens at low Mach numbers, the ONERA model is employed here.

As outlined earlier, in the stalled regime, the airloads have a time delay and an overshoot due to the passing of shed vorticity. To take these two charactrisitics into 
account, a second order transfer function with the static stall residual acting as a forcing function is used in the ONERA dynamic stall model. In the Unified Airloads Model, an extended version of the original ONERA model for helicopter applications that allows for unsteady free stream and large angles of attack and plunge motions, is adopted [14].

The extended ONERA dynamic stall model can be expressed as:

$$
\frac{b^{2}}{u_{T}^{2}} \ddot{\Gamma}_{d s_{n}}+\eta_{d s} \frac{b}{u_{T}} \dot{\Gamma}_{d s_{n}}+\omega_{d s}^{2} \Gamma_{d s_{n}}=-b u_{T} \omega_{d s}^{2}\left[\Delta c_{n}+e_{d s} \frac{d \Delta c_{n}}{d t} \frac{b}{u_{T}}\right], \quad n=0,1, \cdots, M+1
$$

where $\Gamma_{d s_{n}}$ is the dynamic stall state corresponding to the $n^{\text {th }}$ generalized load, $u_{T}=\sqrt{u_{0}^{2}+\left(v_{0}+\dot{h}_{0}-\lambda_{0}\right)^{2}}$ is the total air velocity over the airfoil and can be approximated as $u_{T}=u_{0}, \omega_{d s}=\omega_{d s_{0}}+\omega_{d s_{1}}(\Delta c l)^{2}$ is the dynamic stall frequency parameter, $\eta_{d s}=\eta_{d s_{0}}+\eta_{d s_{1}}(\Delta c l)^{2}$ is the dynamic stall damping parameter, $e_{d s}=e_{d s_{1}}(\Delta c l)^{2}$ is the dynamic stall time delay parameter, $\Delta c_{n}$ is the static residual of the $n^{\text {th }}$ generalized load, and $\Delta c l$ is the lift static residual. All these parameters can be obtained from experimental data.

Static stall residuals are the difference between linear quasi-steady thin airfoil values (corrected for the airfoil thickness and the compressibility effect) and the nonlinear static experimental (or CFD) values. In other words, these residuals show the difference between the values predicted by a thin airfoil model that cannot take into account the static stall effect, and the real experimental (CFD) values, that includes the static stall effect. The airfoil lift and moment residuals can be obtained from 
experimental data by [9]:

$$
\begin{aligned}
\Delta c l= & \frac{1}{2}\left(c l_{0}+c l_{1}\right) f \cos \alpha+\frac{1}{2}\left(c l_{0}-c l_{1}\right) \cos \alpha+c l_{\alpha} f \sin \alpha-c l_{\exp } \\
\Delta c m= & \frac{1}{2}\left(c m_{0}+c m_{1}\right) f \cos ^{2} \alpha+\frac{1}{2}\left(c m_{0}-c m_{1}\right) \cos ^{2} \alpha+c l_{\alpha} f \sin \alpha \cos \alpha+ \\
& \frac{a}{2} c d \sin \alpha-c m_{\exp }
\end{aligned}
$$

where $c l_{0}$ and $c l_{1}$ are the lift curve slope at $\alpha=0$ and $\alpha=180$, respectively; $c m_{0}$ and $c m_{1}$ are the moment curve slope at $\alpha=0$ and $\alpha=180$, respectively; and $c l_{\exp }$ and $c m_{\exp }$ are the experimental values of the lift coefficient and the moment coefficient. If one decides not to make static corrections, Eq. 2.9 can be used to obtain the thin airfoil values.

Similarly, the drag residual may be obtained by:

$$
\Delta c d=c d_{e x p}-\left(c d_{0}+c d_{i}\right)
$$

where $c d_{0}$ is the the profile drag coefficient (drag coefficient at $\alpha=0$ ) and $c d_{i}$ is the linear induced drag coefficient obtained from the thin airfoil values.

Finally, the generalized loads, including dynamic stall, may be obtained by:

$$
\begin{aligned}
& L_{T_{0}}=L_{0}-\rho u_{0} \Gamma_{d s_{0}}-\rho b c d_{0} u_{T}\left(v_{0}+\dot{h}_{0}-\lambda_{0}\right) \\
& L_{T_{n}}=L_{n}+\rho u_{T} \Gamma_{d s_{n}}, \quad n \geq 1 \\
& D_{T}=D+\rho b c d_{0} u_{T} u_{0}-\rho\left(v_{0}+\dot{h}_{0}-\lambda_{0}\right) \Gamma_{d s_{0}}
\end{aligned}
$$

where $L_{T_{0}}$ is the negative total lift, $L_{T_{n}}$ is the $n^{\text {th }}$ component of the total generalized loads, $D_{T}$ is the total drag of the airfoil, and $L_{n}$ is the $n^{\text {th }}$ component of the linear generalized loads obtained in part one of the Unified Airloads Model development. 


\subsection{BSP Aerodynamic Loads}

Following the above three steps, the statically-corrected, dynamic-stall-included lift, drag, and pitching moment of a rigid airfoil can be obtained by:

$$
\begin{gathered}
\text { Lift }=\underbrace{-f_{L} L_{0}+\rho b c d_{0} u_{T}\left(v_{0}+\dot{h}_{0}-\lambda_{0}\right)+\rho b c l_{0} u_{T} u_{0}}_{\text {Statically-corrected linear lift }} \underbrace{+\rho u_{0} \Gamma_{d s_{0}}}_{\text {Dynamic stall effect }} \\
\text { Drag }=\underbrace{D+\rho b c d_{0} u_{T} u_{0}-\rho b c l_{0} u_{T}\left(v_{0}+\dot{h}_{0}-\lambda_{0}\right)}_{\text {Statically-corrected drag }} \underbrace{-\rho\left(v_{0}+\dot{h}_{0}-\lambda_{0}\right) \Gamma_{d s_{0}}}_{\text {Dynamic stall effect }} \\
\underbrace{+\left[\left(\rho u_{T} \Gamma_{\text {ds }}\right) b+\left(\rho u_{0} \Gamma_{d s_{0}}\right) a_{C O R} b\right]}_{\text {Dynamic stall effect }}
\end{gathered}
$$

where $u_{T}$ is the total air velocity over the airfoil and $c d_{0}, c l_{0}$, and $c m_{0}$ are the aerodynamic coefficients of the airfoil at zero angle of attack that can be obtained either from Eq. 2.8 or from experimental airfoil data for a static airfoil.

As will be shown in the next chapter, for BSP control, the aerodynamic loads must be defined in the deformed structural frame. Fig. 2.2 shows the deformed structural and the aerodynamic frames of reference, respectively, as the $B$ and $a$ systems, both of which are attached to the airfoil. While the origin of the deformed structural frame is located at the shear center (the airfoil center of rotation that is almost the quarter-chord point on the airfoil), the origin of the aerodynamic frame is placed at the mid-chord point. It can be seen that the aerodynamic loads can be defined in the 


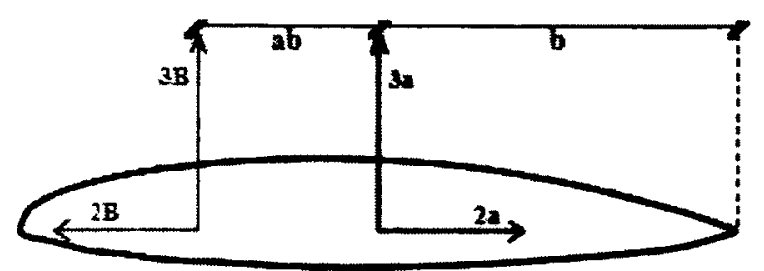

Figure 2.2: Aerodynamic(a) and deformed structural(B) frames.

deformed structural frame as follows:

$$
{ }^{B} f^{a}=\left\{\begin{array}{c}
{ }^{B} f_{1}^{a} \\
{ }^{B} f_{2}^{a} \\
{ }^{B} f_{3}^{a}
\end{array}\right\}=\left\{\begin{array}{c}
0 \\
-D r a g \\
\text { Lift }
\end{array}\right\},{ }^{B} m^{a}=\left\{\begin{array}{c}
{ }^{B} m_{1}^{a} \\
{ }^{B} m_{2}^{a} \\
{ }^{B} m_{3}^{a}
\end{array}\right\}=\left\{\begin{array}{c}
\text { Moment } \\
0 \\
0
\end{array}\right\}
$$

where ${ }^{B} f^{a}$ and ${ }^{B} m^{a}$ denote the aerodynamic forces and moments defined in the deformed structural frame ( $B$ frame), respectively.

Given the fact that the chord and the thickness of a helicopter blade cross section are very small compared to its span, the structural flexibility of the cross section can be ignored. As a result, all $h_{n} \mathrm{~s}$ for $n \geq 2$ and their corresponding generalized loads $\left(L_{n} \mathrm{~s}\right)$ are assumed to be zero. Since the aerodynamic frame is rigidly attached to the airfoil, this frame is subjected to the same linear and angular velocities and accelerations as the blade cross section. Hence, $h_{0}$ and $h_{1}$ that are the plunge and pitching motions of the airfoil with respect to this moving aerodynamic frame are zero. The relationship between the velocities in the aerodynamic and the deformed structural frames can be summarized as follows: 


$$
\begin{aligned}
u_{0} & ={ }^{a} V_{2}={ }^{B} V_{2} \\
-v_{0} & ={ }^{a} V_{3}={ }^{B} V_{3}+a b^{B} \Omega_{1} \\
v_{1} & =b^{B} \Omega_{1}
\end{aligned}
$$

where $\Omega_{1}$ is the angular velocity of the airfoil about its center of rotation, superscript $a$ indicates that the variable is defined in the aerodynamic frame, and superscript $B$ shows that the variable is defined in the deformed structural frame. In this development, the center of rotation is placed at the origin of the structural frame (quarterchord point of the airfoil). Therefore, $a=-1 / 2$. In addition, the static correction factors can be obtained from the following equations:

$$
\begin{gathered}
f_{L} \equiv \frac{\left(\frac{\partial c l}{\partial \alpha}\right)_{\text {exp }}}{\left(\frac{\partial c l}{\partial \alpha}\right)_{t h e}}=\frac{c l_{\alpha}}{2 \pi} \\
f_{M} \equiv \frac{\left(\frac{\partial c m}{\partial \alpha}\right)_{\text {exp }}}{\left(\frac{\partial c m}{\partial \alpha}\right)_{t h e}}=\frac{c m_{\alpha}}{\frac{\pi}{2}\left[1+2 a_{C O R}\right]}=1 \Longrightarrow a_{C O R}=\frac{c m_{\alpha}}{\pi}-\frac{1}{2}
\end{gathered}
$$

Substituting Eqs. 2.23-2.25 in Eqs. 2.19-2.21, and then in Eq. 2.22, and including the wind velocity components $V_{1 w}, V_{2 w}$, and $V_{3 w}$, the aerodynamic loads in the deformed structural frame can be expressed as:

$$
\begin{aligned}
f_{2}^{a}= & c l_{\alpha} \rho b\left(-V_{3}-a b \Omega_{1}-\frac{1}{2} \sum_{n=1}^{N} b_{n} \lambda_{n}-V_{3 w}\right)^{2}- \\
& \rho b c d_{0}\left(\left(V_{2}+V_{2 w}\right)^{2}+\left(-V_{3}-a b \Omega_{1}-\frac{1}{2} \sum_{n=1}^{N} b_{n} \lambda_{n}-V_{3 w}\right)^{2}\right)^{\frac{1}{2}}\left(V_{2}+V_{2 w}\right) \\
& +\rho b c l_{0}\left(\left(V_{2}+V_{2 w}\right)^{2}+\left(-V_{3}-a b \Omega_{1}-\frac{1}{2} \sum_{n=1}^{N} b_{n} \lambda_{n}-V_{3 w}\right)^{2}\right)^{\frac{1}{2}} \\
& \left(-V_{3}-a b \Omega_{1}-\frac{1}{2} \sum_{n=1}^{N} b_{n} \lambda_{n}-V_{3 w}\right)
\end{aligned}
$$




$$
\begin{aligned}
f_{3}^{a}= & c l_{\alpha} \rho b\left(V_{2}+V_{2 w}\right)\left(-V_{3}-a b \Omega_{1}-\frac{1}{2} \sum_{n=1}^{N} b_{n} \lambda_{n}-V_{3 w}\right)+ \\
& \frac{1}{2} c l_{\alpha} \rho b^{2}\left(V_{2}+V_{2 w}\right) \Omega_{1}+\frac{1}{2} c l_{\alpha} \rho b^{2}\left(-\dot{V}_{3}-a b \dot{\Omega}_{1}\right)+ \\
& \rho b c l_{0}\left(\left(V_{2}+V_{2 w}\right)^{2}+\left(-V_{3}-a b \Omega_{1}-\frac{1}{2} \sum_{n=1}^{N} b_{n} \lambda_{n}-V_{3 w}\right)^{2}\right)^{\frac{1}{2}}\left(V_{2}+V_{2 w}\right) \\
& +\rho b c d_{0}\left(\left(V_{2}+V_{2 w}\right)^{2}+\left(-V_{3}-a b \Omega_{1}-\frac{1}{2} \sum_{n=1}^{N} b_{n} \lambda_{n}-V_{3 w}\right)^{2}\right)^{\frac{1}{2}} \\
& \left(-V_{3}-a b \Omega_{1}-\frac{1}{2} \sum_{n=1}^{N} b_{n} \lambda_{n}-V_{3 w}\right)
\end{aligned}
$$

$$
\begin{aligned}
& m_{1}^{a}=-\frac{1}{16} \rho b^{2}\left(-32 c m_{0} V_{2}^{2}-64 c m_{0} V_{2} V_{2 w}-32 c m_{0} V_{2 w}^{2}-32 c m_{0} V_{3}^{2}-\right. \\
& 64 c m_{0} a b V_{3} \Omega_{1}-64 c m_{0} V_{3}\left(\frac{1}{2} \sum_{n=1}^{N} b_{n} \lambda_{n}\right)-64 c m_{0} V_{3} V_{3 w}-32 c m_{0} a^{2} b^{2} \Omega_{1}^{2}- \\
& 64 c m_{0} a b \Omega_{1} \lambda_{0}-64 c m_{0} a b \Omega_{1} V_{3 w}-32 c m_{0}\left(\frac{1}{2} \sum_{n=1}^{N} b_{n} \lambda_{n}\right)^{2}-32 c m_{0} V_{3 w}^{2}- \\
& 64 c m_{0}\left(\frac{1}{2} \sum_{n=1}^{N} b_{n} \lambda_{n}\right) V_{3 w}+8 c l_{\alpha} V_{2}\left(\frac{1}{2} \sum_{n=1}^{N} b_{n} \lambda_{n}\right)+c l_{\alpha} b^{2} \dot{\Omega}_{1}+32 c m_{\alpha} V_{2} V_{3 w} \\
& +8 c l_{\alpha} V_{2} V_{3}+16 c m_{\alpha} b \dot{V}_{3}+32 c m_{\alpha} V_{2}\left(\frac{1}{2} \sum_{n=1}^{N} b_{n} \lambda_{n}\right)+8 c l_{\alpha} V_{2} V_{3 w}+ \\
& 16 c l_{\alpha} a V_{2} V_{3}+32 c m_{\alpha} V_{2} V_{3}+32 c m_{\alpha} a b V_{2 w} \Omega_{1}+16 c l_{\alpha} b a^{2} V_{2 w} \Omega_{1}+ \\
& 32 c m_{\alpha} V_{2 w} V_{3}+16 c l_{\alpha} a V_{2} V_{3 w}+16 c l_{\alpha} a V_{2 w} V_{3 w}+8 c l_{\alpha} V_{2 w} V_{3}+ \\
& 8 c l_{\alpha} V_{2 w} V_{3 w}+16 c l_{\alpha} a V_{2 w}\left(\frac{1}{2} \sum_{n=1}^{N} b_{n} \lambda_{n}\right)+16 c l_{\alpha} a V_{2}\left(\frac{1}{2} \sum_{n=1}^{N} b_{n} \lambda_{n}\right)+ \\
& 16 c l_{\alpha} b a^{2} V_{2} \Omega_{1}+16 c l_{\alpha} a V_{2 w} V_{3}+8 c l_{\alpha} V_{2 w}\left(\frac{1}{2} \sum_{n=1}^{N} b_{n} \lambda_{n}\right)+8 c l_{\alpha} a b \dot{V}_{3}+ \\
& 8 c l_{\alpha} b^{2} a^{2} \dot{\Omega}_{1}+32 c m_{\alpha} V_{2 w} V_{3 w}-16 c m_{\alpha} b \Omega_{1} V_{2 w}+32 c m_{\alpha} a b V_{2} \Omega_{1}+ \\
& \left.16 c m_{\alpha} a b^{2} \dot{\Omega}_{1}-16 c m_{\alpha} b \Omega_{1} V_{2}+32 c m_{\alpha} V_{2 w}\left(\frac{1}{2} \sum_{n=1}^{N} b_{n} \lambda_{n}\right)\right)
\end{aligned}
$$


It should be noted that, for the sake of simplicity in Eqs. 2.26-2.28, the superscript $B$ on the variables is dropped. These equations give the aerodynamic loads on the blade in terms of the velocity variables and inflow states that are defined in the deformed structural frame. These aerodynamic loads along with gravity and structural damping loads are used in the next chapter to model the external loads on a helicopter rotor blade during engage/disengage operations. 


\section{Chapter 3}

\section{Dynamic Modelling}

In this chapter, the governing dynamic equations of a helicopter rotor blade during engage/disengage operations are developed. This chapter begins with the structural modelling of a blade as a nonlinear beam-like structure and continues on to model actuation loads in the blade. Then, the external loads including gravity, structural damping and aerodynamic loads on the blade are introduced and the aerodynamic inflow and wind effects are presented. In the end, equations from different sources are combined and the resultant set of control-centric equations is assembled for controller design in the next chapter.

\subsection{Structural Modelling}

In this research, a helicopter blade is assumed to be a nonlinear beam subject to large deformations and small strains. Under this assumption, the Intrinsic Beam Model developed by Hodges and colleagues $[24,28]$ is employed. This model was originally developed to analyze composite helicopter rotor blades, and can be used to analyze all beam-like structures. The model exploits one-dimensional characteristics of the beam in which all in-plane and out-of-plane deformations/warping effects are assumed to be captured in the beam cross sectional flexibility/stiffness matrices. This 
one-dimensional model was developed by applying Hamilton's principle to deformable structures. This formulation is called intrinsic, because it is independent of the choice of displacement and rotation variables. In other words, instead of displacement and rotation variables, linear and angular velocities appear in the formulation. It is also called geometrically exact, because the finite rotation of the cross-sectional frame in this model is treated exactly, removing the assumption of small angle rotations that form the basis of truncation beam models [28].

The main reason for employing the nonlinear intrinsic equations in this research is their analytical form and compactness. They are also easily reducible to the statespace form through an arbitrary discretization method. Moreover, they are applicable to anisotropic, non-uniform, twisted, and curved beams. These characteristics provide an ideal model of an active composite rotor blade suitable for control purposes.

\subsubsection{Intrinsic Structural Dynamic Equations}

Fig. 3.1 shows a schematic representation of an initially-twisted and curved blade undergoing deformation and warping. Frame $b$ identifies the cross section of the undeformed blade with initial twist/curvature whereas frame $B$ identifies the deformed cross section of this blade. Both frames of reference are orthogonal. Axis 1 is along the blade length, and axes 2 and 3 lie in the blade cross section. The geometrically-exact intrinsic equations for the dynamics of the blade, can be expressed as [28]:

$$
\begin{aligned}
F^{\prime}+(\widetilde{k+\kappa}) F+f & =\dot{P}+\tilde{\Omega} P \\
M^{\prime}+(\widetilde{k+\kappa}) M+\left(\widetilde{e_{1}+\gamma}\right) F+m & =\dot{H}+\tilde{\Omega} H+\tilde{V} P \\
V^{\prime}+(\widetilde{k+\kappa}) V+\left(\widetilde{e_{1}+\gamma}\right) \Omega & =\dot{\gamma} \\
\Omega^{\prime}+(\widetilde{k+\kappa}) \Omega & =\dot{\kappa}
\end{aligned}
$$




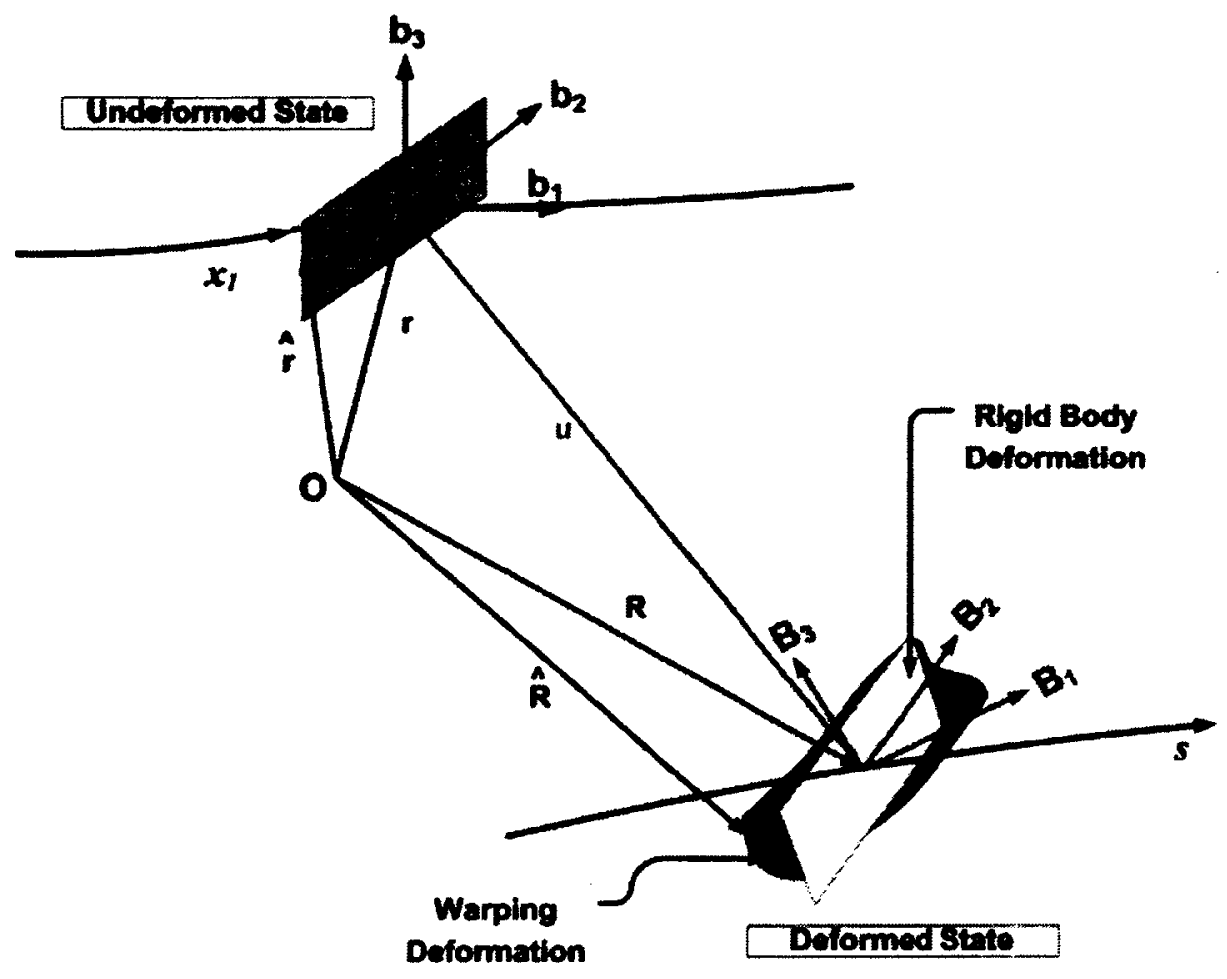

Figure 3.1: Schematic of a beam undergoing deformation and warping [3].

where:

- $F$ and $M$ are the vectors of internal forces and moments in the blade, defined in the $B$ frame;

- $V$ and $\Omega$ are the vectors of linear and angular velocity of the blade, defined in the $B$ frame;

- $P$ and $H$ are the vectors of linear and angular momentum of the blade, defined in the $B$ frame, and can be related to the internal forces and moments through cross-sectional constitutive relations;

- $\gamma$ and $\kappa$ are the vectors of strain and twist/curvature in the blade;

- $k$ is the vector of initial twist/curvature of the blade;

- $e_{1}=\{1 ; 0 ; 0\}$ is the unit vector along axis 1 ; 
- $f$ and $m$ are the external forces and moments per unit length due to gravity, aerodynamic, and structural damping loads defined in the $B$ frame;

- the prime operator (') takes the derivative of a variable with respect to the beam reference line (spatial derivative);

- the dot operator $\left({ }^{\circ}\right)$ takes the derivative of a variable with respect to time (temporal derivative); and

- the skew-symmetric tilde operator $\left(^{\sim}\right)$ transforms a vector $\{a\}$ to a matrix $\tilde{a}$ such that when multiplied by another vector $\{b\}$, the result would be $\tilde{a}\{b\}=$ $\{a\} \times\{b\}$.

It must be noted that the first two equations are the dynamic equations, whereas the last two ones are the intrinsic kinematic equations. To solve these four sets of partial differential equations (PDEs), four sets of boundary values are required. These boundary values are the internal loads at the blade tip, $F^{L}$ and $M^{L}$, and the linear and angular velocities of the blade at its root, $V^{0}$ and $\Omega^{0}$. Therefore, the following four sets of boundary value constraint equations have to be added to the intrinsic equations.

$$
\begin{aligned}
F(L) & =F^{L} \\
M(L) & =M^{L} \\
V(0) & =V^{0} \\
\Omega(0) & =\Omega^{0}
\end{aligned}
$$




\subsubsection{Constitutive Relations}

The primary variables $F, M, V$, and $\Omega$ and the secondary variables $\gamma, \kappa, P$, and $H$ of a rotor blade are related through cross-sectional constitutive relations [3], such that:

$$
\begin{gathered}
\left\{\begin{array}{c}
\gamma \\
\kappa
\end{array}\right\}=\left[\begin{array}{rr}
R & S \\
S^{T} & T
\end{array}\right]\left\{\begin{array}{c}
F+F^{a c t} \\
M+M^{a c t}
\end{array}\right\} \\
\left\{\begin{array}{l}
P \\
H
\end{array}\right\}=\left[\begin{array}{ll}
G & Q \\
Q^{T} & I
\end{array}\right]\left\{\begin{array}{c}
V \\
\Omega
\end{array}\right\}
\end{gathered}
$$

where $F^{a c t}$ and $M^{a c t}$ are the actuation forces and moments in the blade that will be discussed in the next section and are equal to zero for passive blades; $R, S$, and $T$ are matrices of cross-sectional flexibility coefficients; and $G, Q$, and $I$ are matrices of mass per unit length of the blade, first mass moments of inertia per unit length, and second mass moments of inertia per unit length as given by:

$$
G=\left[\begin{array}{ccc}
\bar{m} & 0 & 0 \\
0 & \bar{m} & 0 \\
0 & 0 & \bar{m}
\end{array}\right], Q=\left[\begin{array}{ccc}
0 & \bar{m} \xi_{3} & -\bar{m} \xi_{2} \\
-\bar{m} \xi_{3} & 0 & 0 \\
\bar{m} \xi_{2} & 0 & 0
\end{array}\right], I=\left[\begin{array}{ccc}
\bar{I}_{2}+\bar{I}_{3} & 0 & 0 \\
0 & \bar{I}_{2} & \bar{I}_{23} \\
0 & \bar{I}_{23} & \bar{I}_{3}
\end{array}\right]
$$

In the above matrices, $\bar{m}$ is the mass per unit length of the blade; $\xi_{1}, \xi_{2}$ and $\xi_{3}$ are the cross-sectional mass center offset of the blade, or the location of the mass center with respect to the blade reference line; $I_{i}$ is the mass moment of inertia about axis $i$; and $I_{i j}$ is the product of inertia about axes $i$ and $j$. It should be noted that, if the 
center of mass is located at the origin of the aerodynamic frame (mid-chord), then $\xi_{1}=\xi_{3}=0$ and $\xi_{2}=-a b$, where $a$ and $b$ are defined in the previous chapter. In the case of varying cross-sectional shapes and properties, the above flexibility and inertia matrices are functions of the blade reference line.

\subsection{Actuation Loads}

There are various methods proposed in the literature and industry to incorporate smart structures into helicopter rotor blades [29]. In this research, the Integral Active Twist (IAT) method developed by a previous member of the Advanced Dynamics Group (ADG) at Carleton University is utilized. As shown in Fig. 3.2, the general idea of IAT is to use some Active Fibre Composite (AFC) or Micro Fibre Composite (MFC) plies at different ply angles in the composite blade. Due to their structure, MFCs produce larger forces and displacements, are lighter, and have faster response time than AFCs.

Active fibres work based on the piezoelectric effect induced artificially in certain

ceramics such as Lead Zirconate Titanate (PZT). Direct piezoelectric effect refers to accumulation of electric charge in ceramics in response to applied mechanical stress (sensing effect). Reverse piezoelectricity refers to mechanical deformation or internal generation of a mechanical strain in the ceramic when subjected to an electrical field (actuation effect).

The static linear relation between the electric field applied to electrodes in active fibres and the actuation forces and moments produced in the blade cross sections is modelled in Ref. [3]. Therefore, only final results are presented here. Assuming linear electromechanical coupling in MFCs/AFCs, i.e.,

$$
\{\varepsilon\}=[d]\{E\}
$$




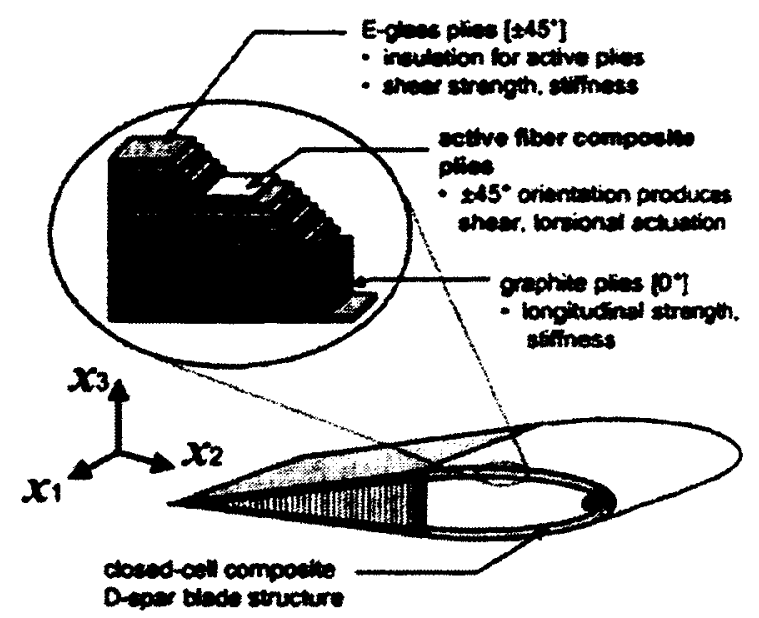

Figure 3.2: Typical laminate design of IAT rotor blade [ Courtesy of NASA Langley Research Center ].

it can be shown that:

$$
\begin{gathered}
F^{a c t}=Y^{F} V_{p} \\
M^{a c t}=Y^{M} V_{p}
\end{gathered}
$$

where, $\varepsilon$ is the induced strain vector, $[d]$ is the electromechanical coupling matrix, $E$ is the electric field across MFCs/AFCs in the blade, $V_{p}$ is the voltage along MFCs/AFCs in the blade, and $Y^{F}$ and $Y^{M}$ are piezoelectric field coefficient vectors.

Although the above linear static equations are used to model the actuation forces and moments in this research, there are three important points that should be considered for further developments.

- The relationship between electric field and strain is linear only for low electric fields. As can be seen in Fig. 3.3, as the electric field increases the polarization along the piezo fibres saturates and domains expand and switch. This causes significant nonlinear hystresis behaviour that could be detrimental to using piezo actuators for control purposes at high electric fields. There are some methods 


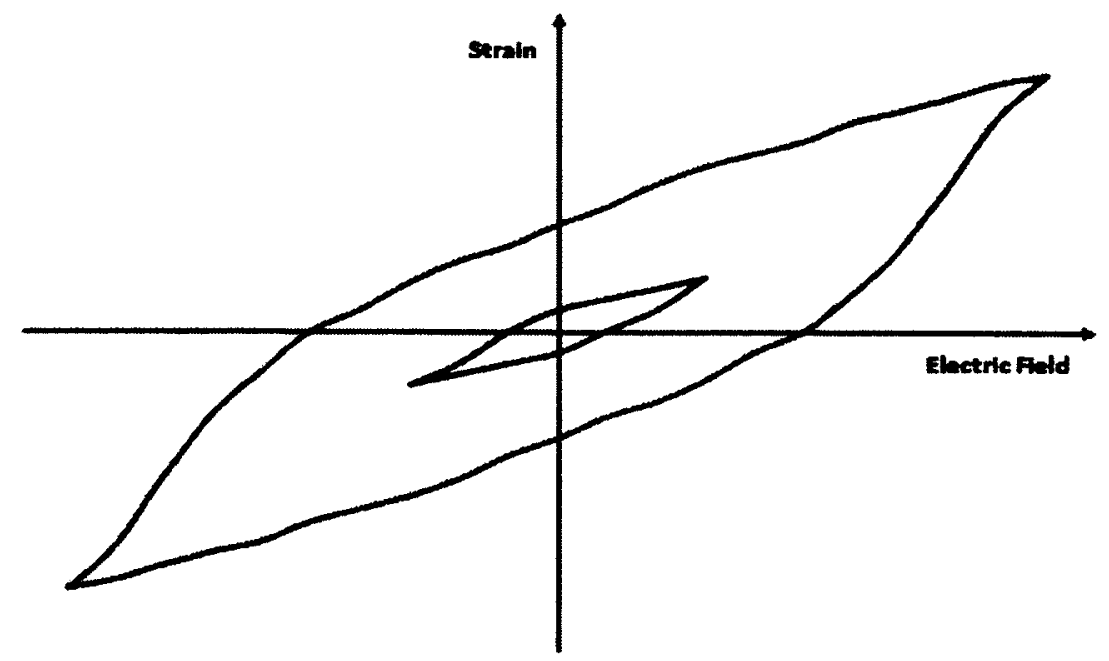

Figure 3.3: Hysteresis behaviour of a typical piezoelectric material.

to account for hysteresis [30]. However, these methods themselves add to the complexity of the closed-loop control system. Hence, they are not attractive to control system designers.

- Polarized fibres like all other materials have some electric capacitance and resistance when subjected to electric fields. Active elements such as capacitors change the static model of the piezo actuators drastically. Therefore for a more accurate control system design, the dynamics between the applied electric field and the induced actuation forces/moments produced by the actuators should be compensated.

- Piezo actuators provide relatively low control forces and need high input voltages to actuate. Such high voltages can be provided by voltage amplifiers on the static platforms. However on moving platforms such as a rotating helicopter blade, amplification is of practical concern.

Despite the above deficiencies, piezo actuators have some useful properties as follows [30]. 
- PZTs have fast response characteristics to the voltage and hence wide control bandwidth. This property makes them appropriate to control the structural vibrations where the dynamical modes are of high frequencies.

- They are light, compact, and can be relatively easily embedded in the structures. These characteristics are vital when it comes to the vibration control of active rotor blades where the available space and weight are highly limited.

As can be seen in the following sections, the time derivative of actuation forces and moments appear in the blade dynamic equations. To develop the control loop, the dynamics between these rates of applied actuation forces and moments $\left(\dot{F}^{\text {act }}, \dot{M}^{\text {act }}\right)$ and the commanded actuation signals $\left(u_{1}\right.$ and $\left.u_{2}\right)$ by the control system have to be modelled. However, as noted earlier, since the actuation model in this research does not include this dynamics, it is assumed that applied actuation forces and moments follow the commanded control signals with no delays or losses, i.e.,

$$
\begin{aligned}
& 0=\dot{F}^{a c t}-u_{1} \\
& 0=\dot{M}^{a c t}-u_{2}
\end{aligned}
$$

A physical interpretation of these equations is that the actuation power loss (difference between the input power to piezo actuators and what they transfer to the structure) is assumed to be negligible in this development.

\subsection{External Loads}

External forces and moments, $f$ and $m$, applied to a rotating blade come from different sources. They are caused by gravity loads $\left(f^{g r}, m^{g r}\right)$, structural damping loads $\left(f^{d}, m^{d}\right)$, and aerodynamic loads $\left(f^{a}, m^{a}\right)$. The aerodynamic loads can be due to 
steady windless aerodynamics $\left(f^{a s w l}, m^{a s w l}\right)$, wind aerodynamics $\left(f^{a w}, m^{a w}\right)$, and unsteady aerodynamics $\left(f^{a u}, m^{a u}\right)$. The resultant external forces and moments can thus be expressed as:

$$
\begin{aligned}
& f=f^{g r}+f^{d}+\underbrace{\left(f^{a s w l}+f^{a w}+f^{a u}\right)}_{f^{a}} \\
& m=m^{g r}+m^{d}+\underbrace{\left(m^{a s w l}+m^{a w}+m^{a u}\right)}_{m^{a}}
\end{aligned}
$$

It must be noted that due to strong coupling between various aerodynamic loads, these loads cannot be treated separately from a physical point of view. However, to design a controller, these loads need to be treated separately. The following sections provide more detail on derivation of these external loads.

\subsubsection{Gravity}

At high rotor RPMs, the blade weight is negligible compared to its aerodynamic lift. However, because BSP mostly happens at low rotor RPMs, blade weight is a significant source of load and has to be considered for correct modelling. Since the blade intrinsic equations are defined in the deformed frame, the gravitational loads have to be defined in this frame as well. To this end, a unit vector that identifies the direction of the gravitational field in the deformed frame is required.

In an inertial frame ( $I$ frame), the gravitational field is always directed opposite axis 3 with a constant acceleration of $9.81 \mathrm{~m} / \mathrm{s}^{2}$. Therefore the required unit vector in the inertial frame is denoted as ${ }^{I} g=\{0 ; 0 ;-1\}$. However while its magnitude always remains equal to one, the components of this unit vector in the deformed frame, which are required to express the direction of gravity in this frame, change with time. Ref. [31] gives the necessary equations to obtain this unit vector in the 
deformed frame as:

$$
\begin{aligned}
& 0=g^{\prime}+\widetilde{(k+\kappa) g} \\
& 0=\dot{g}+\widetilde{\Omega} g \\
& 0=|g|-1 \\
& 0=g(0)-g^{0}
\end{aligned}
$$

where $g$ is the unit vector defined in the deformed frame ( $B$ frame) and $g^{0}$ is the boundary value of the $g$ vector at the blade root. The abs operator $(||)$ in Eqn. 3.21 gives the magnitude of the vector. Using the above formulation, gravitational loads in the deformed frame can be expressed as:

$$
f^{g r}=G g_{m} g, \quad m^{g r}=Q g_{m} g
$$

As defined previously, $G$ and $Q$ are matrices of cross-sectional mass and cross-sectional first mass moment of inertia, respectively, and $g_{m}$ is the gravitational field acceleration $\left(g_{m}=9.81 \mathrm{~m} / \mathrm{s}^{2}\right)$.

\subsubsection{Structural Damping}

To include the structural damping loads in the intrinsic blade formulation, these loads are assumed to be proportional to the time rate of change of strains and curvatures, such that:

$$
\left\{\begin{array}{c}
f^{d} \\
m^{d}
\end{array}\right\}=-C\left\{\begin{array}{l}
\dot{\gamma} \\
\dot{\kappa}
\end{array}\right\}
$$

where, $C$ is the damping proportionality matrix. Assuming Rayleigh structural damping in the blade, the damping proportionality matrix can be written in terms of mass 
and stiffness matrices [3]:

$$
C=\bar{\eta}\left[\begin{array}{cc}
G & Q \\
Q^{T} & I
\end{array}\right]+\bar{\delta}\left[\begin{array}{cc}
R & S \\
S^{T} & T
\end{array}\right]^{-1}
$$

where $\bar{\eta}$ and $\bar{\delta}$ are the adjusting parameters that control the damping level of the low and high structural frequencies, respectively [3]. Substituting Eq. 3.25 in Eq. 3.24 and using Eq. 3.9 results in:

$$
\left\{\begin{array}{c}
f^{d} \\
m^{d}
\end{array}\right\}=\left[\begin{array}{cc}
J & N \\
B & W
\end{array}\right]\left\{\begin{array}{c}
\dot{F}+\dot{F}^{a c t} \\
\dot{M}+\dot{M}^{a c t}
\end{array}\right\}
$$

where

$$
\left[\begin{array}{ll}
J & N \\
B & W
\end{array}\right]=-C\left[\begin{array}{rr}
R & S \\
S^{T} & T
\end{array}\right]
$$

It should be noted that since the internal forces and moments and actuation loads are defined in the deformed frame, the obtained intrinsic damping loads are automatically defined in this frame.

\subsubsection{Aerodynamics}

In Chapter 2, the aerodynamic forces and moments on the blade are obtained in terms of velocity variables and inflow states that are defined in the deformed frame $B$. As long as the blade generates aerodynamic loads, the tangential component of the blade velocity is significantly larger than its vertical component. In other words, it can be assumed that $\left(V_{2}+V_{2 w}\right)$ is much larger than $\left(V_{3}+a b \Omega_{1}+\frac{1}{2} \sum_{n=1}^{N} b_{n} \lambda_{n}+V_{3 w}\right)$. Using 
this assumption, Eqs. $2.26-2.28$ can be simplified to:

$$
\begin{aligned}
f_{2}^{a}= & \rho b c l_{\alpha}\left(V_{3}+a b \Omega_{1}+\frac{1}{2} \sum_{n=1}^{N} b_{n} \lambda_{n}+V_{3 w}\right)^{2}-\rho b c d_{0}\left(V_{2}+V_{2 w}\right)^{2} \\
& -\rho b c l_{0}\left(V_{2}+V_{2 w}\right)\left(V_{3}+a b \Omega_{1}+\frac{1}{2} \sum_{n=1}^{N} b_{n} \lambda_{n}+V_{3 w}\right) \\
f_{3}^{a}= & -\rho b\left(c l_{\alpha}+c d_{0}\right)\left(V_{2}+V_{2 w}\right)\left(V_{3}+a b \Omega_{1}+\frac{1}{2} \sum_{n=1}^{N} b_{n} \lambda_{n}+V_{3 w}\right) \\
& +\frac{1}{2} \rho b^{2} c l_{\alpha}\left(V_{2}+V_{2 w}\right) \Omega_{1}-\frac{1}{2} \rho b^{2} c l_{\alpha}\left(\dot{V}_{3}+a b \dot{\Omega}_{1}\right)+\rho b c l_{0}\left(V_{2}+V_{2 w}\right)^{2} \\
m_{1}^{a}= & 2 \rho b^{2} c m_{0}\left(\left(V_{2}+V_{2 w}\right)^{2}+\left(V_{3}+a b \Omega_{1}+\frac{1}{2} \sum_{n=1}^{N} b_{n} \lambda_{n}+V_{3 w}\right)^{2}\right) \\
& -\rho b^{2} c m_{\alpha}\left(2 V_{2} V_{3}+2 V_{2} V_{3 w}+2 a b V_{2} \Omega_{1}+V_{2} \sum_{n=1}^{N} b_{n} \lambda_{n}+2 V_{2 w} V_{3}\right. \\
& +2 V_{2 w} V_{3 w}+2 a b V_{2 w} \Omega_{1}+V_{2 w} \sum_{n=1}^{N} b_{n} \lambda_{n}+b \dot{V}_{3}+a b^{2} \dot{\Omega}_{1}-b \Omega_{1} V_{2} \\
& \left.-b \Omega_{1} V_{2 w}\right)-\frac{1}{2} \rho b^{2} c l_{\alpha}\left((1+2 a) V_{2} V_{3}+(1+2 a) V_{2} V_{3 w}+(1+2 a) V_{2 w} V_{3}\right. \\
& +\frac{1}{2}(1+2 a) V_{2} \sum_{n=1}^{N} b_{n} \lambda_{n}+(1+2 a) V_{2 w} V_{3 w}+\frac{1}{2}(1+2 a) V_{2 w} \sum_{n=1}^{N} b_{n} \lambda_{n} \\
& \left.+b^{2}\left(a^{2}+\frac{1}{8}\right) \dot{\Omega}_{1}+2 b a^{2} V_{2} \Omega_{1}+2 b a^{2} V_{2 w} \Omega_{1}+a b \dot{V}_{3}\right)
\end{aligned}
$$

where all aerodynamic variables are defined in Chapter 2 . In the present work, three inflow states $\left(\lambda_{i}\right)$ are considered. Therefore $N=3$. Since all variables in intrinsic equations appear in vectors, the velocity variables in the above aerodynamic formulation are required to be cast as vectors, as well. This summarizes Eqs. 3.28-3.30 to 
the following equations.

$$
\begin{aligned}
f^{a s w l}= & e_{2}\left(V^{T} X_{f 2 V V} V+V_{w}^{T} X_{f 2 V_{w} V} V+V^{T} X_{f 2 V \Omega} \Omega+V_{w}^{T} X_{f 2 V \Omega} \Omega+\right. \\
& \left.\Omega^{T} X_{f 2 \Omega \Omega} \Omega\right)+e_{3}\left(V^{T} X_{f 3 V V} V+V_{w}^{T} X_{f 3 V_{w} V} V+V^{T} X_{f 3 V \Omega} \Omega+\right. \\
& \left.V_{w}^{T} X_{f 3 V \Omega} \Omega+X_{f 3 \dot{V}} \dot{V}+X_{f 3 \dot{\Omega}} \dot{\Omega}\right) \\
f^{a u}= & e_{2}\left(\lambda^{T} X_{f 2 \lambda \lambda} \lambda+V^{T} X_{f 2 V \lambda} \lambda+V_{w}^{T} X_{f 2 V \lambda} \lambda+\Omega^{T} X_{f 2 \Omega \lambda} \lambda\right)+ \\
& e_{3}\left(V^{T} X_{f 3 V \lambda} \lambda+V_{w}^{T} X_{f 3 V \lambda} \lambda\right) \\
f^{a w}= & e_{2}\left(V_{w}^{T} X_{f 2 V V} V_{w}\right)+e_{3}\left(V_{w}^{T} X_{f 3 V V} V_{w}\right) \\
m^{a s w l}= & e_{1}\left(V^{T} X_{m 1 V V} V+V_{w}^{T} X_{m 1 V_{w} V} V+V^{T} X_{m 1 V \Omega} \Omega+V_{w}^{T} X_{m 1 V \Omega} \Omega+\right. \\
& \left.\Omega^{T} X_{m 1 \Omega \Omega} \Omega+X_{m 1 \dot{V}} \dot{V} X_{m 1 \dot{\Omega}} \dot{\Omega}\right) \\
m^{a u}= & e_{1}\left(\lambda^{T} X_{m 1 \lambda \lambda} \lambda+\Omega^{T} X_{m 1 \Omega \lambda} \lambda+V^{T} X_{m 1 V \lambda} \lambda+V_{w}^{T} X_{m 1 V \lambda} \lambda\right) \\
m^{a w}= & e_{1}\left(V_{w}^{T} X_{m 1 V V} V_{w}\right)
\end{aligned}
$$

where $e_{1}=\{1 ; 0 ; 0\}, e_{2}=\{0 ; 1 ; 0\}$, and $e_{3}=\{0 ; 0 ; 1\}$ are unit vectors along axis 1,2 , and 3 , respectively; and $X_{i j . . . s}$ are aerodynamic coefficient matrices/vectors that can be found in Appendix B. At this point, it should be mentioned that the following aerodynamic matrices, $F_{\lambda}$ and $M_{\lambda}$, are used in Chapter 4 to design the controller:

$$
\begin{aligned}
F_{\lambda}= & e_{2}\left(\lambda^{T} X_{f 2 \lambda}+V^{T} X_{f 2 V \lambda}+V_{w}^{T} X_{f 2 V \lambda}+\Omega^{T} X_{f 2 \Omega \lambda}\right)+ \\
& e_{3}\left(V^{T} X_{f 3 V \lambda}+V_{w}^{T} X_{f 3 V \lambda}\right) \\
M_{\lambda}= & e_{1}\left(\lambda^{T} X_{m 1 \lambda \lambda}+\Omega^{T} X_{m 1 \Omega \lambda}+V^{T} X_{m 1 V \lambda}+V_{w}^{T} X_{m 1 V \lambda}\right)
\end{aligned}
$$




\subsubsection{Aerodynamic Inflow}

Unsteady aerodynamics has a significant effect on the aerodynamic loads and should be considered in modelling the loads on the blade. As mentioned earlier, for the purpose of this research three inflow states are chosen. Therefore, expanding Eq. 2.7, these inflow states can be expressed as:

$$
\begin{aligned}
0= & \frac{b}{2}\left(\left(3 b_{1}+2\right) \dot{\lambda}_{1}+\left(3 b_{2}-1\right) \dot{\lambda}_{2}+3 b_{3} \dot{\lambda}_{3}\right)+\left(V_{2}+V_{2 w}\right) \lambda_{1}+ \\
& b\left(2 \dot{V}_{3}+2 \dot{V}_{3 w}+2 b\left(a-\frac{1}{2}\right) \dot{\Omega}_{1}\right) \\
0= & \frac{b}{4}\left(\left(2 b_{1}+3\right) \dot{\lambda}_{1}+2 b_{2} \dot{\lambda}_{2}+\left(2 b_{3}-1\right) \dot{\lambda}_{3}\right)+\left(V_{2}+V_{2 w}\right) \lambda_{2}+ \\
& \frac{b}{2}\left(2 \dot{V}_{3}+2 \dot{V}_{3 w}+2 b\left(a-\frac{1}{2}\right) \dot{\Omega}_{1}\right) \\
0= & \frac{b}{6}\left(\left(2 b_{1}+2\right) \dot{\lambda}_{1}+\left(2 b_{2}+1\right) \dot{\lambda}_{2}+2 b_{3} \dot{\lambda}_{3}\right)+\left(V_{2}+V_{2 w}\right) \lambda_{3}+ \\
& \frac{b}{3}\left(2 \dot{V}_{3}+2 \dot{V}_{3 w}+2 b\left(a-\frac{1}{2}\right) \dot{\Omega}_{1}\right)
\end{aligned}
$$

where inflow coefficients $\left(b_{i}\right)$ are obtained from Eq. 2.6, and the velocity variables are defined in Chapter 2. The above set of equations can be summarized as:

$$
0=A_{i n f} \dot{\lambda}+B_{i n f} \lambda+C_{i n f}\left(\dot{V}+\dot{V}_{w}\right)+D_{i n f} \dot{\Omega}
$$

where

$$
A_{i n f}=\left[\begin{array}{ccc}
\frac{b}{2}\left(3 b_{1}+2\right) & \frac{b}{2}\left(3 b_{2}-1\right) & \frac{b}{2}\left(3 b_{3}\right) \\
\frac{b}{4}\left(2 b_{1}+3\right) & \frac{b}{4}\left(2 b_{2}\right) & \frac{b}{4}\left(2 b_{3}-1\right) \\
\frac{b}{6}\left(2 b_{1}+2\right) & \frac{b}{6}\left(2 b_{2}+1\right) & \frac{b}{6}\left(2 b_{3}\right)
\end{array}\right], C_{i n f}=\left[\begin{array}{ccc}
0 & 0 & 2 b \\
0 & 0 & b \\
0 & 0 & \frac{2}{3} b
\end{array}\right]
$$




$$
\begin{gathered}
B_{i n f}=\left[\begin{array}{ccc}
V_{2}+V_{2} w & 0 & 0 \\
0 & V_{2}+V_{2} w & 0 \\
0 & 0 & V_{2}+V_{2} w
\end{array}\right], D_{i n f}=\left[\begin{array}{ccc}
2 b^{2}\left(a-\frac{1}{2}\right) & 0 & 0 \\
b^{2}\left(a-\frac{1}{2}\right) & 0 & 0 \\
\frac{2}{3} b^{2}\left(a-\frac{1}{2}\right) & 0 & 0
\end{array}\right] \\
\lambda=\left\{\begin{array}{c}
\lambda_{1} \\
\lambda_{2} \\
\lambda_{3}
\end{array}\right\}, \quad V=\left\{\begin{array}{c}
V_{1} \\
V_{2} \\
V_{3}
\end{array}\right\}, \quad V_{w}=\left\{\begin{array}{c}
V_{1 w} \\
V_{2 w} \\
V_{3 w}
\end{array}\right\}, \quad \dot{\Omega}=\left\{\begin{array}{c}
\dot{\Omega}_{1} \\
\dot{\Omega}_{2} \\
\dot{\Omega}_{3}
\end{array}\right\}
\end{gathered}
$$

It must be noted that in Chapter 5 , for spatial discretization and subsequent numerical soultion of the equations, the following form of $B_{\text {inf }} \lambda$ is used.

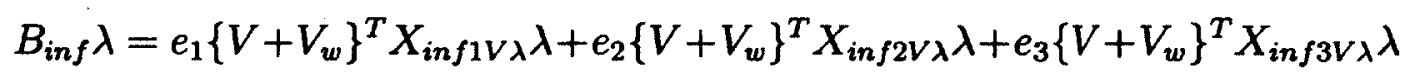

where

$$
X_{i n f 1 V \lambda}=\left[\begin{array}{ccc}
0 & 0 & 0 \\
1 & 0 & 0 \\
0 & 0 & 0
\end{array}\right], X_{i n f 2 V \lambda}=\left[\begin{array}{ccc}
0 & 0 & 0 \\
0 & 1 & 0 \\
0 & 0 & 0
\end{array}\right], X_{i n f 3 V \lambda}=\left[\begin{array}{ccc}
0 & 0 & 0 \\
0 & 0 & 1 \\
0 & 0 & 0
\end{array}\right]
$$

\subsubsection{Wind}

There are two methods to include the effect of wind in dynamic equations of interest. In the first method, assuming that the wind velocity is initially given in an inertial frame, similar to the gravity vector, this velocity vector has to be defined in the deformed frame. However, unlike the gravity vector that is always along axis 3 in 
the inertial frame, the wind velocity has nonzero components along all three inertial axes. Therefore, a direction cosine matrix is required to transform the wind velocity vector from an inertial frame to the deformed frame.

One way to obtain the terms of this direction cosine matrix is to treat each wind component individually. In this case, two sets of equations similar to Eqs. 3.19-3.21 are required: One to transform the unit vector ${ }^{I} e_{1}=\{1 ; 0 ; 0\}$ from an inertial frame to the deformed frame, and the other one to transform the unit vector ${ }^{I} e_{2}=\{0 ; 1 ; 0\}$ from an inertial frame to the deformed frame. The transformation of the unit vector ${ }^{I} e_{3}=\{0 ; 0 ; 1\}$ is addressed in Section 3.3.1. The components of the wind velocity in the deformed frame will be obtained by multiplying the magnitude of each wind component by the corresponding unit vector that is defined in the deformed frame, and adding them up. For example, the wind velocity vector ${ }^{I} V_{w}=\left\{V_{1 w} ; V_{2 w} ; V_{3 w}\right\}$ can be defined in the deformed frame as:

$$
{ }^{B} V_{w}=V_{1 w}{ }^{B} e_{1}+V_{2 w}^{B} e_{2}+V_{3 w}^{B} e_{3}
$$

where ${ }^{B} e_{i}$ is the transformed unit vector in deformed frame. This method although exact, increases the complexity of the dynamic model and as a result is not attractive from a control point of view.

The second method to include the effect of wind in the aerodynamic loads is to treat the wind velocity vector as a velocity boundary value. In this case, it is assumed that the magnitude and direction of the wind velocity is known at the blade root and the same wind velocity is distributed uniformly along the blade. This assumption decreases the complexity of the model significantly, yet gives the opportunity to consider the wind effect in the model. For these reasons, the second method is employed in this research. In the next section more information about the boundary conditions in the BSP problem will be given. 


\subsubsection{Boundary Conditions}

The boundary values of the intrinsic blade equations in the BSP are the linear velocity $V^{0}$ and the angular velocity $\Omega^{0}$ of the blade at its root. Since the blade is rotating in the non-inertial environment of the ship deck, to calculate the blade boundary values, the motion of the ship must be accounted for. In the current development, only hingeless rotors are considered. Therefore, using kinematic equations, the boundary values can be expressed in terms of the ship and the rotor hub motions as follows [3]:

$$
\begin{aligned}
\Omega^{0} & =C^{a h} \Omega_{z} \\
V^{0} & =C^{a h}\left[V_{z}+\left(\widetilde{\Omega_{z}} \vec{R}_{R}\right)\right] \\
\Omega_{z} & =C^{h s} C^{s i} \Omega_{s h i p}+\Omega_{h u b} \\
V_{z} & =C^{h s}\left[C^{s i} V_{\text {ship }}+C^{s i \Omega_{s h i p}}\left(\vec{R}_{H R}+\vec{R}_{H B}\right)\right]
\end{aligned}
$$

where:

- $C^{a h}, C^{h s}$, and $C^{s i}$, are the transformation matrices from the hub frame to the undeformed structural frame at the blade root, ship frame to hub frame, and inertial (ocean) frame to ship frame, respectively;

- $\vec{R}_{R}$ is the the blade root position vector in the hub frame and $\vec{R}_{H R}+\vec{R}_{H B}$ is the hub position vector in the inertial frame;

- $\Omega_{s h i p}$ is the ship angular velocity in the inertial frame and $\Omega_{h u b}$ is the hub angular velocity in the hub frame that can be summarized as:

$$
\Omega_{h u b}=\left\{\begin{array}{c}
0 \\
0 \\
\Omega_{h u b}^{e n g}
\end{array}\right\} \text { or }\left\{\begin{array}{c}
0 \\
0 \\
\Omega_{h u b}^{d i s}
\end{array}\right\}
$$


where $\Omega_{h u b}^{e n g}$ and $\Omega_{h u b}^{d i s}$ are the hub angular velocity during the engage and disengage operations, respectively.

In the literature, to study the BSP, the hub angular velocity during the engage/disengage operations is assumed to vary as a single offset trigonometric function. As an example, the engage/disengage profiles for an H-46 helicopter used in Ref. [6] are:

$$
\begin{gathered}
\Omega_{\text {hub }}^{\text {eng }}=\Omega_{\text {hub }}^{\max }\left\{\begin{array}{cc}
\frac{1}{2}\left\{\cos \left[\frac{3 \pi}{10}\left(t-t_{\text {eng }}\right)+\pi\right]+1\right\} & \forall t_{\text {eng }} \leq t \leq\left(t_{\text {eng }}+\frac{10}{3}\right) \\
1 & \forall t>\left(t_{\text {eng }}+\frac{10}{3}\right)
\end{array}\right\} \\
\Omega_{\text {hub }}^{\text {dis }}=\Omega_{\text {hub }}^{\max }\left\{\begin{array}{cc}
\frac{1}{2}\left\{\cos \left[\frac{3 \pi}{10}\left(t-t_{d i s}\right)\right]+1\right\} & \forall t_{d i s} \leq t \leq\left(t_{\text {dis }}+\frac{10}{3}\right) \\
0 & \forall t>\left(t_{\text {dis }}+\frac{10}{3}\right)
\end{array}\right\}
\end{gathered}
$$

where $\Omega_{h u b}^{\max }$ is the maximum nominal hub angular velocity during engage/disengage operation, $t_{e n g}$ is the engagement time, and $t_{d i s}$ is the disengagement time. These profiles are shown in Fig. 3.4 for engage/disengage times of $t_{e n g}=t_{d i s}=1 \mathrm{~s}$.

\subsection{Total Governing Equations}

As outlined in Ref. [25], there are two possible options for choosing the primary and secondary structural and dynamic variables $P, H, F, M, V, \Omega, \gamma$, and $\kappa$. For the purpose of this research, due to the fact that the cross sectional flexibility matrix of the blade may have some zero arrays (zero shear flexibility), or in other words the dynamical system may be a stiff one, $F, M, V$, and $\Omega$ are chosen as primary variables. Therefore, expressing the secondary variables $P, H, \gamma$, and $\kappa$ in terms of the primary variables in Eqs. 3.1-3.4, and 3.19 using constitutive relations, and including gravity 


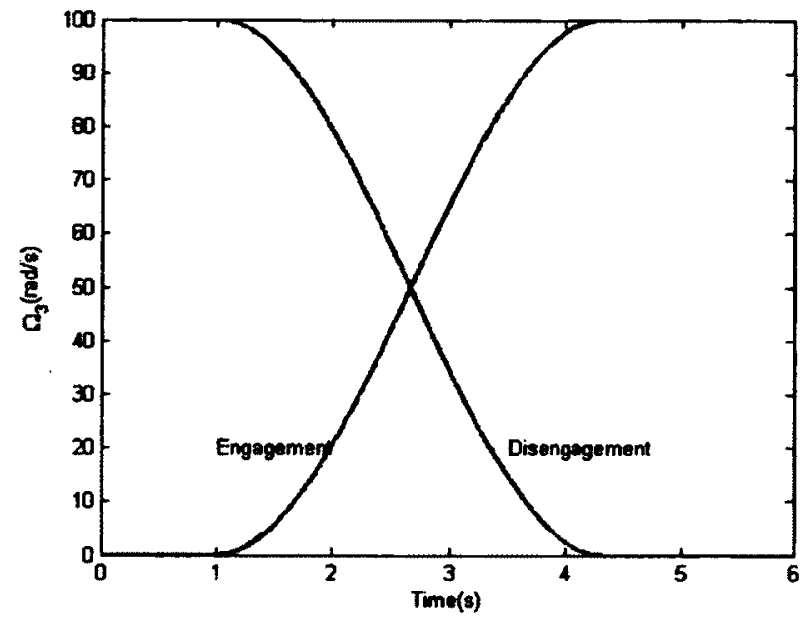

Figure 3.4: Typical engage/disengage profile.

(Eqs. 3.20 and 3.21), control (Eqs. 3.15 and 3.16), aerodynamic inflow (Eqs. 3.35), and boundary value constraints (Eqs. $3.5-3.8$ ), the complete formulation becomes: 


$$
\begin{aligned}
& 0=G \dot{V}+Q \dot{\Omega}-F^{\prime}-\tilde{k} F+\tilde{\Omega}(G V+Q \Omega)-\left[S^{T}\left(\widetilde{F+F^{a c t}}\right)+T\left(\widetilde{M+M^{a c t}}\right)\right] F \\
& -G g_{m} g-J\left(\dot{F}+\dot{F}^{a c t}\right)-N\left(\dot{M}+\dot{M}^{a c t}\right)-f^{a} \\
& 0=Q^{T} \dot{V}+I \dot{\Omega}-M^{\prime}-\tilde{k} M-\tilde{e_{1}} F+\tilde{\Omega}\left(Q^{T} V+I \Omega\right)+\tilde{V}(G V+Q \Omega) \\
& -\left[S^{T}\left(\widetilde{F+F^{a c t}}\right)+T\left(\widetilde{M+M^{a c t}}\right)\right] M-\left[R\left(\widetilde{F+F^{a c t}}\right)+S\left(\widetilde{M+M^{a c t}}\right)\right] F \\
& -Q g_{m} g-B\left(\dot{F}+\dot{F}^{a c t}\right)-W\left(\dot{M}+\dot{M}^{a c t}\right)-m^{a} \\
& 0=R\left(\dot{F}+\dot{F}^{a c t}\right)+S\left(\dot{M}+\dot{M}^{\text {act }}\right)-V^{\prime}-\tilde{k} V-\tilde{e_{1}} \Omega \\
& -\left[S^{T}\left(\widetilde{F+F^{a c t}}\right)+T\left(\widetilde{M+M^{a c t}}\right)\right] V-\left[R\left(\widetilde{F+F^{a c t}}\right)+S\left(\widetilde{M+M^{a c t}}\right)\right] \Omega \\
& 0=S^{T}\left(\dot{F}+\dot{F}^{a c t}\right)+T\left(\dot{M}+\dot{M}^{a c t}\right)-\Omega^{\prime}-\tilde{k} \Omega \\
& -\left[S^{T}\left(\widetilde{F+F^{a c t}}\right)+T\left(\widetilde{M+M^{a c t}}\right)\right] \Omega \\
& 0=F(L)-F^{L} \\
& 0=M(L)-M^{L} \\
& 0=V(0)-V^{0} \\
& 0=\Omega(0)-\Omega^{0} \\
& 0=g^{\prime}+\tilde{k} g+\left[S^{T}\left(\widetilde{F+F^{a c t}}\right)+T\left(\widetilde{M+M^{a c t}}\right)\right] g \\
& 0=\dot{g}+\widetilde{\Omega} g \\
& 0=|g|-1 \\
& 0=g(0)-g^{0} \\
& 0=A_{i n f} \dot{\lambda}+B_{i n f} \lambda+C_{i n f}\left(\dot{V}+\dot{V}_{w}\right)+D_{i n f} \dot{\Omega} \\
& 0=\dot{F}^{\text {act }}-u_{1} \\
& 0=\dot{M}^{\text {act }}-u_{2}
\end{aligned}
$$

The above formulation comprises 43 mixed partial differential equations (PDEs), ordinary differential equations (ODEs), and algebraic equations (AEs) with 24 state 
variables as components of $F, M, F^{\text {act }}, M^{\text {act }}, V, \Omega, g$, and $\lambda$, and 6 control inputs $u_{1}$ and $u_{2}$. In the next chapter, the control loop will be closed by designing the control inputs $u_{1}$ and $u_{2}$ as functions of the state variables, to decrease the blade tip vertical displacements or increase the blade tip clearance from the ship deck. In Chapter 5, the resultant closed-loop equations will be discretized in the space domain, by applying the Galerkin Spectral discretization method. 


\section{Chapter 4}

\section{Control System Design}

There are different methods to design nonlinear feedback controllers for small-scale nonlinear systems. All these methods rely on the analytical modelling, and are practically applicable only to systems having few state variables [32-34]. For large-scale nonlinear systems with many state variables such as structural systems, the best possible controllers, if not the only ones, are the controllers that are designed based on the Lyapunov stability theorems.

Lyapunov stability theorems are widely used to prove the stability of dynamical systems as well as designing stabilizing controllers for nonlinear systems. Based on these theorems, if one can find a special function called Lyapunovfunction for a dynamical system, the stability of that system is guaranteed. Practically speaking, in most cases, the energy of a system or a measure of the energy in a system, could be a candidate Lyapunov function.

In the previous chapter, the BSP was modelled as an analytical large-scale dynamical system with time-varying boundary conditions. This provides the main motivation to use the Lyapunov stability theorems to design the BSP controller. This chapter begins with reviewing Lyapunov stability theorems for nonlinear systems and continues on to propose the candidate BSP Lyapunov function. Then, a controller designed based on the law of conservation of energy proves that the proposed Lyapunov 
function is a stabilizing Lyapunov function for the BSP problem.

\subsection{Lyapunov Stability for Autonomous Dynami- cal Systems}

In this section, following a brief review of necessary definitions applicable to autonomous and non-autonomous dynamical systems, the Lyapunov stability theorems for these systems are outlined [32].

\subsubsection{Definitions}

- Lipshcitz condition: Consider the autonomous system

$$
\dot{x}=f(x)
$$

where $f: D \longrightarrow R^{n}$ is piecewise continuous in $t$ and $D$ is the domain. This function is said to satisfy the Lipschitz condition, if

$$
\|f(x)-f(y)\| \leq L\|x-y\|
$$

where $x, y \in D$ and $L$ is the Lipschitz constant. In the following definition, without loss of generality, the equilibrium point is assumed at the origin $\left(x^{e}=0\right)$ of the coordinate system.

- Equilibrium point: The origin $x=0 \in D$ is an equilibrium point of the autonomous system (4.1) if it has the property that whenever the state of the system starts at $x=0$, it will remain at $x=0$ for all future time.

- Stability: The equilibrium point $x=0$ of (4.1) is: 
- stable if, for any arbitrarily small $\varepsilon>0$, there is a $\delta=\delta(\varepsilon)>0$ such that $\|x(0)\|<\delta \Longrightarrow\|x(t)\|<\varepsilon, \forall t \geq 0$

- asymptotically stable if it is stable and $\delta$ can be chosen such that $\|x(0)\|<$ $\delta \Longrightarrow \lim _{t \rightarrow \infty} x(t)=0$.

- unstable if it is not stable.

- Positive Definite (P.D.): A Continuous scalar function $V(x): D \longrightarrow R$ of $x \in D$, with continuous partial derivatives with respect to all arguments, is said to be positive definite in $\mathrm{D}$ if

(i) $V(0)=0, \quad 0 \in D$

(ii) $V(x)>0 \quad \forall x \neq 0, x \in D$

- Negative Semi - Definite (N.S.D.): $V(x)$ is said to be negative semi-definite in $\mathrm{D}$ if

(i) $V(0)=0, \quad 0 \in D$

(ii) $V(x) \leq 0 \quad \forall x \neq 0, x \in D$

\subsubsection{Lyapunov Stability Theorem for Autonomous Systems}

For autonomous systems there is one main Lypunov stability theorem as follows:

- Stability for Autonomus Systems: Assume that the origin $x=0$ is an equilibrium point for the autonomous system (4.1) and $D \subset R^{n}$ be a domain containing $x=0$. If in a neighbourhood $D$ of $x=0$ there exists a continuously differentiable function $V(x): D \longrightarrow R$ such that

(i) $V(x)$ is positive definite, and 
(ii) $\dot{V}(x)$ is negative semi-definite in $D$,

then $x=0$ is stable. Moreover, if $\dot{V}(x)$ is negative definite, then $x=0$ is asymptotically stable.

\subsection{Lyapunov Stability for Non-autonomous Sys- tems}

\subsubsection{Definitions}

- Lipshcitz condition: Consider the non-autonomous system

$$
\dot{x}=f(x, t)
$$

where $f: D \times[0, \infty) \longrightarrow R^{n}$ is piecewise continuous in $t$. This function is said to satisfy the Lipschitz condition, if

$$
\|f(x, t)-f(y, t)\| \leq L\|x-y\|
$$

where $x, y \in D$ and $L$ is the Lipschitz constant. In the following definition, without loss of generality, the equilibrium point is assumed at the origin $\left(x^{e}=0\right)$ of the coordinate system.

- Equilibrium point: The origin $x=0 \in D$ is an equilibrium point of the nonautonomous system (4.2) at $t=t_{0}$ if

$$
f(0, t)=0 \forall t \geq t_{0}
$$

- Stability: The equilibrium point $x=0$ of $(4.2)$ is: 
- stable if, for any arbitrarily small $\varepsilon>0$, there is a $\delta=\delta\left(\varepsilon, t_{0}\right)>0$ such that

$$
\left\|x\left(t_{0}\right)\right\|<\delta \Longrightarrow\|x(t)\|<\varepsilon, \forall t \geq t_{0} \geq 0
$$

- uniformly stable if, for any arbitrarily small $\varepsilon>0$, there is a $\delta=\delta(\varepsilon)>0$, independent of $t_{0}$, such that (4.3) is satisfied.

- asymptotically stable if it is stable and there is a positive constant $c=c\left(t_{0}\right)$ such that $x(t) \rightarrow 0$ as $t \rightarrow \infty$, for all $\left\|x\left(t_{0}\right)\right\|<c$.

- uniformly asymptotically stable if it is uniformly stable and there is a positive constant $c$, independent of $t_{0}$ such that for all $\left\|x\left(t_{0}\right)\right\|<c, x(t) \rightarrow 0$ as $t \rightarrow \infty$, uniformly in $t_{0}$; that is for any $\eta>0$, there is a $T=T(\eta)>0$ such that

$$
\|x(t)\| \leq \eta, \forall t \geq t_{0}+T(\eta), \forall\left\|x\left(t_{0}\right)\right\|<c
$$

- globally uniformly asymptoticallystable if it is uniformly stable, and $\delta(\varepsilon)$ can be chosen to satisfy $\lim _{\varepsilon \rightarrow \infty} \delta(\varepsilon)=\infty$, and for each pair of positive numbers $\eta$ and $c$, there is $T=T(\eta)>0$ such that

$$
\|x(t)\| \leq \eta, \forall t \geq t_{0}+T(\eta, c), \forall\left\|x\left(t_{0}\right)\right\|<c
$$

- unstable if it is not stable.

- Positive Semi-Definite(P.S.D.): A continuous scalar function $W(x, t): D \times$ $R^{+} \longrightarrow R$ of two variables $x \in D, t \in R^{+}$, with continuous partial derivatives with respect to all arguments, is said to be positive semi-definite in $D$ if

(i) $W(0, t)=0 \quad \forall t \in R^{+}$

(ii) $W(x, t) \geq 0 \quad \forall x \neq 0, x \in D$

- Positive - Definite (P.D.): $W(x, t)$ is said to be positive-definite in $D$ if 
(i) $W(0, t)=0 \quad \forall t \in R^{+}$

(ii) There exists a time-invariant P.D. function $V_{1}(x)$ such that $V_{1}(x) \leq$ $W(x, t) \quad \forall x \in D$

- Decrescent: $W(x, t)$ is said to be decrescent if there exists a P.D. function $V_{2}(x)$ such that $W(x, t) \leq V_{2}(x)$

- Radially unbounded: $W(x, t)$ is said to be radially unbounded (R.U.), if $W(x, t) \longrightarrow \infty$ as $\|x\| \longrightarrow \infty$

\subsubsection{Lyapunov Stability Theorems for Non-autonomous Systems}

There are two main Lypunov stability theorems For non-autonomous systems as follows:

- Uniform Stability for Non-autonomus Systems: Assume that the origin $x=0$ is an equilibrium point for the non-autonomous system (4.2). If in a neighbourhood $D$ of $x=0$ there exists a continuously differentiable function $W(x, t): D \times[0, \infty) \longrightarrow R$ such that

(i) $W(x, t)$ is positive definite, and

(ii) $\dot{W}(x, t)$ is negative semi-definite in $\mathrm{D}$ along the trajectories of $(4.2)$,

then $x=0$ is stable. Furthermore, if $W(x, t)$ is decrescent, then $x=0$ is uniformly stable.

- Uniform Asymptotic Stability for Non-autonomus Systems: In the previous theorem, if

(i) $W(x, t)$ is positive definite, and 
(ii) $\dot{W}(x, t)$ is negative definite in $\mathrm{D}$ along trajectories of (4.2),

then $x=0$ is asymptotically stable. Furthermore, if $W(x, t)$ is decrescent, then $x=0$ is uniformly asymptotically stable.

\subsection{Candidate BSP Lyapunov Function}

The objective of the BSP control is to minimze the helicopter blade tip displacements in flapwise bending. This minimization can be treated as a trajectory control problem in which the deviation from the reference desired trajectory has to be minimized. The current actuation strategy (active twist) can only be effective in controlling the elastic displacements of the blade. Therefore, the reference desired trajectory in the current $\mathrm{BSP}$ control is the rigid-body motion of the blade during engage/disengage operations. However, if one needs to minimize the total rigid and elastic displacement of the blade, the reference desired trajectory is the case when the blade remains completely level while it is rotating with different angular velocities.

Minimization of any deviation can be achieved by minimizing the energy content in the blade that is associated with that deviation. This corresponds to interchanging the trajectory following problem in the original dynamics to a stability problem in the deviated (error) dynamics. Deviated energy can be a good Lyapunov candidate function. It is positive definite, so that by making its time derivative a negative definite function, we can get the required controller.

In this section, first the total energy of the blade is obtained in terms of the state variables, then it is shown that the law of conservation of energy will be fulfilled by multiplying the equations by some weightings. Next, the deviated energy is obtained by replacing the deviation of each state variable from its desired value in the law of conservation of energy equation. Finally, the BSP controller that stabilizes the dynamics of the deviation of the system with respect to its desired trajectory (zero 
blade flapwise displacements) is designed.

\subsubsection{Energy Balance Equation}

The law of conservation of energy states that the time rate of change of energy in a system is equal to the rate of work done by external loads on the system. In structural systems, energy is composed of kinetic and strain energy; and the loads could be structural damping, gravity, aerodynamic, and other applied loads.

The kinetic energy $(T)$ and the strain energy $\left(U^{s t}\right)$ of a rotating helicopter blade and their time rate of change can be expressed as [25]:

$$
\begin{aligned}
& T=\frac{1}{2} \int_{0}^{L}\left\{\begin{array}{c}
V \\
\Omega
\end{array}\right\}^{T}\left[\begin{array}{cc}
G & Q \\
Q^{T} & I
\end{array}\right]\left\{\begin{array}{c}
V \\
\Omega
\end{array}\right\} \mathrm{d} x \\
& \dot{T}=\int_{0}^{L}\left\{\begin{array}{c}
V \\
\Omega
\end{array}\right\}^{T}\left[\begin{array}{cc}
G & Q \\
Q^{T} & I
\end{array}\right]\left\{\begin{array}{c}
\dot{V} \\
\dot{\Omega}
\end{array}\right\} \mathrm{d} x=\int_{0}^{L}\left\{V^{T} \dot{P}+\Omega^{T} \dot{H}\right\} \mathrm{d} x \\
& U^{s t}=\frac{1}{2} \int_{0}^{L}\left\{\begin{array}{l}
\gamma \\
\kappa
\end{array}\right\}^{T}\left[\begin{array}{rr}
R & S \\
S^{T} & T
\end{array}\right]^{-1}\left\{\begin{array}{l}
\gamma \\
\kappa
\end{array}\right\} \mathrm{d} x \\
& \dot{U}^{s t}=\int_{0}^{L}\left\{\begin{array}{l}
\gamma \\
\kappa
\end{array}\right\}^{T}\left[\begin{array}{cc}
R & S \\
S^{T} & T
\end{array}\right]^{-1}\left\{\begin{array}{c}
\dot{\gamma} \\
\dot{\kappa}
\end{array}\right\} \mathrm{d} x \\
& =\int_{0}^{L}\left\{\left(F+F^{a c t}\right)^{T} \dot{\gamma}+\left(M+M^{a c t}\right)^{T} \dot{\kappa}\right\} \mathrm{d} x
\end{aligned}
$$

The rate of the work done by non-conservative structural damping loads $\left(P^{d a m}\right)$, non-conservative aerodynamic loads $\left(P^{a e r}\right)$ (including wind and unsteady inflow effects), and the conservative gravitational loads or the gravitational potential energy 
$\left(P^{g r}\right)$ of the blade can be expressed as:

$$
\begin{array}{r}
P^{a e r}=\int_{0}^{L}\left\{V^{T} f^{a w s l}+\Omega^{T} m^{a w s l}+V^{T} f^{a w}+\Omega^{T} m^{a w}+V^{T} F_{\lambda} \lambda+\Omega^{T} M_{\lambda} \lambda+\right. \\
\left.e^{T}\left(F_{\lambda}+\frac{M_{\lambda}}{a b}\right)\left[A_{i n f} \dot{\lambda}+B_{i n f} \lambda+C_{i n f}\left(\dot{V}+\dot{V}_{w}\right)+D_{i n f} \dot{\Omega}\right]\right\} \mathrm{d} x \\
P^{d a m}=\int_{0}^{L}\left\{V^{T}\left[-C_{11} \dot{\gamma}-C_{12} \dot{\kappa}\right]+\Omega^{T}\left[-C_{21} \dot{\gamma}-C_{22} \dot{\kappa}\right]\right\} \mathrm{d} x \\
P^{g r}=\int_{0}^{L}\left\{V ^ { T } G g _ { m } x \left(\widetilde{k+\kappa) g}+\Omega^{T} Q g_{m} x(\widetilde{k+\kappa)})+g^{T} G g_{m} x(\dot{g}+\tilde{\Omega} g)+\right.\right. \\
\left.g^{T} Q g_{m}(\dot{g}+\tilde{\Omega} g)+\left|V^{T} G g_{m}\right|[|g|-1]+\left|\Omega^{T} Q g_{m}\right|[|g|-1]\right\} \mathrm{d} x
\end{array}
$$

It should be noted that since gravity load is a conservative load, the rate of the work done by gravitational loads can be expressed as the time rate of corresponding potential energy. This means that, $P^{g r}$ in the above equations can be replaced by $-\dot{U}^{g r}$. However, since for controller design based on the Lyapunov stability theorems, a positive measure of energy in the system is required, the gravity effect is shown as a power induced in the system through the gravity forces/moments.

The power induced in the blade by actuation forces and moments ( $\left.P^{a c t}\right)$ and the amount of loss in this power $\left(P^{a w}\right)$ can be obtained from:

$$
\begin{aligned}
& P^{a c t}= \int_{0}^{L}\left\{F^{a c t}{ }^{T} \dot{\gamma}+M^{a c t} \dot{\kappa}^{T}\right\} \mathrm{d} x \\
&= \int_{0}^{L}\left\{F^{a c t^{T}}\left[V^{\prime}+(\widetilde{k+\kappa}) V+\left(\widetilde{e_{1}+\gamma}\right) \Omega\right]+M^{a c t^{T}}\left[\Omega^{\prime}+(\widetilde{k+\kappa}) \Omega\right]\right\} \mathrm{d} x \\
& P^{a w}=\int_{0}^{L}\left\{\left(R F^{a c t}+S M^{a c t}\right)^{T}\left[\dot{F}^{a c t}-u_{1}\right]+\right. \\
&\left.\left(S^{T} F^{a c t}+T M^{a c t}\right)^{T}\left[\dot{M}^{\text {act }}-u_{2}\right]\right\} \mathrm{d} x
\end{aligned}
$$

The power induced in the blade through the loads acting at the root and tip 
boundaries is:

$$
\begin{aligned}
P^{\text {bou }}= & V(L)^{T} F^{L}+\Omega(L)^{T} M^{L}+V(L)^{T} G L g_{m} g(L)+\Omega(L)^{T} Q L g_{m} g(L) \\
& -F(0)^{T} V^{0}-M(0)^{T} \Omega^{0}-V(0)^{T} G L g_{m} g^{0}-\Omega(0)^{T} Q L g_{m} g^{0} \\
& +V(0)^{T} G L g_{m} g(0)+\Omega(0)^{T} Q L g_{m} g(0)
\end{aligned}
$$

To satisfy the conservation of energy in an active blade one needs to obtain the following energy rate balance:

$$
\dot{T}+\dot{U}^{s t}=P^{a e r}+P^{g r}+P^{b o u}+P^{a c t}+P^{a w}+P^{d a m}
$$

where the left hand side is the time rate of change of the energy in the blade and the right hand side is the rate of work done by external loads and the boundary values. For passive blades, the actuation power $P^{a c t}$ and actuation power loss $P^{a w}$ are zero. Therefore, the energy conservation law is exactly satisfied if the structural damping is neglected, i.e.,

$$
\dot{T}+\dot{U}^{s t}=P^{a e r}+P^{g r}+P^{b o u}
$$

In the next section, the weighting functions required to fulfil the law of conservation of energy are introduced and the associated derivation is presented.

\subsubsection{Energy Consistent Weighting}

To obtain the the law of conservation of energy in the blade (Eq. 4.14), each equation in equation set $3.49 \mathrm{a}-3.49 \mathrm{n}$ need to be multiplied by an appropriate weighting function. Weighting functions make the resultant equations compatible, since all terms are either a kind of energy time derivative or a kind of power. Therefore, these equations can be added together to obtain the law of conservation of energy in the blade. To this end, it can be shown that the following weighting functions are to be used for 
each equation:

- $V^{T}$ for the first structural dynamics equation (Eq. 3.49a)

- $\Omega^{T}$ for the second structural dynamics equation (Eq. 3.49b)

- $\left(F+F^{A}\right)^{T}$ for the first kinematic equation (Eq. 3.49c)

- $\left(M+M^{A}\right)^{T}$ for the second kinematic equation (Eq. 3.49d)

- $V(L)^{T}$ for the force boundary value constraint at the tip (Eq. 3.49e)

- $\Omega(L)^{T}$ for the moment boundary value constraint at the tip (Eq. 3.49f)

- $\left(F(0)+F^{a c t}(0)\right)$ for the linear velocity boundary value constraint at the root (Eq. $3.49 \mathrm{~g})$

- $\left(M(0)+M^{a c t}(0)\right)$ for the angular velocity boundary value constraint at the root (Eq. $3.49 \mathrm{~h})$

- $\left(V^{T} G g_{m} x+\Omega^{T} Q g_{m} x\right)$ for the gravity unit vector spatial equation (Eq. 3.49i)

- $\left(g^{T} G g_{m} x+g^{T} Q g_{m}\right)$ for the gravity unit vector temporal equation (Eq. 3.49j)

- $\left(\left|V^{T} G g_{m}\right|+\left|\Omega^{T} Q g_{m}\right|\right)$ for the gravity unit vector magnitude constraint (Eq. $3.49 \mathrm{k})$

- $\left(V(0)^{T} G L g_{m}+\Omega(0)^{T} Q L g_{m}\right)$ for the gravity unit vector boundary constraint at the root (Eq. 3.491)

- $\left(e^{T} F_{\lambda}+e^{T} \frac{M_{\lambda}}{a b}\right)$ for the aerodynamic inflow equation (Eq. 3.49m)

- $\left(R F^{a c t}+S M^{a c t}\right)^{T}$ for the first control equation (force control) (Eq. 3.49n)

- $\left(S^{T} F^{a c t}+T M^{a c t}\right)^{T}$ for the second control equation (moment control) (Eq. 3.49o) 
All variables in these weighting functions are defined in previous sections. It should be noted that $L$ and 0 as the arguments of variables show the value that variables take at the blade tip and root, respectively.

In addition to fulfilling the law of conservation of energy in the blade, there are two other reasons to choose the above weighting functions. First, it will be seen in the next chapter that the same weighting functions can be used for discretization of the blade using the Galerkin Spectral discretization method. This capability simplifies the formulation significantly as the same weighting functions are used for both control design and discretization. Second, the main intrinsic structural equations (Eq. 3.49aEq. $3.49 \mathrm{~d}$ ) are derived using the concept of virtual work that is completely consistent with the selected weighting functions for them [28].

Applying these weighting functions to the corresponding equations and carrying out the spatial integration along the blade, and adding the resultant equations together, following result is obtained:

$$
\begin{aligned}
0=\int_{0}^{L}\{ & V^{T}\left[\dot{P}+\tilde{\Omega} P-F^{\prime}-(\widetilde{k+\kappa}) F-f\right] \\
& +\Omega^{T}\left[\dot{H}+\tilde{\Omega} H+\tilde{V} P-M^{\prime}-\widetilde{\left.(k+\kappa) M-\left(\widetilde{e_{1}+\gamma}\right) F-m\right]}\right. \\
& +\left(F+F^{a c t}\right)^{T}\left[\dot{\gamma}-V^{\prime}-(\widetilde{k+\kappa}) V-\left(\widetilde{e_{1}+\gamma}\right) \Omega\right] \\
& +\left(M+M^{a c t}\right)^{T}\left[\dot{\kappa}-\Omega^{\prime}-(\widetilde{k+\kappa}) \Omega\right] \\
& +\left(V^{T} G g_{m} x+\Omega^{T} Q g_{m} x\right)\left[-g^{\prime}-(\widetilde{k+\kappa}) g\right] \\
& +\left(g^{T} G g_{m} x+g^{T} Q g_{m}\right)[-\dot{g}-\widetilde{\Omega} g] \\
& +\left(\left|V^{T} G g_{m}\right|+\left|\Omega^{T} Q g_{m}\right|\right)[-|g|+1] \\
& +\left(e^{T} F_{\lambda}+e^{T} \frac{M_{\lambda}}{a b}\right)\left[-A_{i n f} \dot{\lambda}-B_{i n f} \lambda-C_{i n f}\left(\dot{V}+\dot{V}_{w}\right)-D_{i n f} \dot{\Omega}\right]
\end{aligned}
$$




$$
\begin{aligned}
& \left.\quad+\left(R F^{a c t}+S M^{a c t}\right)^{T}\left[\dot{F}^{a c t}-u_{1}\right]+\left(S^{T} F^{a c t}+T M^{a c t}\right)^{T}\left[\dot{M}^{a c t}-u_{2}\right]\right\} \mathrm{d} x \\
& -\left(F(0)+F^{a c t}(0)\right)^{T}\left[V(0)-V^{0}\right]-\left(M(0)+M^{a c t}(0)\right)^{T}\left[\Omega(0)-\Omega^{0}\right] \\
& +V(L)^{T}\left[F(L)-F^{L}\right]+\Omega(L)^{T}\left[M(L)-M^{L}\right] \\
& -\left(V(0)^{T} G L g_{m}+\Omega(0)^{T} Q L g_{m}\right)\left[g(0)-g^{0}\right]
\end{aligned}
$$

Using integration by parts, the integration of the terms involving $F^{\prime}, M^{\prime}$, and $g^{\prime}$ can be expanded as:

$$
\begin{gathered}
\int_{0}^{L}-\left(V^{T} F^{\prime}+\Omega^{T} M^{\prime}\right) \mathrm{d} x=-\left.V^{T} F\right|_{0} ^{L}-\left.\Omega^{T} M\right|_{0} ^{L}+\int_{0}^{L}\left(V^{T^{\prime}} F+\Omega^{T^{\prime}} M\right) \mathrm{d} x \\
\int_{0}^{L}-\left(V^{T} G g_{m} x+\Omega^{T} Q g_{m} x\right) g^{\prime} \mathrm{d} x \approx \\
-\left.V^{T} G g_{m} x g\right|_{0} ^{L}-\left.\Omega^{T} Q g_{m} x g\right|_{0} ^{L} \\
+\int_{0}^{L}\left(V^{T} g_{m} G+\Omega^{T} g_{m} Q\right) g \mathrm{~d} x
\end{gathered}
$$

The reason for the approximate equivalence in Eq. 4.18 is that $V$ and $\Omega$ are assumed to be approximately constant in the spatial integration. In general, this is not a valid assumption, because they change along the blade. However, since the resultant energy balance equations are used just to design the controller and the performance of the designed controller will be tested on the real system (original equations), this assumption is made to simplify the formulation. 
By substituting Eqs. 4.17 and 4.18 in Eq. 4.16, the latter becomes:

$$
\begin{aligned}
& 0=\int_{0}^{L}\{V^{T} \dot{P} \underbrace{+V^{T} \tilde{\Omega} P}_{1} \underbrace{+V^{T^{\prime}} F}_{2} \underbrace{-V^{T}(\widetilde{k+\kappa) F}}_{3}-V^{T} f^{a w s l}-V^{T} f^{a w}-V^{T} F_{\lambda} \lambda \\
& -V^{T}\left[-C_{11} \dot{\gamma}-C_{12} \dot{\kappa}\right] \underbrace{-V^{T} G g_{m} g}_{4}+\Omega^{T} \dot{H} \underbrace{+\Omega^{T} \tilde{\Omega} H}_{=0} \underbrace{+\Omega^{T} \tilde{V} P}_{1} \underbrace{+\Omega^{T^{\prime}} M}_{5} \\
& \underbrace{-\Omega^{T}(\widetilde{k+\kappa}) M}_{6} \underbrace{-\Omega^{T}\left(\widetilde{e_{1}+\gamma}\right) F}_{7}-\Omega^{T} m^{a w s l}-\Omega^{T} m^{a w}-\Omega^{T} M_{\lambda} \lambda \\
& -\Omega^{T}\left[-C_{21} \dot{\gamma}-C_{22} \dot{\kappa}\right] \underbrace{-\Omega^{T} Q g_{m} g}_{4}+\left(F+F^{a c t}\right)^{T} \dot{\gamma} \underbrace{-F^{T} V^{\prime}}_{2} \\
& \underbrace{-F^{T}(\widetilde{k+\kappa)})}_{3} \underbrace{-F^{T}\left(\widetilde{e_{1}+\gamma}\right) \Omega}_{7}+\left(M+M^{a c t}\right)^{T} \dot{\kappa} \underbrace{-M^{T} \Omega^{\prime}}_{5} \\
& +F^{a c t^{T}}\left[-V^{\prime}-(\widetilde{k+\kappa}) V-\left(\widetilde{e_{1}+\gamma}\right) \Omega\right]+M^{a c t}{ }^{T}\left[-\Omega^{\prime}-(\widetilde{k+\kappa}) \Omega\right] \\
& \underbrace{+V^{T} G g_{m} g}_{4} \underbrace{+\Omega^{T} Q g_{m} g}_{4} \underbrace{-M^{T}(\widetilde{k+\kappa}) \Omega}_{6}-V^{T} G g_{m} x(\widetilde{k+\kappa}) g \\
& -\Omega^{T} Q g_{m} x(\widetilde{k+\kappa}) g+\left|V^{T} G g_{m}\right|+\left|\Omega^{T} Q g_{m}\right|-\left|V^{T} G g_{m}\right||g| \\
& -\left|\Omega^{T} Q g_{m}\right||g|-\left(e^{T} F_{\lambda}+e^{T} \frac{M_{\lambda}}{a b}\right) A_{i n f} \dot{\lambda}-\left(e^{T} F_{\lambda}+e^{T} \frac{M_{\lambda}}{a b}\right) B_{i n f} \lambda \\
& -\left(e^{T} F_{\lambda}+e^{T} \frac{M_{\lambda}}{a b}\right) C_{i n f}\left(\dot{V}+\dot{V}_{w}\right)-\left(e^{T} F_{\lambda}+e^{T} \frac{M_{\lambda}}{a b}\right) D_{i n f} \dot{\Omega} \\
& \left.+\left(R F^{a c t}+S M^{a c t}\right)^{T}\left[\dot{F}^{a c t}-u_{1}\right]+\left(S^{T} F^{a c t}+T M^{a c t}\right)^{T}\left[\dot{M}^{\text {act }}-u_{2}\right]\right\} \mathrm{d} x \\
& +F(0)^{T} V^{0} \underbrace{-F(0)^{T} V(0)}_{8}-F^{a c t}(0)^{T}\left[V(0)-V^{0}\right] \\
& +M(0)^{T} \Omega^{0} \underbrace{-M(0)^{T} \Omega(0)}_{9}-M^{a c t}(0)^{T}\left[\Omega(0)-\Omega^{0}\right] \\
& -V(L)^{T} F^{L} \underbrace{+V(L)^{T} F(L)}_{10} \underbrace{+\Omega(L)^{T} M(L)}_{11}-\Omega(L)^{T} M^{L} \\
& -\left.V^{T} G g_{m} x g\right|_{0} ^{L}-\left.\Omega^{T} Q g_{m} x g\right|_{0} ^{L} \underbrace{-\left.V^{T} F\right|_{0} ^{L}}_{8,10} \underbrace{-\left.M^{T} \Omega\right|_{0} ^{L}}_{9,11} \\
& -\left(V(0)^{T} G L g_{m}+\Omega(0)^{T} Q L g_{m}\right)\left[g(0)-g^{0}\right]
\end{aligned}
$$


In Eq. 4.19, sets of variables with identical underbrace number cancel each other. Therefore, the resultant equation is:

$$
\begin{aligned}
& 0=\int_{0}^{L}\{\underbrace{V^{T} \dot{P}+\Omega^{T} \dot{H}}_{=\dot{T}} \underbrace{+\left(F+F^{a c t}\right)^{T} \dot{\gamma}+\left(M+M^{a c t}\right)^{T} \dot{\kappa}}_{=\dot{U} s t} \\
& \underbrace{-V^{T} f^{a w s l}-\Omega^{T} m^{a w s l}-V^{T} f^{a w}-\Omega^{T} m^{a w}-V^{T} F_{\lambda} \lambda-\Omega^{T} M_{\lambda} \lambda}_{=-P^{a e r}} \\
& \underbrace{+F^{a c t}{ }^{T}\left[-V^{\prime}-(\widetilde{k+\kappa}) V-\left(\widetilde{e_{1}+\gamma}\right) \Omega\right]+M^{\text {act }}{ }^{T}\left[-\Omega^{\prime}-(\widetilde{k+\kappa}) \Omega\right]}_{=-P^{\text {act }}} \\
& \underbrace{-V^{T} G g_{m} x(\widetilde{k+\kappa}) g-\Omega^{T} Q g_{m} x(\widetilde{k+\kappa}) g-\left(\left|V^{T} G g_{m}\right|+\left|\Omega^{T} Q g_{m}\right|\right)|g|}_{=\dot{U} g^{r} \text { or }-P^{g r}} \\
& \underbrace{+\left|V^{T} G g_{m}\right|+\left|\Omega^{T} Q g_{m}\right|}_{=\dot{U}^{g r} \text { or }-P^{g r}} \underbrace{-V^{T}\left[-C_{11} \dot{\gamma}-C_{12} \dot{\kappa}\right]-\Omega^{T}\left[-C_{21} \dot{\gamma}-C_{22} \dot{\kappa}\right]}_{=-P^{\text {dam }}} \\
& \underbrace{+\left(e^{T} F_{\lambda}+e^{T} \frac{M_{\lambda}}{a b}\right) A_{\text {inf }} \dot{\lambda}+\left(e^{T} F_{\lambda}+e^{T} \frac{M_{\lambda}}{a b}\right) B_{\text {inf }} \lambda}_{=-P^{\text {aer }}} \\
& \underbrace{+\left(e^{T} F_{\lambda}+e^{T} \frac{M_{\lambda}}{a b}\right) C_{i n f}\left(\dot{V}+\dot{V}_{w}\right)+\left(e^{T} F_{\lambda}+e^{T} \frac{M_{\lambda}}{a b}\right) D_{i n f} \dot{\Omega}}_{=-P^{\text {eer }}} \\
& \underbrace{+\left(R F^{a c t}+S M^{a c t}\right)^{T}\left[\dot{F}^{a c t}-u_{1}\right]+\left(S^{T} F^{a c t}+T M^{a c t}\right)^{T}\left[\dot{M}^{\text {act }}-u_{2}\right]}_{=P^{a w}}\} \mathrm{d} x \\
& \underbrace{-F^{\text {act }}(0)^{T}\left[V(0)-V^{0}\right]}_{\approx 0} \underbrace{-M^{\text {act }}(0)^{T}\left[\Omega(0)-\Omega^{0}\right]}_{\approx 0} \underbrace{+F(0)^{T} V^{0}+M(0)^{T} \Omega^{0}}_{=-P^{\text {bou }}} \\
& \underbrace{-V(L)^{T} F^{L}-\Omega(L)^{T} M^{L}-V(L)^{T} G L g_{m} g(L)-\Omega(L)^{T} Q L g_{m} g(L)}_{=- \text {Pbou }^{\text {bou }}} \\
& \underbrace{+V(0)^{T} G L g_{m} g^{0}+\Omega(0)^{T} Q L g_{m} g^{0}-V(0)^{T} G L g_{m} g(0)-\Omega(0)^{T} Q L g_{m} g(0)}_{=-P^{\text {bou }}}
\end{aligned}
$$

The above equation satisfies the law of energy conservation in an active blade, i.e. Eq. 4.14. Therefore, the chosen weighting functions give a reasonable resultant equation. 


\subsection{The BSP Lyapunov Controller}

In this section, the BSP Lyapunov controller is designed based on the energy balance equation derived in the previous section. By defining the state variable vector $X$ as a vector containing all state variables $F, M, V, \Omega, \Lambda$, and $g$, the deviation of this state vector from its desired value can be presented as:

$$
X^{*}=X-X^{d}
$$

where $X^{d}$ and $X$ are the desired and the actual state vectors, respectively. By replacing all state variables in terms of the desired and deviation values in the energy balance equation (Eq. 4.14), one can obtain:

$$
T+U^{s t}=T^{d}+U^{s t^{d}}+T^{*}+U^{s t^{*}}
$$

where $(\cdot)^{d}$ is the desired value and $(\cdot)^{*}$ is the difference between the actual and the desired value.

As outlined earlier, the desired trajectory corresponds to the case where the displacement of the structural deformed frame with respect to the structural undeformed frame is zero. This displacement and the associated rotation matrices can be obtained from the following equations [28]:

$$
\begin{aligned}
& C^{b a^{\prime}}=-\widetilde{k} C^{b a} \\
& C^{B a^{\prime}}=-(\widetilde{k+\kappa}) C^{B a} \\
& C^{B b}=C^{B a} \times C^{b a^{T}} \\
& \gamma=C^{B b}\left(e_{1}+U^{\prime}+\widetilde{k} U\right)-e_{1}
\end{aligned}
$$


where $C^{b a}$ is the rotation matrix from the untwisted, uncurved, undeformed reference frame to the twisted, curved, undeformed frame; $C^{B a}$ is the rotation matrix from the untwisted, uncurved, undeformed frame to the twisted, curved, deformed frame; and $U$ is the displacement of the twisted, curved, deformed frame with respect to twisted, curved, undeformed frame.

The first order discretized form of the above equations in the space domain can be represented as [3]:

$$
\begin{aligned}
U_{n+1} & =U_{n}+\left(C^{l r} C^{B a_{n}}\right)^{T}\left(\gamma+e_{1}\right) \Delta x_{n}-\left(C^{l r} C^{b a_{n}}\right)^{T} e_{1} \Delta x_{n} \\
C^{B a_{n+1}} & =\left(\frac{I_{3 \times 3}}{\Delta x_{n}}+\frac{\widetilde{k+\kappa}}{2}\right)^{-1}\left(\frac{I_{3 \times 3}}{\Delta x_{n}}-\frac{\widetilde{k+\kappa}}{2}\right) C^{B a_{n}} \\
C^{b a_{n+1}} & =\left(\frac{I_{3 \times 3}}{\Delta x_{n}}+\frac{\widetilde{k}}{2}\right)^{-1}\left(\frac{I_{3 \times 3}}{\Delta x_{n}}-\frac{\widetilde{k}}{2}\right) C^{b a_{n}}
\end{aligned}
$$

where $\Delta x_{n}$ is the length of each element and $C^{l r}$ is the element right-to-left rotation matrix which in this case is equal to identity matrix.

It is noted that the zero displacement and identity rotation matrices will be obtained when strain measures $\gamma$ and $\kappa$ vanish. Since $\gamma$ and $\kappa$ are related to $F, M$, $F^{a c t}$ and $M^{\text {act }}$ through constitutive relation of Eq. 3.9, repeated below, zero blade displacement is achieved when $F+F^{a c t}$ and $M+M^{\text {act }}$ vanish.

$$
\left\{\begin{array}{l}
\gamma \\
\kappa
\end{array}\right\}=\left[\begin{array}{cc}
R & S \\
S^{T} & T
\end{array}\right]\left\{\begin{array}{c}
F+F^{a c t} \\
M+M^{a c t}
\end{array}\right\}
$$

Forcing all three elements of the internal force, $F+F^{a c t}$, and internal moment, $M+M^{\text {act }}$, to vanish is equivalent to forcing displacements in all three directions to be zero. To do so, large control signals implying large actuation forces and moments are required. In addition, the objective of the BSP controller is to decrease the flapwise 
bending displacement of the blade, and the lateral (lead-lag) and the directional (axial) displacements are not of concern. Therefore, only elements of the internal forces and moments that have significant contributions to the flapping displacement of the blade are considered in the controller design. These elements are the vertical force $F_{3}+F_{3}^{a c t}$ and the bending moment $M_{2}+M_{2}^{\text {act }}$. Hence, the desired (zero) flapwise displacement can be obtained when $F_{3}+F_{3}^{a c t}$ and $M_{2}+M_{2}^{a c t}$ and their time derivatives vanish, i.e.,

$$
\begin{aligned}
& 0=F_{3}^{d}+F_{3}^{a c t^{d}}=\dot{F}_{3}^{d}+\dot{F}_{3}^{a c t^{d}} \\
& 0=M_{2}^{d}+M_{2}^{a c t^{d}}=\dot{M}_{2}^{d}+\dot{M}_{2}^{a c t^{d}}
\end{aligned}
$$

where indices 2 and 3 represent the second and the third elements of the associated vectors, respectively.

Eqs. 4.30 and 4.31 provide the necessary conditions to obtain the desired trajectory. Based on these conditions, the following desired values are considered:

- $F^{a c t^{d}}=0$ and $M^{a c t^{d}}=0$. This is because it is not desired to apply any actuation efforts to the system when it is in its desired condition. As a result, from Eqs. 4.30 and 4.31 one can conclude that $F_{3}^{d}=0$ and $M_{2}^{d}=0$.

- $F_{1}^{d}=F_{1}, F_{2}^{d}=F_{2}, M_{1}^{d}=M_{1}$, and $M_{3}^{d}=M_{3}$. This is because for the BSP control, the displacements in the other two directions are not important. Hence, the internal forces and moments affecting those displacements do not need to be controlled.

- $u_{1}^{d}=0$ and $u_{2}^{d}=0$. Control designers prefer minimum control effort when the controlled system is in its stable working condition. Therefore, it is decided to choose zero control values for the system in its desired trajectory. As a result, from Eqs. 3.15 and 3.16 one can conclude that $\dot{F}^{a c t}{ }^{d}=0$ and $\dot{M}^{a c t}=0$. Then 
from Eqs. 4.30 and $4.31, \dot{F}_{3}^{d}=0, \dot{M}_{2}^{d}=0$.

- $V^{d}=V, \Omega^{d}=\Omega, \lambda^{d}=\lambda$, and $g^{d}=g$. This is because there is no direct control on the variables $V, \Omega, \lambda$ and $g$. Therefore, the desired value of these variables are assumed to be equal to their actual value. It should be noted that although there is no direct control on these variables, they are indirectly controlled through controlling the internal forces and moments.

Replacing the above values in the power equations (Eqs. 4.8-4.13), the following deviated powers can be obtained:

$$
\begin{aligned}
& P^{a e r^{*}}=0 \\
& P^{d a m^{*}}=0 \\
& P^{g r^{*}}=0 \\
& P^{a c t^{*}}=\int_{0}^{L}\left[F_{3}^{a c t}{ }^{T}\left(R_{3}\left(\dot{F}+\dot{F}^{a c t}\right)+S_{3}\left(\dot{M}+\dot{M}^{a c t}{ }^{T}\right)\right)\right. \\
& \left.+M_{2}^{a c t}\left(S_{2}^{T}\left(\dot{F}+\dot{F}^{a c t}\right)+T_{2}\left(\dot{M}+\dot{M}^{a c t}{ }^{T}\right)\right)\right] \mathrm{d} x \\
& P^{a w^{*}}=\int_{0}^{L}\left[\left(F^{a c t} R^{T}+M^{a c t} S^{T}\right)\left(\dot{F}^{a c t}-u_{1}\right)\right. \\
& \left.+\left(F^{a c t} S+M^{a c t} T^{T}\right)\left(\dot{M}^{a c t}-u_{2}\right)\right] \mathrm{d} x \\
& P^{\text {bou* }}=-F_{3}(0) V_{3}^{0}-M_{2}(0) \Omega_{2}^{0}
\end{aligned}
$$

Generally, the deviated dynamics in the BSP is a non-autonomous system. Because the boundary values due to the engagement or disengagement can be explicit functions of time. However, since the structural dynamics of the blade varies much faster than the boundary values, the deviated dynamics can be assumed to behave as an autonomous dynamical system in sequential time intervals. Therefore, the candidate Lypunov function of this autonomous system that is the total deviated energy 
in the blade can be obtained from the following equation:

$$
V=T^{*}+U^{s t^{*}}
$$

$T+U^{s t}$ is a measure of the total energy in the blade. However, $T^{*}+U^{s t^{*}}$ is the portion of the energy in the blade that corresponds to its flapwise elastic motion.

$V$ is a positive definite function because it is the summation of two positive kinetic and strain energies. Therefore, it satisfies the first condition of the Lyapunov stability theroem for autonomous systems. The only thing left is to find controllers $u_{1}$ and $u_{2}$ to satisfy the second condition, i.e.,

$$
\dot{V}=P^{e x t^{*}}+P^{g r^{*}}+P^{b o u^{*}}+P^{a c t^{*}}+P^{a w^{*}}+P^{d a m^{*}}<0
$$

Substituting Eqs. 4.32-4.37 in Eq. 4.39, the following inequality will be obtained:

$$
\begin{gathered}
\int_{0}^{L}\left[\left(F^{a c t} R^{T}+M^{a c t} S^{T}\right)\left(\dot{F}^{a c t}-u_{1}\right)+\left(F^{a c t}{ }^{T} S+M^{a c t} T^{T}\right)\left(\dot{M}^{a c t}-u_{2}\right)\right] \mathrm{d} x+ \\
\int_{0}^{L}\left[F_{3}^{a c t}\left(R_{3}\left(\dot{F}+\dot{F}^{a c t}\right)+S_{3}\left(\dot{M}+\dot{M}^{a c t}{ }^{T}\right)\right)+M_{2}^{a c t}\left(S_{2}^{T}\left(\dot{F}+\dot{F}^{a c t}\right)+\right.\right. \\
\left.\left.T_{2}\left(\dot{M}+\dot{M}^{a c t}\right)\right)\right] \mathrm{d} x-F_{3}(0) V_{3}^{0}-M_{2}(0) \Omega_{2}^{0}<0
\end{gathered}
$$

To satisfy the second condition for the Lypunov function, or to make the time rate of change of the candidate Lyaunov function negative definite, one needs to find the control signals $u_{1}$ and $u_{2}$ such that the above inequality is satisfied. This is equivalent 
to choosing a control design factor $\beta$ such that:

$$
\begin{aligned}
& \int_{0}^{L}\left[\left(F^{a c t} R^{T}+M^{a c t^{T}} S^{T}\right) u_{1}+\left(F^{a c t} T^{T}+M^{a c t} T^{T}\right) u_{2}\right] \mathrm{d} x=\beta \times\{ \\
& \int_{0}^{L}\left[\left(F^{a c t} R^{T}+M^{a c t^{T}} S^{T}\right) \dot{F}^{a c t}+\left(F^{a c t} S^{T}+M^{a c t} T^{T}\right) \dot{M}^{a c t}\right] \mathrm{d} x+ \\
& \int_{0}^{L}\left[F_{3}^{a c t}\left(R_{3}\left(\dot{F}+\dot{F}^{a c t}\right)+S_{3}\left(\dot{M}+\dot{M}^{a c t^{T}}\right)\right)+\right. \\
& \left.\left.M_{2}^{a c t}\left(S_{2}^{T}\left(\dot{F}+\dot{F}^{a c t}\right)+T_{2}\left(\dot{M}+\dot{M}^{a c t}\right)\right)\right] \mathrm{d} x-F_{3}(0) V_{3}^{0}-M_{2}(0) \Omega_{2}^{0}\right\}
\end{aligned}
$$

To satisfy condition (4.40), depending on the sign of the right hand side of Eq. 4.41, $\beta$ must be either larger or smaller than one. A positive right-hand side requires $\beta>1$, and a negative right-hand side requires $\beta<1$.

Eq. 4.41 provides the continuous control signals along the blade. For actual control implementation, discrete control signals are required. One method to obtain discrete signals is to discretize the blade along its span and assume constant average control signals for each discretized element. Based on Eqs. 3.13-3.16, the control signals are related to the time rate of change of voltage along the piezo fibres, $\dot{V}_{p}$, through piezoelectric field coefficient vectors. Identifying $\dot{V}_{p}$ as $v$, we have:

$$
\begin{gathered}
u_{1}=Y^{F} v \\
u_{2}=Y^{M} v
\end{gathered}
$$


Defining the following variables to simplify the notation

$$
\begin{aligned}
& X^{A}=\left[\left(F^{a c t} R^{T}+M^{a c t} S^{T}\right) Y^{F}+\left(F^{a c t}{ }^{T} S+M^{a c t} T^{T}\right) Y^{M}\right] \\
& Y^{A}=\left[\left(F^{a c t} R^{T}+M^{a c t} S^{T}\right) \dot{F}^{a c t}+\left(F^{a c t}{ }^{T} S+M^{a c t} T^{T}\right) \dot{M}^{a c t}\right]+ \\
& {\left[F_{3}^{a c t^{T}}\left(R_{3}\left(\dot{F}+\dot{F}^{a c t}\right)+S_{3}\left(\dot{M}+\dot{M}^{a c t}\right)\right)+\right.} \\
& \left.M_{2}^{a c t}\left(S_{2}^{T}\left(\dot{F}+\dot{F}^{a c t}\right)+T_{2}\left(\dot{M}+\dot{M}^{a c t}\right)\right)\right]
\end{aligned}
$$

and substituting Eqs. 4.42-4.45 into Eq. 4.41, the following can be obtained:

$$
\int_{0}^{L} X^{A} v \mathrm{~d} x=\beta \times\left\{\int_{0}^{L} Y^{A} \mathrm{~d} x+P^{b o u^{*}}\right\}
$$

Using first order discretization with number of elements ne, the average $v$ signals in each element can be obtained from:

$$
\bar{v}_{i}=\frac{\int_{(i-1) \frac{L}{n e}}^{i \frac{L}{n e}} X^{A} v \mathrm{~d} x}{\int_{(i-1) \frac{L}{n e}}^{i \frac{L}{n e}} X^{A} \mathrm{~d} x}
$$

where subscript $i$ shows the element number. Substituting Eq. 4.46 into Eq. 4.47, and integrating along each element length, one can obtain:

$$
\bar{v}_{i}=\frac{\beta_{i}\left\{\int_{(i-1) \frac{L}{n e}}^{i \frac{L}{n e}} Y^{A} \mathrm{~d} x+P^{b o u^{*}} / n e\right\}}{\int_{(i-1) \frac{L}{n e}}^{i \frac{L}{n e}} X^{A} \mathrm{~d} x}
$$

where $\beta_{i}$ is a controller designing factor that can be tuned separately for each element, based on a tuning criterion such as requiring the minimum control effort (active piezo fibres voltages) along the blade. As can be seen, to design the controller, a uniform distribution of boundary power along the blade is assumed. Different distributions 
along with different constant factors $\beta_{i}$ can be used to obtain different control signals along the blade.

The above controller stabilizes the deviated (error) dynamical system. Since the stability and the trajectory following problems are dual, this stabilization corresponds to trajectory following in the real system. In the following chapters, after applying the designed controller, the PDEs are reduced to ODEs through the Galerkin Spectral Method and the resultant ODEs are solved numerically. 


\section{Chapter 5}

\section{Galerkin Spectral Discretization}

In this chapter, the governing equations derived in the previous chapter are discretized using the Galerkin Spectral Discretization Method. After discretization and removing the spatial parts, the remaining ODEs can be solved using established numerical methods such as Runge-Kutta. This chapter begins by introducing the Galerkin Spectral Method. Then the selected weighting and comparison functions are explained and justified. Following that, the PDEs are reduced to ODEs, and the resulting ODEs are expressed in a tensor form using Einstein tensor notation. Finally these ODEs are simplified to the case with constant coefficients for variables. This simplification is achieved using constant cross sectional properties, as well as aerodynamic parameters along the blade.

\subsection{The Galerkin Method Concept}

Except for special cases, most Partial Differential Equations (PDEs) are not solvable analytically. Numerical methods for solving PDEs are categorized into local and global methods. The Finite Difference Method (FDM) and Finite Element Method (FEM) are based on local parameters, whereas the Spectral Methods are based on global ones. In structural analysis problems, the finite element method is the most 
commonly used technique for discretization of PDEs in the space domain reducing them to Ordinary Differential Equations (ODEs) in the time domain. FEMs are well-suited to 2-D and 3-D problems with complex geometries. Spectral Methods, on the other hand, can provide better accuracy for 1-D problems at the expense of geometry flexibility [35].

In this research, since all in-plane and out-of-plane deformation effects are captured in the blade cross sectional flexibility/stiffness matrices and a compact set of ordinary differential equations are required for controller design purposes, the conventional Galerkin Spectral Discretization Method is adopted. This method has previously been used for discretization of a helicopter rotor blade for control purposes [25]. As stated earlier, the main difference between Galerkin Spectral Method and the FEM approach is that unlike in FEM, in the Galerkin method discretization is performed globally using a predefined set of global spatial functions, called comparison (trial) functions. In the following paragraphs, the concept of the Galerkin Spectral Discretization Method is further elaborated.

Assuming the solution of the partial differential equation $f\left(w(x, t), w(\dot{x}, t), w^{\prime}(x, t), x, t\right)=0$ is dependent on time and space, an approximate solution $w(x, t)$ of it can be represented as the sum of products of two sets of functions: one set of space functions $\Phi_{i}(x)$ called comparison functions or generalized modes, and one set of time functions $q_{i}(t)$ called generalized coordinates, i.e.,

$$
w(x, t)=\sum_{i=1}^{n c} \Phi_{i}(x) q_{i}(t)
$$


where $n c$ denotes the number of comparison functions used in the summation. Replacing (5.1) in the PDE equation results in:

$$
\begin{gathered}
f\left(w(x, t), \dot{w}(x, t), w^{\prime}(x, t), x, t\right)=0 \Longrightarrow \\
f_{i}\left(\Phi_{i}(x), \Phi_{i}^{\prime}(x), q_{i}(t), \dot{q}(t), x, t\right) \approx 0 \\
, i=1,2, \ldots, n c
\end{gathered}
$$

The right-hand equation in Eq. 5.2 is not exactly equal to zero. However, weighting (sometimes called test) functions $\Xi_{l}(x)$ can be found such that the integration of the product of these weighting functions and the above functions $f_{i}$ along the domain becomes zero, i.e.,

$$
\int_{0}^{L}\left[\Xi_{l}(x) f\left(\Phi_{i}(x), \Phi_{i}^{\prime}(x), q_{i}(t), \dot{q}(t), x, t\right)\right] \mathrm{d} x=0, \quad l, i=1,2, \ldots, n c
$$

The final set of Eqs. 5.3 are only time differential equations as the integration eliminates the space dependent part. Theoretically, if an infinite number of comparison functions are chosen for discretization $(n c \longrightarrow \infty)$, the Galerkin Spectral Method solves the original partial differential equation exactly.

\subsection{Weighting Functions}

The quality of the Galerkin discretized model relies on the proper choice of comparison functions $\Phi_{i}(x)$ and weighting functions $\Xi_{l}(x)$ [36]. Since, the weighting functions used in the previous chapter satisfy the law of conservation of energy, the same weighting functions are employed for discretization. These weighting functions can be approximated as the sum of product of their spatial functions and temporal functions. However, as can be seen in Eq. 5.3, only the spatial part of those weighting functions are required for discretization.

It can be shown that using the spatial part does not affect the law of conservation 
of energy. For example, if the blade first kinematic equation, Eq. 3.3, is premultiplied by the spatial part of the weighting function $F^{T}(x, t)+F^{a c t}{ }^{T}(x, t)$, one obtains:

$$
\int_{0}^{L}\left\{\left[\overline{F_{l}^{T}}(x)+\overline{F_{l}^{\text {act }}}(x)\right]\left[\dot{\gamma}-V^{\prime}-(\widetilde{k+\kappa}) V-\left(\widetilde{e_{1}+\gamma}\right) \Omega\right]\right\} \mathrm{d} x=0
$$

where the overline indicates the spatial part of the weighting function. If Eq. 5.4 is satisfied, then the following equation will be satisfied, as well:

$$
\int_{0}^{L}\{\underbrace{\sum_{l=1}^{n c}\left[\left(\overline{F_{l}^{T}}(x)+\overline{F_{l}^{a c t} t^{T}}(x)\right) q_{l}(t)\right]}_{\approx F^{T}+F^{a c t} t^{T}}\left[\dot{\gamma}-V^{\prime}-(\widetilde{k+\kappa)})-\left(\widetilde{e_{1}+\gamma}\right) \Omega\right]\} \mathrm{d} x=0
$$

In a similar manner, it can be shown that instead of using previously-defined weighting functions, the spatial part of those weighting functions can be used as weighting functions for discretization of all governing equations.

\subsection{Comparison Functions}

To apply the Galerkin Spectral Method, each variable that is a function of both time and space has to be approximated as a sum of product of one spatial function (comparison function) and one temporal function. For example, the approximation of the state variable $F$ is formulated as:

$$
F(x, t)=\sum_{i=1}^{n c} \bar{F}_{i}(x) \underline{F}_{i}(t), \quad \bar{F}_{i}(x)=\left[\begin{array}{ccc}
\bar{F}_{1 i} & 0 & 0 \\
0 & \bar{F}_{2 i} & 0 \\
0 & 0 & \bar{F}_{3 i}
\end{array}\right], \quad \underline{F}_{i}(t)=\left\{\begin{array}{l}
\underline{F}_{1 i} \\
\underline{F}_{2 i} \\
\\
\underline{F}_{3 i}
\end{array}\right\}
$$


where subscripts 1 to 3 are the direction indices for the state vector $F$. All other state variables $M, F^{\text {act }}, M^{\text {act }}, V, \Omega, g$, and $\lambda$, present in the equations, are approximated in a similar manner. This approximation is outlined in Appendix C.

For ease of handling all eight temporal functions, a generalized temporal function $q_{i}(t)$ (generalized coordinate), containing all temporal functions and a generalized magnitude temporal function $\left|q_{i}(t)\right|$ containing the magnitude (absolute value) of all temporal functions are introduced such that:

$$
\begin{aligned}
& q_{i}=\left\{\underline{V}_{i}, \underline{\Omega}_{i}, \underline{F_{i}}, \underline{M}_{i}, \underline{\lambda}_{i}, \underline{g}_{i}, \underline{F}_{i}^{a c t}, \underline{M}_{i}^{a c t}\right\}_{(24 \times 1)}^{T} \\
& \left|q_{i}\right|=\left\{\left|\underline{V}_{i}\right|,\left|\underline{\Omega}_{i}\right|,\left|\underline{F}_{i}\right|,\left|\underline{M}_{i}\right|,\left|\underline{\lambda}_{i}\right|,\left|\underline{g}_{i}\right|,\left|\underline{F}_{i}^{a c t}\right|,\left|\underline{M}_{i}^{a c t}\right|\right\}_{(8 \times 1)}^{T}
\end{aligned}
$$

Using this generalized temporal function, Eq. 5.6 can now be rewritten as:

$$
F(x, t)=\sum_{i=1}^{n c} \Phi_{i}^{F}(x) q_{i}(t), \quad \Phi_{i}^{F}(x)=\left[0,0, \bar{F}_{i}, 0,0,0,0\right]_{(3 \times 24)}
$$

where $\Phi_{i}^{F}(x)$ can be called a generalized mode associated with the variable $F(x, t)$. Approximation of other state variables with the generalized temporal function $q_{i}(t)$ along with matrices and vectors that are used to derive the final ODEs are given in Appendix C.

Comparison functions such as $\Phi_{i}^{F}(x)$ in the above equation, generally can be divided into two major groups: periodic comparison functions and non-periodic comparison functions.

Periodic comparison functions such as Fourier series are suitable when the solution and the boundary conditions are periodic. Periodic solution means that, at every instant of time, the solved variable is periodically distributed along the domain. If the problem is not periodic, using the Fourier series will convert it to a periodic problem with discontinuity at one boundary. This discontinuity and the overshoot that can be 
seen in its neighbourhood as $n c \rightarrow \infty$ is referred to as the Gibbs phenomenon in the literature [36]. Another constraint with periodic comparison functions is their need to have an infinitely differentiable exact solution with homogeneous boundary conditions. Their rate of convergence is typically $O\left(n c^{-2}\right)$ except close to the boundary, where it is $O\left(n c^{-1}\right)$ [36].

Legendre and Chebyshev polynomials are two main non-periodic comparison functions. Legendre polynomials are orthogonal in the range $-1 \leq x \leq-1$ with respect to weight function $(w(x)=1)$. This would result in a sparse mass matrix ( $A_{l i}$ in the following discretized equations) with no off-diagonal elements and a well-conditioned solution. In addition, they are easy to integrate and implement. However, the global convergence rate of Legendre polynomials is $O\left(n c^{-1}\right)$ except close to boundaries where it drops to $O\left(n c^{-1 / 2}\right)$. This low convergence rate is their main disadvantage.

Chebyshev polynomials are orthogonal in the range $-1 \leq x \leq-1$ with respect to weight function $\left(w(x)=\left(1-x^{2}\right)^{1 / 2}\right)$ [35]. This means that application of Chebyshev polynomials in conventional Galerkin Spectral formulations would produce offdiagonal elements in the mass matrix. However, they have the same convergence rate as the Fourier series functions.

If identical polynomials are used for both comparison and weighting functions, the Galerkin discretization method is called TraditionalGalerkin or Conventional Galerkin. This method is also known as the Bubnov-Galerkin method in some literature [37]. In the Modern Galerkin or Generalized Galerkin method, which is sometimes called Petrov-Galerkin method, to avoid possible solution instability problems that the traditional method suffers from, weighting functions are chosen to be different from the comparison functions [36]. As an example, if one chooses Chebyshev polynomials as comparison functions and Chebyshev polynomials divided by $\left(1-x^{2}\right)^{1 / 2}$ as weighting functions, in addition to avoiding the possible instability in the solution, they can take advantage of the weighted orthogonality of 
Chebyshev polynomials.

In this research, since the boundary conditions and the solution are not periodic, and in order to take advantage of sparsity of the resulting mass matrix and subsequent ease in implementation and solution, Legendre polynomials are employed as comparison functions. In addition, the same polynomials are chosen for the spatial part of the weighting functions.

Regular Legendre functions are orthogonal in the domain $-1 \leq x \leq 1$. However, for the helicopter blade, the domain of interest is from the blade root to its tip, i.e., $0 \leq x \leq L$. Therefore, normalized shifted Legendre functions have to be used for discretization. These functions can be obtained by replacing $x$ by $\frac{2 x-1}{L}$ in regular Legendre functions. The shifted polynomials have the same properties in the domain $0 \leq \frac{x}{L} \leq 1$ as the regular ones in $-1 \leq x \leq 1$. Orthogonality of the normalized shifted Legendre polynomials can be shown as:

$$
\int_{0}^{L} P_{l}(x / L) P_{k}(x / L) \mathrm{d} x=\frac{1}{2(l-1)+1} \delta_{l k}
$$

where $\delta_{l k}$ denotes the Kronecker delta, equal to 1 if $l=k$ and 0 otherwise. These polynomials can be obtained using the following Rodrigues' formula [38]:

$$
P_{l}(x / L)=\frac{1}{n !} \frac{d^{l}}{\mathrm{~d} x^{l} / L}\left[\left(x^{2} / L^{2}-x / L\right)^{l}\right]
$$

The first five normalized Legendre polynomials are shown in Fig. 5.1. In the following sections, first the discretized state and output functions are obtained using any arbitrary comparison and weighting (spatial part) functions. Then, using Legendre polynomials for comparison functions and the spatial part of the weighting functions, equations will be derived for a special case in which structural and inertial properties of the blade are constant along the blade. 


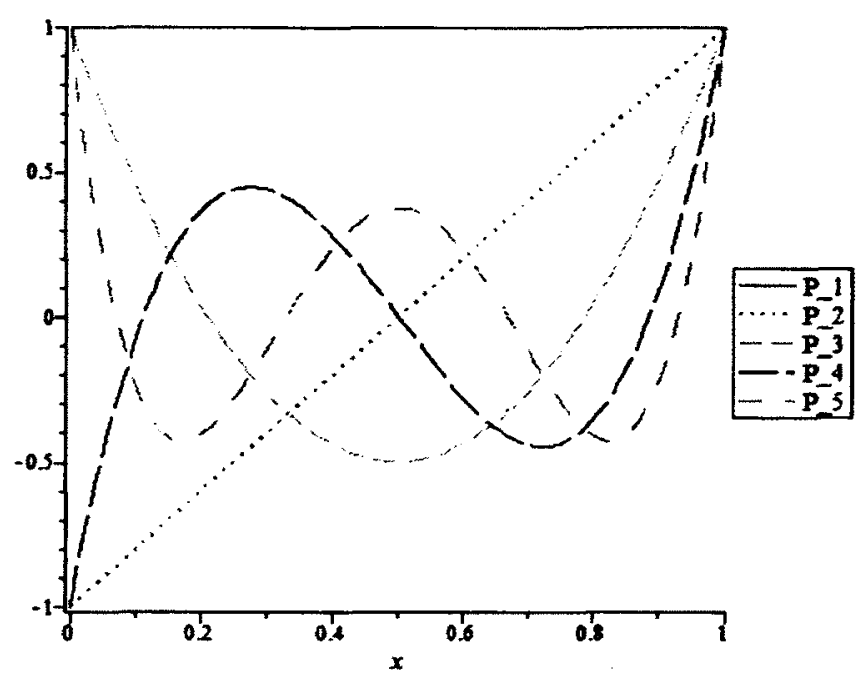

Figure 5.1: First five Legendre Polynomials.

\subsection{State Equations}

Using the continuous form of the control signals along the blade to close the control loop (substituting Eq. 4.41 in Eq. 4.16), and applying comparison functions for discretization, the discretized closed-loop state space form of Eq. 4.16 becomes:

$$
\begin{aligned}
& 0=\int_{0}^{L}\left\{\Phi_{l}^{V^{T}}\left[G \Phi_{i}^{V}+Q \Phi_{i}^{\Omega}-J \Phi_{i}^{F}-J \Phi_{i}^{F^{a c t}}-N \Phi_{i}^{M}-N \Phi_{i}^{M^{a c t}}\right] \dot{q}_{i}+\right. \\
& \Phi_{l}^{V^{T}}\left[-\Phi_{i}^{F^{\prime}}-\tilde{k} \Phi_{i}^{F}-G g_{m} \Phi_{i}^{g}\right] q_{i}-\Phi_{l}^{V^{T}}\left[f^{a}\right]-\Phi_{l}^{\Omega^{T}}\left[m^{a}\right]+ \\
& \Phi_{l}^{V^{T}}\left[\Phi_{i}^{\tilde{\Omega}}\left(G \Phi_{j}^{V}+Q \Phi_{j}^{\Omega}\right)-\left(\widetilde{S^{T} \Phi_{i}^{F}}+\widetilde{S^{T} \Phi_{i}^{F^{a c t}}}+\widetilde{T \Phi_{i}^{M}}+\widetilde{T \Phi_{i}^{M^{a c t}}}\right) \Phi_{j}^{F}\right] \\
& q_{i} q_{j}+\Phi_{l}^{\Omega^{T}}\left[Q^{T} \Phi_{i}^{V}+I \Phi_{i}^{\Omega}-B \Phi_{i}^{F}-B \Phi_{i}^{F^{a c t}}-W \Phi_{i}^{M}-W \Phi_{i}^{M^{\text {act }}}\right] \dot{q}_{i}+ \\
& \Phi_{l}^{\Omega^{T}}\left[-\Phi_{i}^{M^{\prime}}-\widetilde{e_{1}} \Phi_{i}^{F}-\tilde{k} \Phi_{i}^{M}-Q g_{m} \Phi_{i}^{g}\right] q_{i}+\Phi_{l}^{\Omega^{T}}\left[\Phi_{i}^{\tilde{\Omega}}\left(Q^{T} \Phi_{j}^{V}+I \Phi_{j}^{\Omega}\right)+\right. \\
& \Phi_{i}^{\tilde{V}}\left(G \Phi_{j}^{V}+Q \Phi_{j}^{\Omega}\right)-\left(\widetilde{S^{T} \Phi_{i}^{F}}+\widetilde{S^{T} \Phi_{i}^{F^{a c t}}}+\widetilde{T \Phi_{i}^{M}}+T \widetilde{\Phi_{i}^{M^{a c t}}}\right) \Phi_{j}^{M}- \\
& \left.\left(\widetilde{R \Phi_{i}^{F}}+R \widetilde{R \Phi_{i}^{F^{a c t}}}+\widetilde{S \Phi_{i}^{M}}+S \widetilde{\Phi_{i}^{M a c t}}\right) \Phi_{j}^{F}\right] q_{i} q_{j}+\Phi_{l}^{F^{T}}\left[R \Phi_{i}^{F}+R \Phi_{i}^{F^{a c t}}+\right. \\
& \left.S \Phi_{i}^{M}+S \Phi_{i}^{M^{a c t}}\right] \dot{q}_{i}+\Phi_{l}^{F^{a c t} T}\left[R \Phi_{i}^{F}+R \Phi_{i}^{F^{a c t}}+S \Phi_{i}^{M}+S \Phi_{i}^{M^{a c t}}\right] \dot{q}_{i}+
\end{aligned}
$$




$$
\begin{aligned}
& \Phi_{l}^{F^{T}}\left[-\Phi_{i}^{V^{\prime}}-\widetilde{e_{1}} \Phi_{i}^{\Omega}-\widetilde{k} \Phi_{i}^{V}\right] q_{i}+\Phi_{l}^{F^{a c t} T}\left[-\Phi_{i}^{V^{\prime}}-\widetilde{e_{1}} \Phi_{i}^{\Omega}-\widetilde{k} \Phi_{i}^{V}\right] q_{i}+ \\
& \Phi_{l}^{F^{T}}\left[-\left(\widetilde{S^{T} \Phi_{i}^{F}}+\widetilde{S^{T} \Phi_{i}^{F^{a c t}}}+\widetilde{T \Phi_{i}^{M}}+\widetilde{T \Phi_{i}^{M^{a c t}}}\right) \Phi_{j}^{V}-\left(\widetilde{R \Phi_{i}^{F}}+\widetilde{R \Phi_{i}^{F^{a c t}}}+\right.\right. \\
& \left.\left.\widetilde{S \Phi_{i}^{M}}+S \widetilde{S \Phi_{i}^{M^{a c t}}}\right) \Phi_{j}^{\Omega}\right] q_{i} q_{j}+\Phi_{l}^{F^{a c t} T}\left[-\left(\widetilde{S^{T} \Phi_{i}^{F}}+\widetilde{S^{T} \Phi_{i}^{F^{a c t}}}+\widetilde{T \Phi_{i}^{M}}+\right.\right. \\
& \left.\left.T \widetilde{\Phi_{i}^{M^{a c t}}}\right) \Phi_{j}^{V}-\left(\widetilde{R \Phi_{i}^{F}}+\widetilde{R \Phi_{i}^{F^{a c t}}}+\widetilde{S \Phi_{i}^{M}}+S \widetilde{S \Phi_{i}^{M^{a c t}}}\right) \Phi_{j}^{\Omega}\right] q_{i} q_{j}+ \\
& \Phi_{l}^{M^{T}}\left[S^{T} \Phi_{i}^{F}+S^{T} \Phi_{i}^{F^{a c t}}+T \Phi_{i}^{M}+T \Phi_{i}^{M^{a c t}}\right] \dot{q}_{i}+\Phi_{l}^{M^{a c t} T}\left[S^{T} \Phi_{i}^{F}+\right. \\
& \left.S^{T} \Phi_{i}^{F^{a c t}}+T \Phi_{i}^{M}+T \Phi_{i}^{M^{a c t}}\right] \dot{q}_{i}+\Phi_{l}^{M^{T}}\left[-\Phi_{i}^{\Omega^{\prime}}-\tilde{k} \Phi_{i}^{\Omega}\right] q_{i}+\Phi_{l}^{M^{a c t} T} \\
& {\left[-\Phi_{i}^{\Omega^{\prime}}-\tilde{k} \Phi_{i}^{\Omega}\right] q_{i}+\Phi_{i}^{M^{T}}\left[-\left(\widetilde{S^{T} \Phi_{i}^{F}}+\widetilde{S^{T} \Phi_{i}^{F^{a c t}}}+\widetilde{T \Phi_{i}^{M}}+\widetilde{T \Phi_{i}^{M^{a c t}}}\right)\right.} \\
& \left.\Phi_{j}^{\Omega}\right] q_{i} q_{j}+\Phi_{i}^{M^{a c t} T}\left[-\left(\widetilde{S^{T} \Phi_{i}^{F}}+\widetilde{S^{T} \Phi_{i}^{F^{a c t}}}+\widetilde{T \Phi_{i}^{M}}+\widetilde{T \Phi_{i}^{M^{a c t}}}\right) \Phi_{j}^{\Omega}\right] q_{i} q_{j}+ \\
& \Phi_{l}^{V^{T}}\left[-G g_{m} x \Phi_{i}^{g^{\prime}}-G g_{m} x \widetilde{k} \Phi_{i}^{g}\right] q_{i}+\Phi_{l}^{\Omega^{T}}\left[-Q g_{m} x \Phi_{i}^{g^{\prime}}-Q g_{m} x \tilde{k} \Phi_{i}^{g}\right] q_{i}+ \\
& \Phi_{l}^{V^{T}}\left[\left(-G g_{m} x \widetilde{S^{T} \Phi_{i}^{F}}-G g_{m} x S^{T} \widetilde{\Phi_{i}^{F^{a c t}}}-G g_{m} x \widetilde{T \Phi_{i}^{M}}-G g_{m} x T \widetilde{\Phi_{i}^{M^{a c t}}}\right) \Phi_{j}^{g}\right] \\
& q_{i} q_{j}+\Phi_{l}^{\Omega^{T}}\left[\left(-Q g_{m} x \widetilde{S^{T} \Phi_{i}^{F}}-Q g_{m} x \widetilde{S^{T} \Phi_{i}^{F^{a c t}}}-Q x \widetilde{T \Phi_{i}^{M}}-Q g_{m} x T \widetilde{\Phi_{i}^{M^{a c t}}}\right)\right. \\
& \left.\Phi_{j}^{g}\right] q_{i} q_{j}+\Phi_{l}^{g^{T}}\left[-G g_{m} x-Q g_{m}\right] \Phi_{i}^{g} \dot{q}_{i}+\Phi_{l}^{g^{T}}\left[\left(-G g_{m} x-Q g_{m}\right) \Phi_{i}^{\tilde{\Omega}}\right] q_{i} q_{j}- \\
& \Phi_{l}^{\left|V^{T} G g_{m}\right|} \Phi_{i}^{|g|}\left|q_{i}\right|-\Phi_{l}^{\left|\Omega^{T} Q g_{m}\right|} \Phi_{i}^{|g|}\left|q_{i}\right|+\Phi_{l}^{\left|V^{T} G g_{m}\right|}+\Phi_{l}^{\left|\Omega^{T} Q g_{m}\right|}+ \\
& {\left[e^{\lambda^{T}} e_{2}^{\lambda} \Phi_{l}^{\lambda^{T}} X_{f 2 \lambda \lambda}+e^{V^{T}} e_{2}^{V} \Phi_{l}^{V^{T}} X_{f 2 V \lambda}+e^{\Omega^{T}} e_{2}^{\Omega} \Phi_{l}^{\Omega^{T}} X_{f 2 \Omega \lambda}+e^{V^{T}} e_{3}^{V} \Phi_{l}^{V^{T}}\right.} \\
& X_{f 3 V \lambda}+e^{\lambda^{T}} e_{1}^{\lambda} \Phi_{l}^{\lambda^{T}} X_{m 1 \lambda \lambda} / a b+e^{V^{T}} e_{1}^{V} \Phi_{l}^{V^{T}} X_{m 1 V \lambda} / a b+e^{\Omega^{T}} e_{1}^{\Omega} \Phi_{l}^{\Omega^{T}} \\
& \left.X_{m 1 \Omega \lambda} / a b\right]\left[\left(A_{i n f} \Phi_{i}^{\lambda}+C_{i n f} \Phi_{i}^{V}+D_{i n f} \Phi_{i}^{\Omega}\right) \dot{q}_{i}+\left(e_{1}^{V} \Phi_{i}^{V^{T}} X_{i n f 1 V \lambda} \Phi_{j}^{\lambda}+\right.\right. \\
& \left.\left.e_{1}^{V} \Phi_{i}^{V^{T}} X_{i n f 2 V \lambda} \Phi_{j}^{\lambda}+e_{3}^{V} \Phi_{i}^{V^{T}} X_{i n f 3 V \lambda} \Phi_{j}^{\lambda}\right) q_{i} q_{j}\right]+\Phi_{i}^{F^{a c t T}}\left[R^{T} \Phi_{i}^{F^{a c t}}+\right. \\
& \left.S \Phi_{i}^{M^{a c t}}\right] \dot{q}_{i}+\Phi_{l}^{M^{a c t} T}\left[S^{T} \Phi_{i}^{F^{a c t}}+T^{T} \Phi_{i}^{M^{a c t}}\right] \dot{q}_{i}-\Phi_{l}^{F^{a c t} T}\left[R^{T} \Phi_{i}^{F^{a c t}}+\right. \\
& \left.S \Phi_{i}^{M^{a c t}}\right] \beta \dot{q}_{i}-\Phi_{l}^{M^{a c t} T}\left[S^{T} \Phi_{i}^{F^{a c t}}+T^{T} \Phi_{i}^{M^{a c t}}\right] \beta \dot{q}_{i}-\Phi_{l_{3}}^{F^{a c t} T}\left[R_{3} \Phi_{i}^{F}+\right. \\
& \left.R_{3} \Phi_{i}^{F^{a c t}}\right] \beta \dot{q}_{i}-\Phi_{l_{3}}^{F^{a c t} T}\left[S_{3} \Phi_{i}^{M}+S_{3} \Phi_{i}^{M^{a c t}}\right] \beta \dot{q}_{i}-\Phi_{l_{2}}^{M^{a c t} T}\left[S_{2}^{T} \Phi_{i}^{F}+\right.
\end{aligned}
$$




$$
\begin{aligned}
& \left.\left.S_{2}^{T} \Phi_{i}^{F^{a c t}}\right] \beta \dot{q}_{i}-\Phi_{l_{2}}^{M^{a c t} T}\left[T_{2} \Phi_{i}^{M}+T_{2} \Phi_{i}^{M^{a c t}}\right] \beta \dot{q}_{i}\right\} \mathrm{d} x+ \\
& \Phi_{l}^{V^{T}}(L)\left[\Phi_{i}^{F}(L)\right] q_{i}-\Phi_{l}^{V^{T}}(L)\left\{F^{L}\right\}+\Phi_{l}^{\Omega^{T}}(L)\left[\Phi_{i}^{M}(L)\right] q_{i}-\Phi_{l}^{\Omega^{T}}(L)\left\{M^{L}\right\}- \\
& \Phi_{l}^{F^{T}}(0)\left[\Phi_{i}^{V}(0)\right] q_{i}-\Phi_{l}^{F^{a c t}}(0)\left[\Phi_{i}^{V}(0)\right] q_{i}+\Phi_{l}^{F^{T}}(0)\left\{V^{0}\right\}+\Phi_{l}^{F^{a c t}}(0)\left\{V^{0}\right\}- \\
& \Phi_{l}^{M^{T}}(0)\left[\Phi_{i}^{\Omega}(0)\right] q_{i}-\Phi_{l}^{M^{a c t}}(0)\left[\Phi_{i}^{\Omega}(0)\right] q_{i}+\Phi_{l}^{M^{T}}(0)\left\{\Omega^{0}\right\}+\Phi_{l}^{M^{a c t}}(0)\left\{\Omega^{0}\right\}- \\
& \Phi_{l}^{V^{T}}(0)\left[G L g_{m} \Phi_{i}^{g}(0)\right] q_{i}-\Phi_{l}^{\Omega^{T}}(0)\left[Q L g_{m} \Phi_{i}^{g}(0)\right] q_{i}+\Phi_{l}^{V^{T}}(0) G L g_{m}\left\{g^{0}\right\}+ \\
& \Phi_{l}^{\Omega^{T}}(0) Q L g_{m}\left\{g^{0}\right\}+\Phi_{l}^{F_{3}}(0) \beta V_{3}^{0}+\Phi_{l}^{M_{2}}(0) \beta \Omega_{2}^{0}
\end{aligned}
$$

To represent the above equation in a simple form, Einstein tensor notation is employed [36]. By adding the aerodynamic loads, Eq. 5.12 can be summarized as the following tensor equation:

$$
A_{l i} \dot{q}_{i}+B_{l i} q_{i}+C_{l i j} q_{i} q_{j}+E_{l i / 3}\left|q_{i}\right|+F_{l}=0
$$

where $A_{l i}$ and $B_{l i}$ are $(24 \times n c, 24 \times n c)$ two-dimensional tensors (matrices), $C_{l i j}$ is a $(24 \times n c, 24 \times n c, 24 \times n c)$ three-dimensional tensor, $E_{l i}$ is a $(24 \times n c, 8 \times n c)$ twodimensional tensor (matrix), and $F_{l}$ is $(24 \times n c, 1)$ one-dimensional tensor (vector), and $n c$ is the number of comparison functions.

The tensors in Eq. 5.13 have the following components:

$$
\begin{aligned}
A_{l i} & =A_{l i}^{n o B C}+A_{l i}^{C O}+A_{l i}^{A E} \\
B_{l i} & =B_{l i}^{n o B C}+B_{l i}^{B C} \\
C_{l i j} & =C_{l i j}^{n o B C}+C_{l i j}^{A E} \\
E_{l i / 3} & =E_{l i / 3}^{n o B C} \\
F_{l} & =F_{l}^{n o B C}+F_{l}^{B C}+F_{l}^{C O}
\end{aligned}
$$

where superscripts no $B C, B C, C O$, and $A E$ indicate that the associated tensor is not 
due to boundary conditions, is due to boundary conditions, is due to the controller, and is due to aerodynamic forces and moments, respectively.

More explicitly, each of the tensor components in Eqs. 5.14-5.18 are identified as:

$$
\begin{aligned}
A_{l i}^{n o B C}=\int_{0}^{L}\{ & \Phi_{l}^{V^{T}}\left[G \Phi_{i}^{V}+Q \Phi_{i}^{\Omega}-J \Phi_{i}^{F}-J \Phi_{i}^{F^{a c t}}-N \Phi_{i}^{M}-N \Phi_{i}^{M^{a c t}}\right]+\Phi_{l}^{\Omega^{T}} \\
& {\left[Q^{T} \Phi_{i}^{V}+I \Phi_{i}^{\Omega}-B \Phi_{i}^{F}-B \Phi_{i}^{F^{a c t}}-W \Phi_{i}^{M}-W \Phi_{i}^{M^{a c t}}\right]+\Phi_{l}^{F^{T}} } \\
& {\left[R \Phi_{i}^{F}+R \Phi_{i}^{F^{a c t}}+S \Phi_{i}^{M}+S \Phi_{i}^{M^{a c t}}\right]+\Phi_{l}^{F^{a c t} T}\left[R \Phi_{i}^{F}+R \Phi_{i}^{F^{a c t}}+\right.} \\
& \left.S \Phi_{i}^{M}+S \Phi_{i}^{M^{a c t}}\right]+\Phi_{l}^{F^{a c t} T}\left[R^{T} \Phi_{i}^{F^{a c t}}+S \Phi_{i}^{M^{a c t}}\right]+\Phi_{l}^{M^{T}}\left[S^{T} \Phi_{i}^{F}+\right. \\
& \left.S^{T} \Phi_{i}^{F^{a c t}}+T \Phi_{i}^{M}+T \Phi_{i}^{M^{a c t}}\right]+\Phi_{l}^{M^{a c t} T}\left[S^{T} \Phi_{i}^{F}+S^{T} \Phi_{i}^{F^{a c t}}+T \Phi_{i}^{M}\right. \\
& \left.+T \Phi_{i}^{M^{a c t}}\right]+\Phi_{l}^{M^{a c t} T}\left[S^{T} \Phi_{i}^{F^{a c t}}+T^{T} \Phi_{i}^{M^{a c t}}\right]+\Phi_{l}^{g^{T}}\left[-G g_{m} x \Phi_{i}^{g}-\right. \\
& \left.Q g_{m} \Phi_{i}^{g}\right]+\left[e^{\lambda^{T}} e_{2}^{\lambda} \Phi_{l}^{\lambda^{T}} X_{f 2 \lambda \lambda}+e^{V^{T}} e_{2}^{V} \Phi_{l}^{V^{T}} X_{f 2 V \lambda}+e^{\Omega^{T}} e_{2}^{\Omega} \Phi_{l}^{\Omega^{T}} X_{f 2 \Omega \lambda}\right. \\
& +e^{V^{T}} e_{3}^{V} \Phi_{l}^{V^{T}} X_{f 3 V \lambda}+e^{\lambda^{T}} e_{1}^{\lambda} \Phi_{l}^{\lambda^{T}} X_{m 1 \lambda \lambda} / a b+e^{V^{T}} e_{1}^{V} \Phi_{l}^{V^{T}} X_{m 1 V \lambda} / a b \\
& \left.\left.+e^{\Omega^{T}} e_{1}^{\Omega} \Phi_{l}^{\Omega^{T}} X_{m 1 \Omega \lambda} / a b\right]\left[A_{i n f} \Phi_{i}^{\lambda}+C_{i n f} \Phi_{i}^{V}+D_{i n f} \Phi_{i}^{\Omega}\right]\right\} \mathrm{d} x \\
A_{l i}^{C O}=\int_{0}^{L}\{ & -\Phi_{l}^{F^{a c t T}}\left[R^{T} \Phi_{i}^{F^{a c t}}+S \Phi_{i}^{M^{a c t}}\right] \beta-\Phi_{l}^{M^{a c t} T}\left[S^{T} \Phi_{i}^{F^{a c t}}+T^{T} \Phi_{i}^{M^{a c t}}\right] \\
\beta & -\Phi_{l_{3}}^{F^{a c t} T}\left[R_{3} \Phi_{i}^{F}+R_{3} \Phi_{i}^{F^{a c t}}\right] \beta-\Phi_{l_{3}}^{F^{a c t} T}\left[S_{3} \Phi_{i}^{M}+S_{3} \Phi_{i}^{M^{a c t}}\right] \beta \\
& \left.-\Phi_{l_{2}}^{M^{a c t} T}\left[S_{2}^{T} \Phi_{i}^{F}+S_{2}^{T} \Phi_{i}^{F^{a c t}}\right] \beta-\Phi_{l_{2}}^{M^{a c t} T}\left[T_{2} \Phi_{i}^{M}+T_{2} \Phi_{i}^{M^{a c t}}\right] \beta\right\} \mathrm{d} x
\end{aligned}
$$




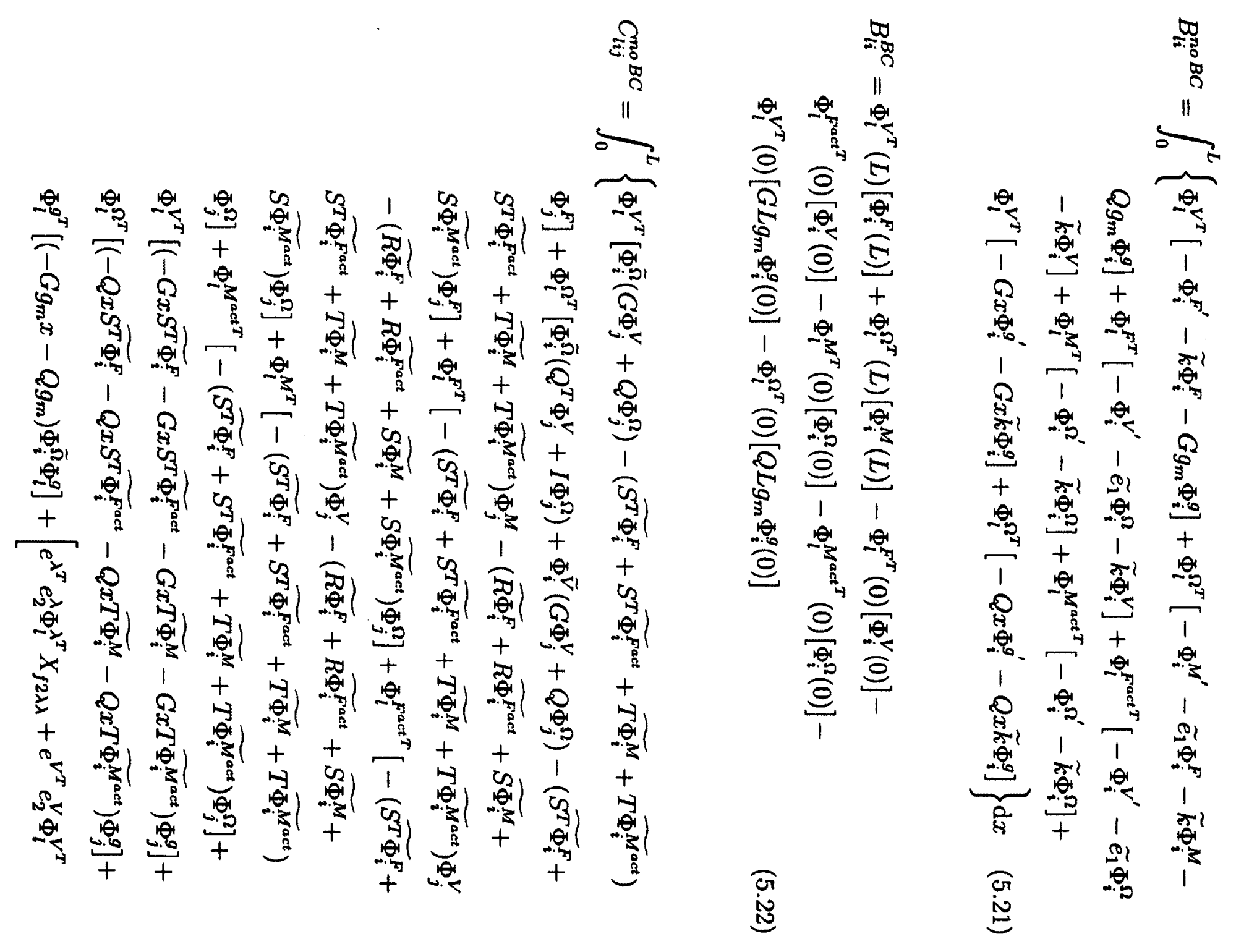




$$
\begin{aligned}
& X_{f 2 V \lambda}+e^{\Omega^{T}} e_{2}^{\Omega} \Phi_{l}^{\Omega^{T}} X_{f 2 \Omega \lambda}+e^{V^{T}} e_{3}^{V} \Phi_{l}^{V^{T}} X_{f 3 V \lambda}+e^{\lambda^{T}} e_{1}^{\lambda} \Phi_{l}^{\lambda^{T}} \\
& \left.X_{m 1 \lambda \lambda} / a b+e^{V^{T}} e_{1}^{V} \Phi_{l}^{V^{T}} X_{m 1 V \lambda} / a b+e^{\Omega^{T}} e_{1}^{\Omega} \Phi_{l}^{\Omega^{T}} X_{m 1 \Omega \lambda} / a b\right] \\
& {\left[e_{1}^{V} \Phi_{i}^{V^{T}} X_{i n f 1 V \lambda} \Phi_{j}^{\lambda}+e_{1}^{V} \Phi_{i}^{V^{T}} X_{i n f 2 V \lambda} \Phi_{j}^{\lambda}+e_{3}^{V} \Phi_{i}^{V^{T}} X_{i n f 3 V \lambda} \Phi_{j}^{\lambda}\right\} \mathrm{d} x}
\end{aligned}
$$

$$
\begin{aligned}
E_{l i / 3}^{\text {no } B C}= & \int_{0}^{L}\left\{-\Phi_{l}^{\left|V^{T} G g_{m}\right|} \Phi_{i}^{|g|}-\Phi_{l}^{\left|\Omega^{T} Q g_{m}\right|} \Phi_{i}^{|g|}\right\} \mathrm{d} x \\
F_{l}^{\text {noBC }}= & \int_{0}^{L}\left\{\Phi_{l}^{\left|V^{T} G g_{m}\right|}+\Phi_{l}^{\left|\Omega^{T} Q g_{m}\right|}\right\} \mathrm{d} x \\
F_{l}^{B C}= & \Phi_{l}^{F^{T}}(0)\left\{V^{0}\right\}+\Phi_{l}^{F^{a c t^{T}}}(0)\left\{V^{0}\right\}+\Phi_{l}^{M^{T}}(0)\left\{\Omega^{0}\right\}+ \\
& \Phi_{l}^{M^{a c t}{ }^{T}}(0)\left\{\Omega^{0}\right\}+\Phi_{l}^{V^{T}}(0) G L g_{m}\left\{g^{0}\right\}+\Phi_{l}^{\Omega^{T}}(0) Q L g_{m}\left\{g^{0}\right\}- \\
& \Phi_{l}^{V^{T}}(L)\left\{F^{L}\right\}-\Phi_{l}^{\Omega^{T}}(L)\left\{M^{L}\right\} \\
F_{l}^{C O}= & \Phi_{l}^{F_{3}}(0) \beta V_{3}^{0}+\Phi_{l}^{M_{2}}(0) \beta \Omega_{2}^{0}
\end{aligned}
$$

In the interest of brevity, the aerodynamic tensors (superscript $A E$ ) are not presented in the thesis. However, these tensors can be easily identified from Eq. 5.12 by expanding the terms containing $f^{a}$ and $m^{a}$.

Using Legendre polynomials, the internal force comparison function can be redefined as:

$$
\Phi_{l}^{F}(x)=\Psi^{F} P_{l}(x), \quad \Psi^{F}=[0,0, I, 0,0,0,0,0]_{(3 \times 24)}
$$

where $P_{l}(x)$ is the $l^{\text {th }}$ Legendre polynomial. Comparison functions for other variables, as well as comparison functions of the magnitude of variables can all be found in 
Appendix C.2.

In this study, it is assumed that the cross-sectional stiffness and inertial properties of the blade are constant along its span. It is also assumed that the aerodynamic coefficients such as $C_{l \alpha}$ do not vary with the velocity. Using these two assumptions, all constants may be taken out from the spatial integrals. Then, the only remaining terms are the following Legendre integrals that can be carried out in advance for computational efficiency:

$$
\begin{gathered}
S_{l}^{\int}=x \int_{0}^{L}\left\{P_{l}\left(\frac{x}{L}\right)\right\} \mathrm{d} x=L \int_{0}^{1}\left\{P_{l}\left(\frac{x}{L}\right)\right\} \mathrm{d} x=\left\{\begin{array}{cc}
L & \text { if } l=1 \\
0 & \text { Otherwise }
\end{array}\right\} \\
\begin{array}{c}
{ }^{d} S_{l}^{J}=\int_{0}^{L}\left\{P_{l}^{\prime}\left(\frac{x}{L}\right)\right\} \mathrm{d} x=\left\{\begin{array}{cc}
0 & \text { if } l=2 m+1 \\
2 & \text { if } l=2 m
\end{array}\right\} \\
{ }_{a} S_{l}^{J}=\int_{0}^{L}\left\{\left|P_{l}\left(\frac{x}{L}\right)\right|\right\} \mathrm{d} x=L \int_{0}^{1}\left\{\left|P_{l}\left(\frac{x}{L}\right)\right|\right\} \mathrm{d} x
\end{array} \\
D_{l i}^{\int}=\int_{0}^{L}\left\{P_{l}\left(\frac{x}{L}\right) P_{i}\left(\frac{x}{L}\right)\right\} \mathrm{d} x=L \int_{0}^{1}\left\{P_{l}\left(\frac{x}{L}\right) P_{i}\left(\frac{x}{L}\right)\right\} \mathrm{d} x \\
=\left\{\begin{array}{c}
\frac{L}{2 l-1} \quad \text { if } l=i \\
0 \quad \text { Otherwise }
\end{array}\right\} \\
D_{l i}^{\int x}=\int_{0}^{L}\left\{x P_{l}\left(\frac{x}{L}\right) P_{i}\left(\frac{x}{L}\right)\right\} \mathrm{d} x=L^{2} \int_{0}^{\frac{\pi}{2}}\left\{\sin \theta \cos \theta P_{l}(\theta) P_{i}(\theta)\right\} \mathrm{d} \theta \\
=\left\{\begin{array}{cc}
\frac{L^{2}}{4 l-2} & \text { if } l=i \\
- & \text { Otherwise }
\end{array}\right\}
\end{gathered}
$$




$$
\begin{aligned}
& { }^{d} D_{l i}^{S}=\int_{0}^{L}\left\{P_{l}\left(\frac{x}{L}\right) P_{i}^{\prime}\left(\frac{x}{L}\right)\right\} \mathrm{d} x=\int_{0}^{1}\left\{P_{l}\left(\frac{x}{L}\right) P_{i}^{\prime}\left(\frac{x}{L}\right)\right\} \mathrm{d} x \\
& =\left\{\begin{array}{cc}
2 & \text { if } i=l+1 \text { or } i=l+3 \text { or } \ldots \\
0 & \text { Otherwise }
\end{array}\right\} \\
& { }^{d} D_{l i}^{\int x}=\int_{0}^{L}\left\{x P_{l}\left(\frac{x}{L}\right) P_{i}^{\prime}\left(\frac{x}{L}\right)\right\} \mathrm{d} x=\left\{\begin{array}{cc}
L & \text { if } l<i \\
\frac{(l-1) L}{2 l-1} & \text { if } l=i \\
0 & \text { if } l>i
\end{array}\right\} \\
& { }_{a} D_{l i}^{J}=\int_{0}^{L}\left\{\left|P_{l}\left(\frac{x}{L}\right)\right|\left|P_{i}\left(\frac{x}{L}\right)\right|\right\} \mathrm{d} x=L \int_{0}^{1}\left\{\left|P_{l}\left(\frac{x}{L}\right) \| P_{i}\left(\frac{x}{L}\right)\right|\right\} \mathrm{d} x \\
& T_{l i j}^{S}=\int_{0}^{L}\left\{P_{l}\left(\frac{x}{L}\right) P_{i}\left(\frac{x}{L}\right) P_{j}\left(\frac{x}{L}\right)\right\} \mathrm{d} x=L \int_{0}^{\frac{\pi}{2}}\left\{\cos \theta P_{l}(\theta) P_{i}(\theta) P_{j}(\theta)\right\} \mathrm{d} \theta \\
& T_{l i j}^{\int x}=\int_{0}^{L}\left\{x P_{l}\left(\frac{x}{L}\right) P_{i}\left(\frac{x}{L}\right) P_{j}\left(\frac{x}{L}\right)\right\} \mathrm{d} x=L^{2} \int_{0}^{\frac{\pi}{2}}\left\{\sin \theta \cos \theta P_{l}(\theta) P_{i}(\theta) P_{j}(\theta)\right\} \mathrm{d} \theta \\
& { }^{d} T_{l i j}^{S}=\int_{0}^{L}\left\{P_{l}\left(\frac{x}{L}\right) P_{i}\left(\frac{x}{L}\right) P_{j}^{\prime}\left(\frac{x}{L}\right)\right\} \mathrm{d} x=\int_{0}^{\frac{x}{2}}\left\{L \cos \theta P_{l}(\theta) P_{i}(\theta) \frac{P_{j}^{\prime}(\theta)}{L \cos \theta}\right\} \mathrm{d} \theta \\
& =\int_{0}^{\frac{\pi}{2}}\left\{P_{l}(\theta) P_{i}(\theta) P_{j}^{\prime}(\theta)\right\} \mathrm{d} \theta \\
& { }^{d} T_{l i j}^{\int x}=\int_{0}^{L}\left\{x P_{l}\left(\frac{x}{L}\right) P_{i}\left(\frac{x}{L}\right) P_{j}^{\prime}\left(\frac{x}{L}\right)\right\} \mathrm{d} x=L \int_{0}^{\frac{\pi}{2}}\left\{\sin \theta P_{l}(\theta) P_{i}(\theta) P_{j}^{\prime}(\theta)\right\} \mathrm{d} \theta
\end{aligned}
$$

where $S_{l}^{\int}, D_{l i}^{\int}$, and $T_{l i j}^{\int}$ denote the integrals of the single, double, and triple Legendre polynomials, respectively, ${ }^{d}(\cdot)$ indicates that there is one differential Legendre polynomial in the integral, $(\cdot)^{\int x}$ indicates that there is one $x$ in the integral that is multiplied by Legendre polynomials, and $a(\cdot)$ indicated that the absolute values of Legendre polynomials appear in the integral. In addition, the following notations are 
used in the subsequent derivations:

$$
S_{l}^{0}=P_{l}(0), \quad S_{l}^{L}=P_{l}(L), \quad D_{l i}^{0}=P_{l}(0) P_{i}(0), \quad D_{l i}^{L}=P_{l}(L) P_{i}(L)
$$

It must be noted that to take out the upper limit of some of the integrals and have generic Legendre integrals applicable to all blade lengths, $L$, the following change of variable is used in some of the integrals:

$$
x=L \sin \theta \Longrightarrow \mathrm{d} x=L \cos \theta \mathrm{d} \theta
$$

Using the above Legendre integrals, tensors in Eqs. 5.19-5.27 are simplified to the following constant (in time and in space) tensors:

$$
\begin{aligned}
A_{l i}^{\text {no BC }}= & \left\{\Psi_{l}^{V^{T}}\left[G \Psi_{i}^{V}+Q \Psi_{i}^{\Omega}-J \Psi_{i}^{F}-J \Psi_{i}^{F^{a c t}}-N \Psi_{i}^{M}-N \Psi_{i}^{M^{a c t}}\right]+\right. \\
& \Psi_{l}^{\Omega^{T}}\left[Q^{T} \Psi_{i}^{V}+I \Psi_{i}^{\Omega}-B \Psi_{i}^{F}-B \Psi_{i}^{F^{a c t}}-W \Psi_{i}^{M}-W \Psi_{i}^{M^{a c t}}\right]+ \\
& \Psi_{l}^{F^{T}}\left[R \Psi_{i}^{F}+R \Psi_{i}^{F^{a c t}}+S \Psi_{i}^{M}+S \Psi_{i}^{M^{a c t}}\right]+\Psi_{l}^{F^{a c t} T}\left[R \Psi_{i}^{F}+R \Psi_{i}^{F^{a c t}}+\right. \\
& \left.S \Psi_{i}^{M}+S \Psi_{i}^{M^{a c t}}\right]+\Psi_{l}^{F^{a c t} T}\left[R^{T} \Psi_{i}^{F^{a c t}}+S \Psi_{i}^{M^{a c t}}\right]+\Psi_{l}^{M^{T}}\left[S^{T} \Psi_{i}^{F}+\right. \\
& \left.S^{T} \Psi_{i}^{F^{a c t}}+T \Psi_{i}^{M}+T \Psi_{i}^{M^{a c t}}\right]+\Psi_{l}^{M^{a c t} T}\left[S^{T} \Psi_{i}^{F}+S^{T} \Psi_{i}^{F^{a c t}}+T \Psi_{i}^{M}+\right. \\
& \left.T \Psi_{i}^{M^{a c t}}\right]+\Psi_{l}^{M^{a c t} T}\left[S^{T} \Psi_{i}^{F^{a c t}}+T^{T} \Psi_{i}^{M^{a c t}}\right]+\Psi_{l}^{g^{T}}\left[-Q g_{m} \Psi_{i}^{g}\right]+\left[e^{\lambda^{T}} e_{2}^{\lambda}\right. \\
& \Psi_{l}^{\lambda^{T}} X_{f 2 \lambda \lambda}+e^{V^{T}} e_{2}^{V} \Psi_{l}^{V^{T}} X_{f 2 V \lambda}+e^{\Omega^{T}} e_{2}^{\Omega} \Psi_{l}^{\Omega^{T}} X_{f 2 \Omega \lambda}+e^{V^{T}} e_{3}^{V} \Psi_{l}^{V^{T}} X_{f 3 V \lambda}+ \\
& \left.e^{\lambda^{T}} e_{1}^{\lambda} \Psi_{l}^{\lambda^{T}} X_{m 1 \lambda \lambda} / a b+e^{V^{T}} e_{1}^{V} \Psi_{l}^{V^{T}} X_{m 1 V \lambda} / a b+e^{\Omega^{T}} e_{1}^{\Omega} \Psi_{l}^{\Omega^{T}} X_{m 1 \Omega \lambda} / a b\right] \\
& {\left.\left[A_{i n f} \Psi_{i}^{\lambda}+C_{i n f} \Psi_{i}^{V}+D_{i n f} \Psi_{i}^{\Omega}\right]\right\} D_{l i}^{\int}+\left\{\Psi_{l}^{g^{T}}\left[-G g_{m} \Psi_{i}^{g}\right]\right\} D_{l i}^{\int x} }
\end{aligned}
$$




$$
\begin{aligned}
& A_{l i}^{C O}=\left\{-\Psi_{l}^{F^{a c t} T}\left[R^{T} \Psi_{i}^{F^{a c t}}+S \Psi_{i}^{M^{a c t}}\right] \beta-\Psi_{l}^{M^{a c t} T}\left[S^{T} \Psi_{i}^{F^{a c t}}+T^{T} \Psi_{i}^{M^{a c t}}\right] \beta\right. \\
& -\Psi_{l_{3}}^{F^{a c t} T}\left[R_{3} \Psi_{i}^{F}+R_{3} \Psi_{i}^{F^{a c t}}\right] \beta-\Psi_{l_{3}}^{F^{a c t} T}\left[S_{3} \Psi_{i}^{M}+S_{3} \Psi_{i}^{M^{a c t}}\right] \beta \\
& \left.-\Psi_{l_{2}}^{M^{a c t} T}\left[S_{2}^{T} \Psi_{i}^{F}+S_{2}^{T} \Psi_{i}^{F^{\text {act }}}\right] \beta-\Psi_{l_{2}}^{M^{a c t} T}\left[T_{2} \Psi_{i}^{M}+T_{2} \Psi_{i}^{M^{a c t}}\right] \beta\right\} D_{l i}^{S} \\
& B_{l i}^{n o B C}=\left\{\left[-\Psi^{V^{T}} \Psi^{F}-\Psi^{\Omega^{T}} \Psi^{M}-\Psi^{F^{T}} \Psi^{V}-\Psi^{F^{a c t} T} \Psi^{V}-\Psi^{M^{T}} \Psi^{\Omega}\right.\right. \\
& \left.\left.-\Psi^{M^{a c t} T} \Psi^{\Omega}\right]\right\}^{d} D_{l i}^{\int}+\left\{\left[-\Psi^{V^{T}} G \Psi^{g}-\Psi^{\Omega^{T}} Q \Psi^{g}\right]\right\}^{d} D_{l i}^{\int x}+ \\
& \left\{\left[-\Psi^{V^{T}} G \widetilde{k} \Psi^{g}-\Psi^{\Omega^{T}} Q \widetilde{k} \Psi^{g}\right]\right\} D_{l i}^{\int x}+\left\{\left[-\Psi^{V^{T}}\left(\widetilde{k} \Psi^{F}+G g_{m} \Psi^{g}\right)\right.\right. \\
& \left.-\Psi^{\Omega^{T}}\left(\widetilde{e_{1}} \Psi^{F}+\widetilde{k} \Psi^{M}+Q g_{m} \Psi^{g}\right)\right]+\left[-\Psi^{F^{T}}\left(\widetilde{e_{1}} \Psi^{\Omega}+\tilde{k} \Psi^{V}\right)\right. \\
& \left.\left.-\Psi^{F^{a c t} T}\left(\widetilde{e_{1}} \Psi^{\Omega}+\widetilde{k} \Psi^{V}\right)-\Psi^{M^{T}} \widetilde{k} \Psi^{\Omega}-\Psi^{M^{a c t} T} \widetilde{k} \Psi^{\Omega}\right]\right\} D_{l i}^{\delta} \\
& B_{l i}^{B C}=\left\{\left[\Psi^{V^{T}} \Psi^{F}+\Psi^{\Omega^{T}} \Psi^{M}\right]\right\} D_{l i}^{L}-\left\{\left[\Psi^{F^{T}} \Psi^{V}+\Psi^{F^{a c t}{ }^{T}} \Psi^{V}+\Psi^{M^{T}} \Psi^{\Omega}+\right.\right. \\
& \left.\left.\Psi^{M^{a c t} T^{T}} \Psi^{\Omega}\right]+\Psi_{l}^{V^{T}}\left[G L g_{m} \Psi_{i}^{g}\right]+\Psi_{l}^{\Omega^{T}}\left[Q L g_{m} \Psi_{i}^{g}\right]\right\} D_{l i}^{0}
\end{aligned}
$$

$$
\begin{aligned}
& C_{l i j}^{m o B C}=\left\{\Psi _ { l } ^ { V ^ { T } } \left[\Psi_{i}^{\tilde{\Omega}}\left(G \Psi_{j}^{V}+Q \Psi_{j}^{\Omega}\right)-\left(\widetilde{S^{T} \Psi_{i}^{F}}+\widetilde{S^{T} \Psi_{i}^{F^{a c t}}}+\widetilde{T \Psi_{i}^{M}}+T \widetilde{\Psi_{i}^{M^{a c t}}}\right)\right.\right. \\
& \left.\Psi_{j}^{F}\right]+\Psi_{i}^{\Omega^{T}}\left[\Psi_{i}^{\widetilde{\Omega}}\left(Q^{T} \Psi_{j}^{V}+I \Psi_{j}^{\Omega}\right)+\Psi_{i}^{\widetilde{V}}\left(G \Psi_{j}^{V}+Q \Psi_{j}^{\Omega}\right)-\left(\widetilde{S^{T} \Psi_{i}^{F}}+\right.\right. \\
& \left.\left.\widetilde{S^{T} \Psi_{i}^{\text {act }}}+\widetilde{T \Psi_{i}^{M}}+T \widetilde{\Psi_{i}^{M^{a c t}}}\right) \Psi_{j}^{M}-\left(\widetilde{R \Psi_{i}^{F}}+\widetilde{R \Psi_{i}^{F^{a c t}}}+\widetilde{S \Psi_{i}^{M}}+\widetilde{S \Psi_{i}^{M^{a c t}}}\right) \Psi_{j}^{F}\right] \\
& +\Psi_{l}^{F^{T}}\left[-\left(\widetilde{S^{T} \Psi_{i}^{F}}+\widetilde{S^{T} \Psi_{i}^{F^{a c t}}} \widetilde{T \Psi_{i}^{M}}+T \widetilde{T \Psi_{i}^{M^{a c t}}}\right) \Psi_{j}^{V}-\left(\widetilde{R \Psi_{i}^{F}}+R \widetilde{\Psi_{i}^{F^{a c t}}}+\right.\right. \\
& \left.\left.\widetilde{S \Psi_{i}^{M}}+\widetilde{S \Psi_{i}^{M^{a c t}}}\right) \Psi_{j}^{\Omega}\right]+\Psi_{l}^{F^{a c t} T}\left[-\left(\widetilde{S^{T} \Psi_{i}^{F}}+\widetilde{S^{T} \Psi_{i}^{F^{a c t}}}+\widetilde{T \Psi_{i}^{M}}+T \widetilde{\Psi_{i}^{M}}{ }^{a c t}\right)\right.
\end{aligned}
$$




$$
\begin{aligned}
& \left.\Psi_{j}^{V}-\left(\widetilde{R \Psi_{i}^{F}}+\widetilde{R \Psi_{i}^{F^{a c t}}}+\widetilde{S \Psi_{i}^{M}}+S \widetilde{S \Psi_{i}^{M^{a c t}}}\right) \Psi_{j}^{\Omega}\right]+\Psi_{l}^{M^{T}}\left[-\left(\widetilde{S^{T} \Psi_{i}^{F}}+\right.\right. \\
& \left.\left.\widetilde{S^{T} \Psi_{i}^{F^{a c t}}}+\widetilde{T \Psi_{i}^{M}}+T \widetilde{\Psi_{i}^{M^{a c t}}}\right) \Psi_{j}^{\Omega}\right]+\Psi_{l}^{M^{a c t} T}\left[-\left(\widetilde{S^{T} \Psi_{i}^{F}}+\widetilde{S^{T} \Psi_{i}^{F^{a c t}}}+\right.\right. \\
& \left.\left.\widetilde{T \Psi_{i}^{M}}+T \widetilde{\Psi_{i}^{M^{a c t}}}\right) \Psi_{j}^{\Omega}\right]+\Psi_{l}^{g^{T}}\left[-G g_{m} \Psi_{i}^{\widetilde{\Omega}_{l}} \Psi_{l}^{g}\right]+\left[e^{\lambda^{T}} e_{2}^{\lambda} \Psi_{l}^{\lambda^{T}} X_{f 2 \lambda \lambda}+\right. \\
& e^{V^{T}} e_{2}^{V} \Psi_{l}^{V^{T}} X_{f 2 V \lambda}+e^{\Omega^{T}} e_{2}^{\Omega} \Psi_{l}^{\Omega^{T}} X_{f 2 \Omega \lambda}+e^{V^{T}} e_{3}^{V} \Psi_{l}^{V^{T}} X_{f 3 V \lambda}+e^{\lambda^{T}} e_{1}^{\lambda} \Psi_{l}^{\lambda^{T}} \\
& \left.X_{m 1 \lambda \lambda} / a b+e^{V^{T}} e_{1}^{V} \Psi_{l}^{V^{T}} X_{m 1 V \lambda} / a b+e^{\Omega^{T}} e_{1}^{\Omega} \Psi_{l}^{\Omega^{T}} X_{m 1 \Omega \lambda} / a b\right]\left[e_{1}^{V} \Psi_{i}^{V^{T}}\right. \\
& \left.\left.X_{i n f 1 V \lambda} \Psi_{j}^{\lambda}+e_{1}^{V} \Psi_{i}^{V^{T}} X_{i n f 2 V \lambda} \Psi_{j}^{\lambda}+\epsilon_{3}^{V} \Psi_{i}^{V^{T}} X_{i n f 3 V \lambda} \Psi_{j}^{\lambda}\right]\right\} T_{l i j}^{\int}+\left\{\Psi_{l}^{V^{T}}[(\right. \\
& \left.\left.-G \widetilde{S^{T} \Psi_{i}^{F}}-G S^{T \Psi_{i}^{F^{a c t}}}-G \widetilde{T \Psi_{i}^{M}}-G T \widetilde{\Psi_{i}^{M^{a c t}}}\right) \Psi_{j}^{g}\right]+\Psi_{l}^{\Omega^{T}}\left[\left(-Q \widetilde{S^{T} \Psi_{i}^{F}}\right.\right. \\
& \left.\left.\left.-Q S^{T} \widetilde{\Psi_{i}^{F^{a c t}}}-Q \widetilde{T \Psi_{i}^{M}}-Q T \widetilde{\Psi_{i}^{M^{a c t}}}\right) \Psi_{j}^{g}\right]+\Psi_{l}^{g^{T}}\left[-Q g_{m} \Psi_{i}^{\tilde{\Omega}} \Psi_{l}^{g}\right]\right\} T_{l i j}^{\int x}
\end{aligned}
$$

$E_{l i / 3}^{n o B C}=\left\{-\Psi_{l}^{\left|V^{T} G g_{m}\right|} \Psi_{i}^{|g|}-\Psi_{l}^{\left|\Omega^{T} Q g_{m}\right|} \Psi_{i}^{|g|}\right\}_{a} D_{l i}^{\int}$

$$
F_{l}^{n o B C}=\left\{\Psi_{l}^{\left|V^{T} G g_{m}\right|}+\Psi_{l}^{\left|\Omega^{T} Q g_{m}\right|}\right\}_{a} S_{l i}^{f}
$$

$$
\begin{aligned}
F_{l}^{B C}= & \left\{\Psi_{l}^{F^{T}}\left\{V^{0}\right\}+\Psi_{l}^{F^{a c t}}\left\{V^{0}\right\}+\Psi_{l}^{M^{T}}\left\{\Omega^{0}\right\}+\Psi_{l}^{M^{a c t}} \cdot\left\{\Omega^{0}\right\}+\right. \\
& \left.\Psi_{l}^{V^{T}} G L g_{m}\left\{g^{0}\right\}+\Psi_{l}^{\Omega^{T}} Q L g_{m}\left\{g^{0}\right\}\right\} S_{l}^{0}- \\
& \left\{\Psi_{l}^{V^{T}}\left\{F^{L}\right\}+\Psi_{l}^{\Omega^{T}}\left\{M^{L}\right\}\right\} S_{l}^{L}
\end{aligned}
$$

$$
F_{l}^{C O}=\left\{\Psi_{l}^{F_{3}} \beta V_{3}^{0}+\Psi_{l}^{M_{2}} \beta \Omega_{2}^{0}\right\} S_{l}^{0}
$$

Eqs. 5.13-5.18 and 5.43-5.51 provide all the necessary information to define the BSP formulation in the standard state-space form $\dot{x}=f(x, t)$. In the next section, the standard output equation $y=g(x, t)$ will be obtained by discretization of the 
output equations using the Galerkin method.

\subsection{Output Equations}

In the BSP problem, the output of interest is the blade tip displacement. This output is to be expressed in terms of state variables. The necessary relations to obtain this displacement and the associated rotation matrices were presented in Section 4.4 and are repeated here for convenience:

$$
\begin{aligned}
& C^{b a^{\prime}}=-\widetilde{k} C^{b a} \\
& C^{B a^{\prime}}=-(\widetilde{k+\kappa}) C^{B a} \\
& C^{B b}=C^{B a} \times C^{b a^{T}} \\
& \gamma=C^{B b}\left(e_{1}+U^{\prime}+\widetilde{k} U\right)-e_{1}
\end{aligned}
$$

where $C^{b a}$ is the rotation matrix from the untwisted, uncurved, undeformed reference frame to the twisted, curved, undeformed frame; $C^{B a}$ is the rotation matrix from the untwisted, uncurved, undeformed frame to the twisted, curved, deformed frame, and $U$ is the displacement of the twisted, curved, deformed frame with respect to the twisted, curved, undeformed frame. These equations are solvable analytically. It is shown in calculus books such as Ref. [39], that a linear first order ordinary differential equation of the form of $y^{\prime}(x)+p(x) y(x)=z(x)$ can be solved as:

$$
y(x)=e^{-\int p(x) \mathrm{d} x}\left[c+\int z(x) e^{\int p(x) \mathrm{d} x} \mathrm{~d} x\right]
$$

where $e^{()}$indicates the exponential function, and $c$ is a constant obtainable from the initial value of the function $y(x)$. Using this general solution, Eqs. 5.52 and 5.53 can 
be solved as follows:

$$
\begin{aligned}
C^{b a^{\prime}} & =-\widetilde{k} C^{b a} \Longrightarrow C^{b a}=e^{-\tilde{k} x} \\
C^{B a^{\prime}} & =-(\widetilde{k+\kappa}) C^{B a} \Longrightarrow C^{B a}=e^{-\tilde{k} x-\int \tilde{k} \mathrm{~d} x} \\
& =e^{-\tilde{k} x-\int\left(\widetilde{S^{T} F}+S^{\widetilde{T} F^{a}}+\widetilde{T M}+T \widetilde{M^{a} c t}\right) \mathrm{d} x}
\end{aligned}
$$

Substituting these solutions in Eq. 5.55 and expressing $\gamma$ in terms of the primary variables one can get:

$$
\begin{aligned}
R F+R F^{a c t}+S M+S M^{a c t}+e_{1}= & \left(e^{-\left(\widetilde{k}+\widetilde{k}^{T}\right) x-\int\left(\widetilde{S^{T F}}+S^{T F^{a c t}}+\widetilde{T M}+T \widetilde{M^{a c t}}\right) \mathrm{d} x}\right) \\
& \left(e_{1}+U^{\prime}+\widetilde{k} U\right)
\end{aligned}
$$

However, $\tilde{k}+\tilde{k}^{T}=0$ for all arbitrary vectors $k$. Therefore, Eq. 5.59 can be simplified to:

$$
\begin{aligned}
R F+R F^{a c t}+S M+S M^{a c t}+e_{1}= & \left(e^{-\int \widetilde{S^{T} F}+S^{T F^{a c t}}+\widetilde{T M}+T \widetilde{M^{a c t}} \mathrm{~d} x}\right) \\
& \left(e_{1}+U^{\prime}+\widetilde{k} U\right)
\end{aligned}
$$

In reference to Eq. 5.56, defining:

$$
\begin{aligned}
& p(x)=\tilde{k} \\
& z(x)=\frac{R F+R F^{a c t}+S M+S M^{a c t}+e_{1}-e^{-\int \widetilde{S^{T F}}+S^{T F^{a c t}}+\widetilde{T M}+\widetilde{M^{a c t}} d x} e_{1}}{e^{-\int\left(\widetilde{S^{T} F}+S^{\widetilde{T} F^{a c t}}+\widetilde{T M}+T \widetilde{M^{a c t}} \mathrm{~d} x\right.}}
\end{aligned}
$$


the solution for displacement $(U)$ is obtained as:

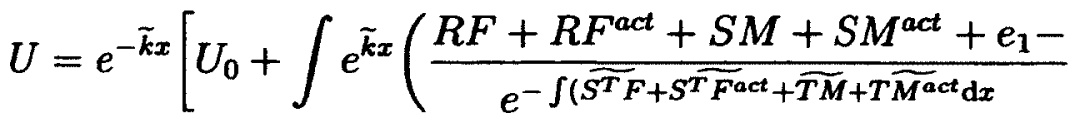

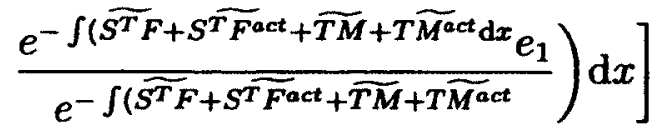

where $U_{0}$ can be obtained using zero blade root structural displacement as follows

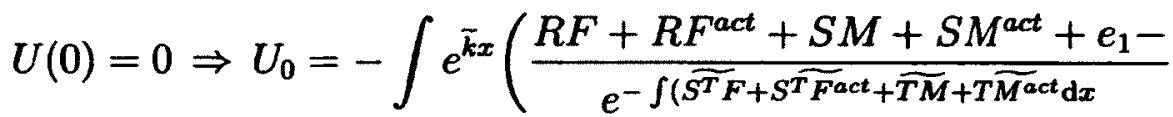

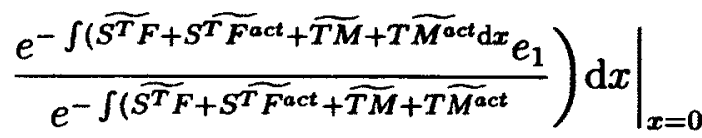

Although the above equations give the exact analytical solution for the displacement vector along the blade, these equations are not realizable since the indefinite integration of the exp function in most cases does not exist. Another disadvantage of this solution is its demand for high computational effort as it contains two integrations and the inverse of a $3 \times 3$ matrix with exponential terms. Due to these deficiencies associated with the exact analytical solution, similar to the state equations, Galerkin discretization is used to obtain the output displacement and rotation in terms of the state variables.

As is shown in Ref. [28], Eqs. 5.52-5.54 can be combined to result in the following strain-displacement relation:

$$
\begin{aligned}
& \gamma=C^{B b}\left(e_{1}+U^{\prime}+\widetilde{k} U\right)-e_{1} \\
& \widetilde{\kappa}=-C^{B b^{\prime}} C^{B b^{T}}+C^{B b} \widetilde{k} C^{B b^{T}}-\widetilde{k}
\end{aligned}
$$

Taking advantage of orthogonality of the direction cosine matrix $C^{B b}\left(C^{B b} C^{B b^{T}}=\right.$ $C^{B b^{T}} C^{B b}=I$ ), and premultiplying Eq. 5.63 by $C^{B b^{T}}$ and postmultiplying Eq. 5.64 
by $C^{B b}$ one obtains:

$$
\begin{aligned}
& 0=U^{\prime}+\widetilde{k} U+e_{1}-C^{B b^{T}}\left(e_{1}+\gamma\right) \\
& 0=C^{B b^{\prime}}+(\widetilde{k+\kappa}) C^{B b}-C^{B b} \widetilde{k}
\end{aligned}
$$

These vector equations, in fact, result in 12 scalar equations for 12 unknowns (3 displacements and 9 rotation matrix elements). To solve these equations using the Galerkin method, similar to the state variables, the output variables $U$ and $C^{B b}$ are expressed in terms of products of their comparison and temporal functions, i.e.,

$$
\begin{aligned}
U & =\sum_{i=1}^{n c} \Phi_{i}^{U}(x) U_{i}(t) \\
C^{B b} & =\sum_{i=1}^{n c} \Phi_{i}^{C^{B b}}(x) C_{i}^{B b}(t)
\end{aligned}
$$

Substituting the above equations in Eqs. 5.65 and 5.66, and expanding the state variables in terms of their comparison functions, one obtains the following equations:

$$
\begin{aligned}
0= & \sum_{i=1}^{n c}\left(\Phi_{i}^{U^{\prime}}+\widetilde{k} \Phi_{i}^{U}\right) U_{i}(t)+e_{1}-\sum_{i=1}^{n c} \Phi_{i}^{C^{B b}} C_{i}^{B b^{T}}(t)\left[e_{1}+\right. \\
& \left.\sum_{j=1}^{n c}\left(R \Phi_{j}^{F}+R \Phi_{j}^{F^{a c t}}+S \Phi_{j}^{M}+S \Phi_{j}^{M^{a c t}}\right) q_{j}(t)\right] \\
0= & \sum_{i=1}^{n c}\left(\Phi_{i}^{C^{B b}}+\widetilde{k} \Phi_{i}^{C^{B b}}\right) C_{i}^{B b}(t)-\sum_{i=1}^{n c} \Phi_{i}^{C^{B b}} C_{i}^{B b}(t) \widetilde{k}+ \\
& \sum_{j=1}^{n c}\left(\widetilde{S^{T} \Phi_{j}^{F}}+\widetilde{S^{T} \Phi_{j}^{F^{a c t}}}+\widetilde{T \Phi_{j}^{M}}+T \widetilde{\Phi_{j}^{M^{a c t}}}\right) q_{j}(t) \sum_{i=1}^{n c} \Phi_{i}^{C^{B b}} C_{i}^{B b}(t)
\end{aligned}
$$

To remove the comparison functions, the above equations can be integrated along the blade span. This integration gives displacement and rotation temporal functions, $U_{i}(t)$ and $C_{i}^{B b}(t)$, in terms of the generalized temporal function $q_{i}(t)$. Integrating along the span, and expanding the summations, the following temporal functions are 
obtained:

$$
\begin{aligned}
0=\int_{0}^{L} & \left\{\left(\Phi_{i}^{U^{\prime}}+\widetilde{k} \Phi_{i}^{U}\right) U_{i}+e_{1} \delta_{i-1}-C_{i}^{B b} \Phi_{i}^{C^{B b} T} e_{1}-\right. \\
& \left.C_{i}^{B b} \Phi_{i}^{C^{B b} T}\left(R \Phi_{j}^{F}+R \Phi_{j}^{F^{a c t}}+S \Phi_{j}^{M}+S \Phi_{j}^{M^{a c t}}\right) q_{j}\right\} \mathrm{d} x \\
0=\int_{0}^{L}\{ & \left(\Phi_{i}^{C^{B b}}+\widetilde{k} \Phi_{i}^{C^{B b}}\right) C_{i}^{B b}-\Phi_{i}^{C^{B b}} C_{i}^{B b} \widetilde{k}+ \\
& \left.\left(\widetilde{S^{T} \Phi_{j}^{F}}+\widetilde{S^{T} \Phi_{j}^{F^{a c t}}}+\widetilde{T \Phi_{j}^{M}}+T \widetilde{\Phi_{j}^{M^{a c t}}}\right) q_{j} \Phi_{i}^{C^{B b}} C_{i}^{B b}\right\} \mathrm{d} x
\end{aligned}
$$

where the argument $(t)$ of the generalized temporal function $q_{i}(t)$ is dropped for simplicity, and $\delta_{i-1}$ denotes the Kronecker delta, equal to 1 if $i=1$ and 0 otherwise.

It can be shown that in the above equations the following equality is valid:

$$
\Phi_{i}^{C^{B b}} C_{i}^{B b}=C_{i}^{B b} \Phi_{i}^{C^{B b}}
$$

Therefore, Eqs. 5.71 and 5.72 can be rewritten as:

$$
\begin{aligned}
& 0= \int_{0}^{L}\left\{\left(\Phi_{i}^{U^{\prime}}+\tilde{k} \Phi_{i}^{U}\right) U_{i}+e_{1} \delta_{i-1}-C_{i}^{B b} \Phi_{i}^{C^{B b}} e_{1}-\right. \\
&\left.C_{i}^{B b} \Phi_{i}^{C^{B b}}\left(R \Phi_{j}^{F}+R \Phi_{j}^{F^{a c t}}+S \Phi_{j}^{M}+S \Phi_{j}^{M^{a c t}}\right) q_{j}\right\} \mathrm{d} x \\
& 0= \int_{0}^{L}\left\{\left(\Phi_{i}^{C^{B b}}+\tilde{k} \Phi_{i}^{C^{B b}}\right) C_{i}^{B b}-C_{i}^{B b} \Phi_{i}^{C^{B b}} \widetilde{k}+\right. \\
&\left.\left(\widetilde{S^{T} \Phi_{j}^{F}}+\widetilde{S^{T} \Phi_{j}^{F^{a c t}}}+\widetilde{T \Phi_{j}^{M}}+T \widetilde{\Phi_{j}^{M^{a c t}}}\right) \Phi_{i}^{C^{B b}} q_{j} C_{i}^{B b}\right\} \mathrm{d} x
\end{aligned}
$$

Using Legendre polynomials for the displacement and the rotation comparison functions and assuming constant structural and inertial properties for the blade along 
its span, Eqs. 5.74 and 5.75 can be summarized as the following two tensor equations:

$$
\begin{aligned}
& 0=H_{i i} U_{i}+\Delta_{i}-C_{i}^{B b} K_{i}-C_{i}^{B b} N_{i j} q_{j} \\
& 0=X_{i i} C_{i}^{B b}-C_{i}^{B b} Y_{i i}+Z_{j i} q_{j} C_{i}^{B b}
\end{aligned}
$$

where

$$
\begin{aligned}
& H_{i i}=\int_{0}^{L}\left\{\Phi_{i}^{U^{\prime}}+\widetilde{k} \Phi_{i}^{U}\right\} \mathrm{d} x=\left[\Psi^{U}\right]^{d} S_{i}^{J}+\left[\widetilde{k} \Psi^{U}\right] S_{i}^{J} \\
& \Delta_{i}=\int_{0}^{L}\left\{e_{1} \delta_{i-1}\right\} \mathrm{d} x=\left\{\begin{array}{cc}
L e_{1} & \text { if } i=1 \\
0 & \text { Otherwise }
\end{array}\right\} \\
& K_{i}=\int_{0}^{L}\left\{\Phi_{i}^{C^{B b}} e_{1}\right\} \mathrm{d} x=\left[\Psi^{C^{B b}} e_{1}\right] S_{i}^{J} \\
& N_{i j}=\int_{0}^{L}\left\{\Phi_{i}^{C^{B b}}\left(R \Phi_{j}^{F}+R \Phi_{j}^{F^{a c t}}+S \Phi_{j}^{M}+S \Phi_{j}^{M^{a c t}}\right)\right\} \mathrm{d} x= \\
& {\left[\Psi^{C^{B b}}\left(R \Psi^{F}+R \Psi^{F^{a c t}}+S \Psi^{M}+S \Psi^{M^{a c t}}\right)\right] D_{i j}^{f}} \\
& X_{i i}=\int_{0}^{L}\left\{\Phi_{i}^{C^{B b^{\prime}}}+\widetilde{k} \Phi_{i}^{C^{B b}}\right\} \mathrm{d} x=\left[\Psi^{C^{B b}}\right]^{d} S_{i}^{S}+\left[\widetilde{k} \Psi^{C^{B b}}\right] S_{i}^{S} \\
& Y_{i i}=\int_{0}^{L}\left\{\Phi_{i}^{C^{B b}} \widetilde{k}\right\} \mathrm{d} x=\left[\Psi^{C^{B b}} \tilde{k}\right] S_{i}^{S} \\
& \left.Z_{j i}=\int_{0}^{L}\left\{\widetilde{\left(S^{T} \Phi_{j}^{F}\right.}+\widetilde{S^{T} \Phi_{j}^{F^{a c t}}}+\widetilde{T \Phi_{j}^{M}}+\widetilde{T \Phi_{j}^{M^{a c t}}}\right) \Phi_{i}^{C^{B b}}\right\} \mathrm{d} x= \\
& {\left[\widetilde{S^{T} \Psi^{F}}+S^{T} \widetilde{\Psi^{F}}+\widetilde{T \Psi^{M}}+T \widetilde{\Psi^{M^{a c t}}}\right] \Psi^{C^{B b}} D_{j i}^{\int}}
\end{aligned}
$$

Eqs. 5.13-5.84 provide all the necessary equations to obtain the displacements and the rotation matrix output variables as functions of the state variables. The resulting 
closed-loop equations can be cast in the standard form of nonlinear systems as

$$
\begin{aligned}
& \dot{x}=f(x, t) \\
& y=g(x, t)
\end{aligned}
$$

and can be solved using nonlinear ODE solvers for selected boundary values (Engage/disengage profiles). The solution of these equations and the results are discussed in the next chapter. 


\section{Chapter 6}

\section{Results and Discussion}

In this chapter, the performance of the proposed feedback control strategy on the BSP is evaluated in three steps. In the first step, to validate the discretized model, the structural frequencies of a prismatic cantilevered beam obtained by the Galerkin Discretization Method is compared to those obtained by the finite element and the exact methods. The accuracy of the Galerkin method is further tested by observing the fulfilment of the boundary values and constraint equations. In the next step, the proposed feedback controller is applied to the model and the results are compared to those obtained with no actuation in the blade in two cases: constant and variable rotating velocity. In the final step, the performance of the feedback controller is tested on a comprehensive BSP model that has been validated against experimental data.

\subsection{Galerkin Method Validation}

As a preliminary validation of the Galekin discretization method, a prismatic cantilevered beam with zero extensional and shear flexibilities was analyzed in Ref. [25] and the results are reproduced in this work. The structural and inertial properties of the beam are shown in Table 6.1. The structural frequencies of the beam are shown in Table 6.2. To obtain the structural frequency, all external loads as well as inflow 
and control effects are dropped in Eq. 5.13 and the resultant equation is linearized about the steady state solution. For linearization, Taylor series expansion, in which the high order nonlinear terms are ignored, is employed.

In Table 6.2, frequencies predicted by the Galerkin method with 10 comparison functions (120 states) [25] are compared with those predicted by a FEM with 10 nodes and first order shape functions (120 states) [40] and those obtained by the exact solution (FEM with 2 nodes and 15th-order shape function) [40], for two rotating and non-rotating beam cases. The reason to use similar number of comparison functions and FEM nodes was to have comparable complexities in equations (identical number of states). As can be seen, while the FEM predication has up to $10 \%$ error in higher bending modes, the current Galerkin method predictions are identical to the exact solution to three significant digits. It should be noted that the simulation results obtained with less than 10 comparison functions have shown that as the number of used comparison functions increases, the results converge to the exact solution. This example proves the ability of the Galerkin method to predict the structural deflections of rotating beams.

To further evaluate the developed model and specifically the Galerkin discretization method, satisfaction of the boundary value and magnitude constraints in the equations are checked at constant and variable angular velocities. For this purpose, the Active Twist Rotor (ATR) blade is employed. The ATR blade resulted from a joint program between NASA Langley, the US Army Research Office and, the University of Michigan/MIT. It was mainly designed to investigate the vibration damping capability of the embedded active piezo fibres [40]. The span length of the ATR was $1.397 \mathrm{~m}$ and a NACA 0012 airfoil with a chord length of $0.137 \mathrm{~m}$ was used as its cross section. The aerodynamic properties of the cross section are shown in Table 6.3 and 
Table 6.1: Prismatic cantilevered beam properties [25].

\begin{tabular}{|lc|}
\hline \hline Parameter & Value \\
\hline Span length & $16 \mathrm{~m}$ \\
Chord length & $1 \mathrm{~m}$ \\
Mass per unit length & $0.75 \mathrm{~kg} / \mathrm{m}$ \\
Moment of inertia per unit length & $0.1 \mathrm{~kg} \cdot \mathrm{m}$ \\
Spanwise elastic axis location (center of rotation) & $50 \%$ chord \\
Center of mass location & $50 \%$ chord \\
Flapwise bending stiffness & $2 \times 10^{4} \mathrm{~N} \cdot \mathrm{m}^{2}$ \\
Torsional stiffness & $1 \times 10^{4} \mathrm{~N} \cdot \mathrm{m}^{2}$ \\
Chordwise bending stiffness & $4 \times 10^{6} \mathrm{~N} \cdot \mathrm{m}^{2}$ \\
Extensional stiffness & $\infty$ \\
Shear stiffness & $\infty$ \\
\hline
\end{tabular}

Table 6.2: Prismatic cantilevered beam structural frequencies [25].

\begin{tabular}{|c|ccc|}
\hline \hline Mode (rad/s) & $\begin{array}{c}\text { Exact } \\
\text { (FEM, 15th-order shape function) })\end{array}$ & $\begin{array}{c}\text { Spectral Galerkin } \\
\text { (10 comparison functions) }\end{array}$ & $\begin{array}{c}\text { FEM } \\
(10 \text { nodes })\end{array}$ \\
\hline \multicolumn{4}{|c|}{ Angular Velocity = 0 rad/s } \\
\hline $1^{\text {st } \text { bending }}$ & 2.243 & 2.243 & 2.252 \\
$2^{\text {nd }}$ bending & 14.06 & 14.06 & 14.74 \\
$3^{\text {rd }}$ bending & 39.36 & 39.36 & 44.94 \\
$1^{\text {st }}$ torsion & 31.05 & 31.05 & 31.12 \\
$2^{\text {nd } \text { torsion }}$ & 93.14 & 93.14 & 95.32 \\
\hline \multicolumn{5}{|c|}{ Angular Velocity $=3.189 \mathrm{rad} / \mathrm{s}$} \\
\hline $1^{\text {st }}$ bending & 4.114 & 4.114 & 4.110 \\
$2^{\text {nd }}$ bending & 16.23 & 16.23 & 16.88 \\
$3^{\text {rd }}$ bending & 41.59 & 41.59 & 47.12 \\
\hline
\end{tabular}


the structural and inertial properties of the ATR in basic SI units are as follows:

$$
R=10^{-7}\left[\begin{array}{ccc}
6.4375 & 0.0000 & 0.0000 \\
0.0000 & 49.2620 & 0.0000 \\
0.0000 & 0.0000 & 443.8900
\end{array}\right], S=10^{-6}\left[\begin{array}{ccc}
0.000 & 0.000 & 5.542 \\
0.000 & 0.000 & 0.000 \\
186.210 & 0.000 & 0.000
\end{array}\right]
$$

$T=10^{-4}\left[\begin{array}{ccc}290.86 & 0.00 & 0.00 \\ 0.00 & 250.38 & 0.00 \\ 0.00 & 0.00 & 926.40\end{array}\right], G=10^{-1}\left[\begin{array}{ccc}6.931 & 0.000 & 0.000 \\ 0.000 & 6.931 & 0.000 \\ 0.000 & 0.000 & 6.931\end{array}\right]$

$$
Q=10^{-4}\left[\begin{array}{ccc}
0.0000 & 0.0000 & 4.7999 \\
0.0000 & 0.0000 & 0.0000 \\
-4.7999 & 0.0000 & 0.0000
\end{array}\right], I=10^{-6}\left[\begin{array}{ccc}
376.65 & 0.000 & 0.000 \\
0.000 & 6.463 & 0.000 \\
0.000 & 0.000 & 370.180
\end{array}\right]
$$

To validate the Galerkin method, a rotating ATR blade with angular velocity $\Omega^{0}=\{0 ; 0 ; 10\}$ is assumed. The cther boundary values are as follows:

$$
V^{0}=\left[\begin{array}{l}
0 \\
0 \\
0
\end{array}\right], \quad F^{L}=\left[\begin{array}{l}
0 \\
0 \\
0
\end{array}\right], \quad M^{L}=\left[\begin{array}{l}
0 \\
0 \\
0
\end{array}\right], \quad g^{0}=\left[\begin{array}{c}
0 \\
0 \\
-1
\end{array}\right]
$$


Table 6.3: Aerodynamic properties of the ATR airfoil.

\begin{tabular}{|lc|}
\hline \hline Parameter & Value \\
\hline$c l_{\alpha}$ & 5.618 \\
$c l_{0}$ & 0 \\
$c d_{0}$ & 0.01 \\
$c m_{0}$ & 0 \\
$c m_{\alpha}$ & -0.28 \\
$\rho$ & $1.1455 \mathrm{~kg} / \mathrm{m}^{3}$ \\
$b$ & $0.06843 \mathrm{~m}$ \\
$a$ & -0.5 \\
\hline
\end{tabular}

Eq. 5.13 was formed with 8 comparison functions and the resultant 192 nonlinear ODEs with zero initial values were implemented and solved in the MATLAB/SIMULINK environment with the ODE15S solver, which was developed for stiff systems with a high number of states.

Figs. 6.1-6.5 show time histories of the state variables $V, \Omega$, and $g$ at the blade root, and $F$ and $M$ at the blade tip. As is shown, all boundary values are satisfied within the first second. Similar simulations were performed for different boundary values and the results had similar levels of acceptability. These results also indicated that 6 to 8 comparison functions are required to satisfy the boundary values. In addition to successful prediction of the structural frequencies, satisfaction of the boundary values of the equations is another measure of the validity of the formulation. 


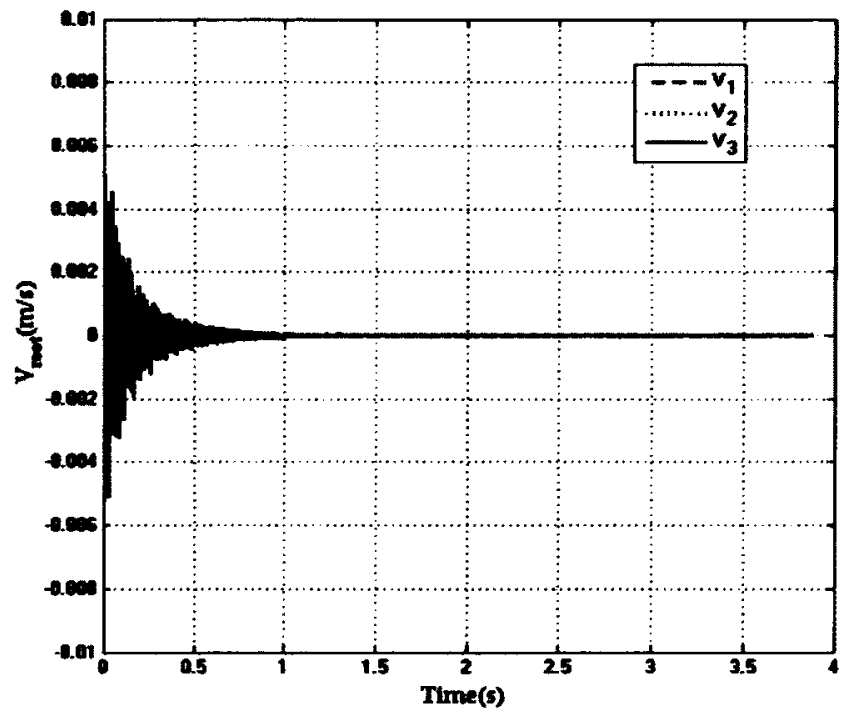

Figure 6.1: Linear velocity at the blade root.

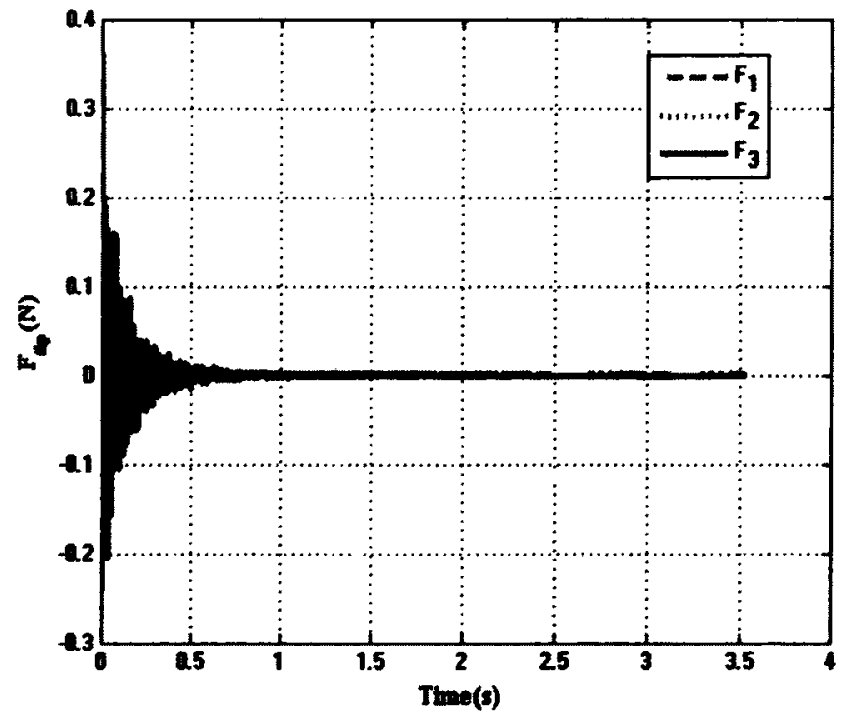

Figure 6.3: Internal force at the blade tip

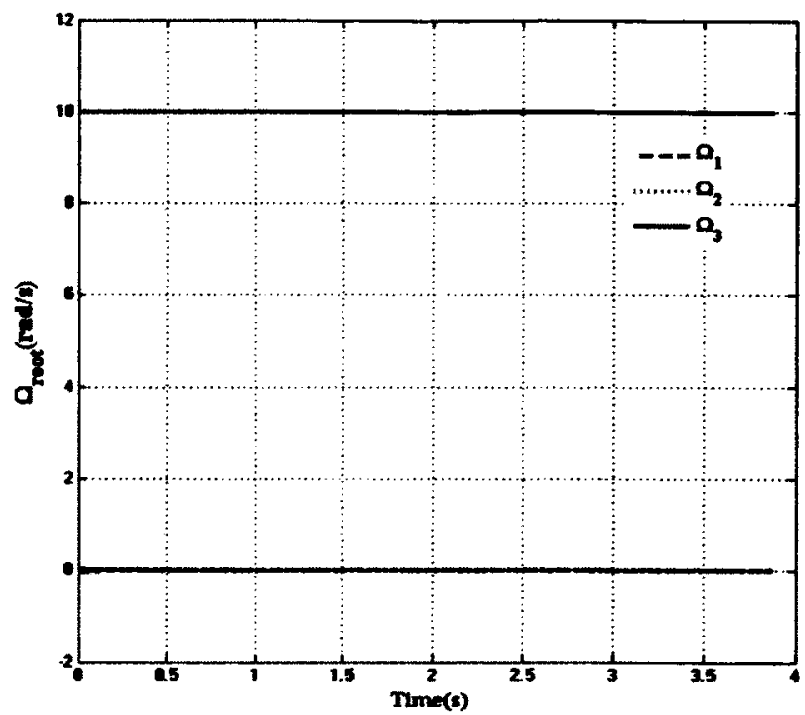

Figure 6.2: Angular velocity at the blade root.

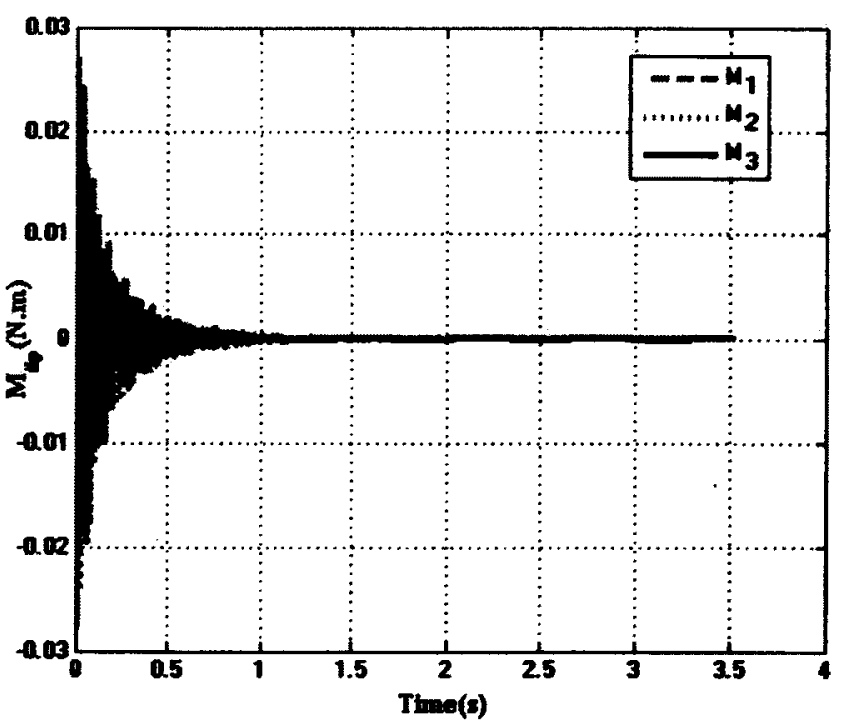

Figure 6.4: Internal moment at blade tip. 


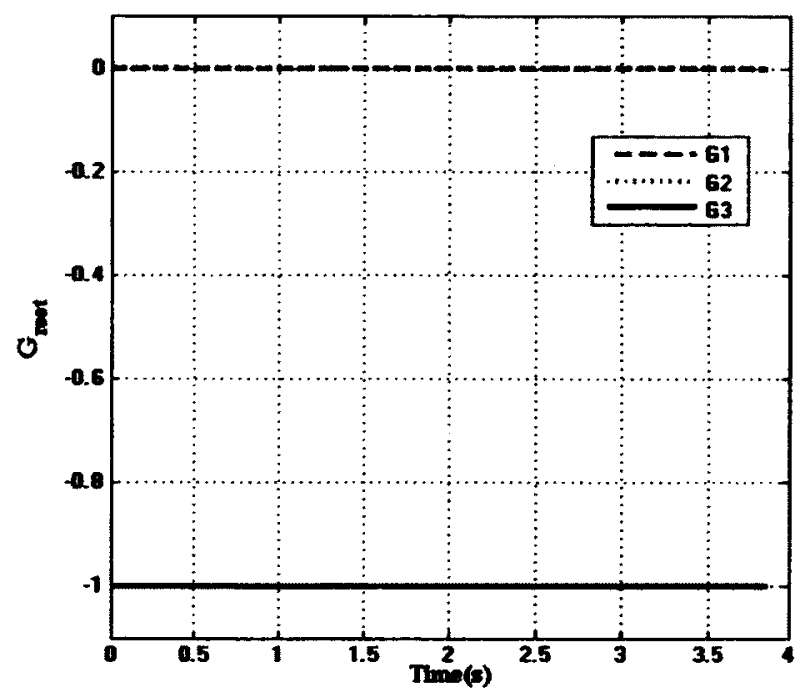

Figure 6.5: Gravity unit vector at the blade root.

\subsection{Controller Effectiveness in the Reduced BSP Model}

The BSP feedback control proposed in this research is designed based on a reduced BSP model, in which the effects of the articulation mechanism, non-uniform winds, ship motions, and droop/flap stops are neglected. In this section, the performance of the proposed Lyapunov controller is tested on this reduced BSP model.

For simulation purposes, the ATR blade introduced in the previous section with similar boundary values was employed. In Ref. [40], it is mentioned that the actuation voltage of the ATR is limited to $\pm 1500 \mathrm{~V}$. However, given the advances in piezoelectric technology over the past 12 years since the ATR blade was developed, for the purpose of this study it was decided to set the actuation limit to $\pm 2500 \mathrm{~V}$. This actuation voltage has been providable on an experimental active rotor blade [41]. The initial twist of the blade is zero and 8 comparison functions are used for discretization. The 
piezoelectric coefficient vectors $Y^{F}$ and $Y^{M}$ in basic SI units are as follows:

$$
Y^{F}=10^{-2}\left[\begin{array}{c}
5.67 \\
0.00 \\
-0.23
\end{array}\right], \quad Y^{M}=10^{-4}\left[\begin{array}{c}
5.42 \\
3.20 \\
27.70
\end{array}\right]
$$

Figs. 6.6 and 6.7 compare time histories of the blade tip displacements with and without the controller, where the controller design factor $\beta=1.5$ was used. The significant reduction of the blade tip displacement, which can be recognized by comparing these two figures, proves the potential of the proposed control strategy for countering the BSP structural displacements.

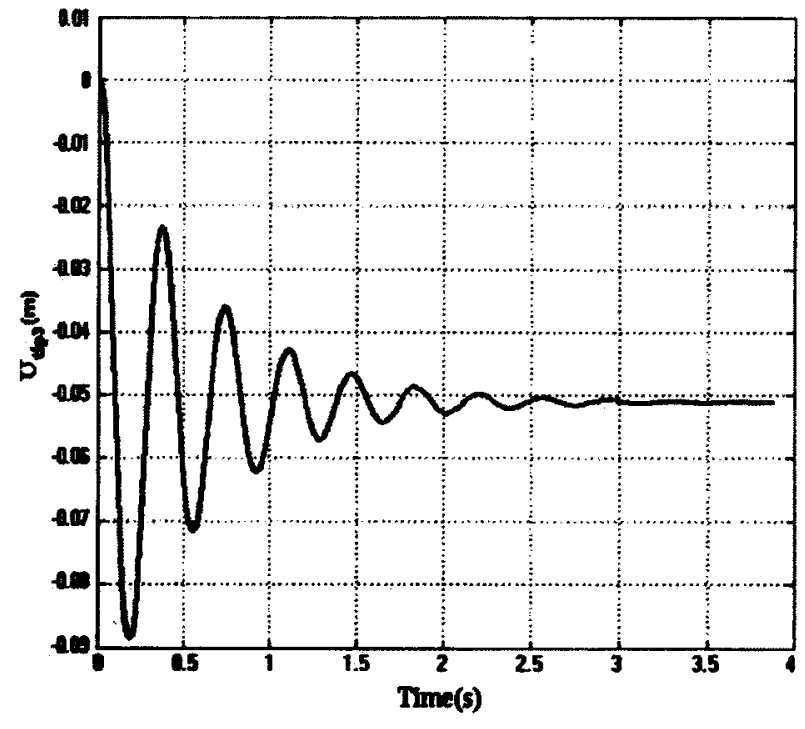

Figure 6.6: Uncontrolled elastic tip displacements of the blade.

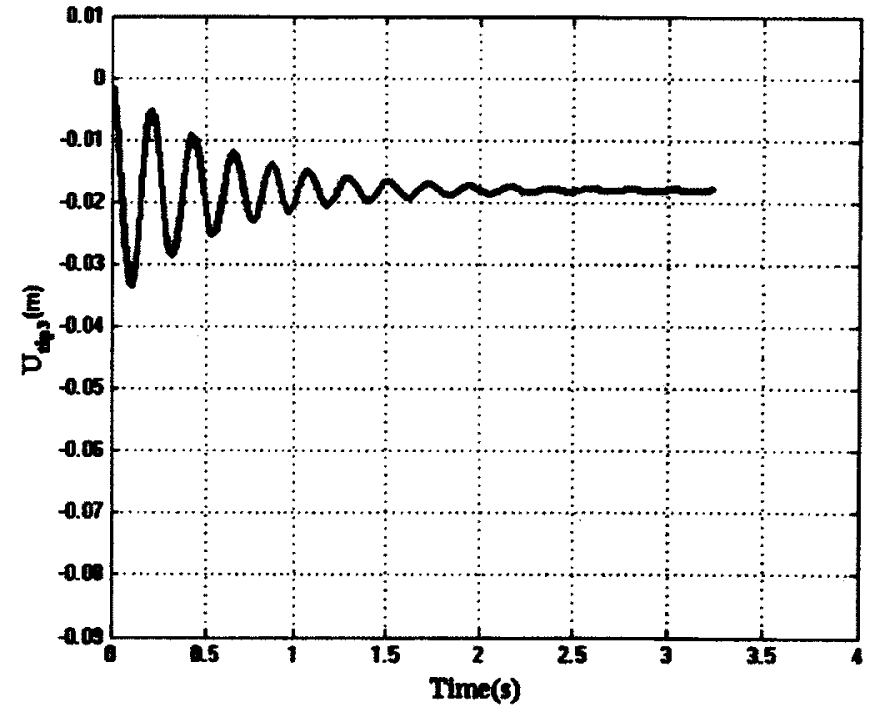

Figure 6.7: Controlled elastic tip displacements of the blade.

To assess the performance of the controller in engagement/disengagement operations, a variable velocity boundary condition based on Eq. 3.48 with disengagement time $t_{d i s}=10 / 3 \mathrm{~s}$ is considered. The initiation of the disengagement operation is $2 \mathrm{~s}$ after immersing the blade in a wind field. The nominal angular velocity of the rotor is 
assumed to be the maximum angular velocity of the ATR rotor $[40]\left(\Omega_{3}^{0}=80 \mathrm{rad} / \mathrm{s}\right)$. To consider a sever wind condition, a uniform vertical wing velocity $V_{3 w}=-10 \mathrm{~m} / \mathrm{s}$ is considered.

Time histories of the blade tip displacement during disengagement with and without actuation control, as well as internal force (without control) at the blade root are depicted in Figs. 6.8-6.10. Comparing Figs. 6.8 and 6.9 shows $17 \%$ reduction in the maximum blade tip displacement with the controller. To analyze the behaviour of the blade during disengagement operation, it can be mentioned that before disengagement, the blade has strong stiffening ( $F_{1}$ or the thick dash line in Fig 6.10). Therefore, aerodynamic and gravity loads cannot deflect the blade. By decreasing the rotor speed, at first because of the weight and the negative lift $\left(F_{3}\right.$ or the solid line in Fig.6.10) blade moves down until it reaches the point where it no longer produces negative lift (at approximately $4.5 \mathrm{~s}$ ). After passing this point, due to the reduction of negative lift the blade goes up until it reaches the point where there is no external force except the blade weight.

A better analysis of the behaviour of the blade during both engagement and disengagement operations is given in the next section where the proposed control strategy is tested on a more accurate BSP dynamic model. However, these two examples show the potential of the proposed strategy to control the blade tip displacements in both constant and variable angular velocities.

\subsection{Controller Effectiveness in the Full BSP Model}

To provide a better evaluation of the proposed controller, its performance has to be assessed in a more accurate BSP model that includes dynamic stall, non-uniform winds, ship motions, and droop/flap stop effects. In Ref. [3], a comprehensive BSP 


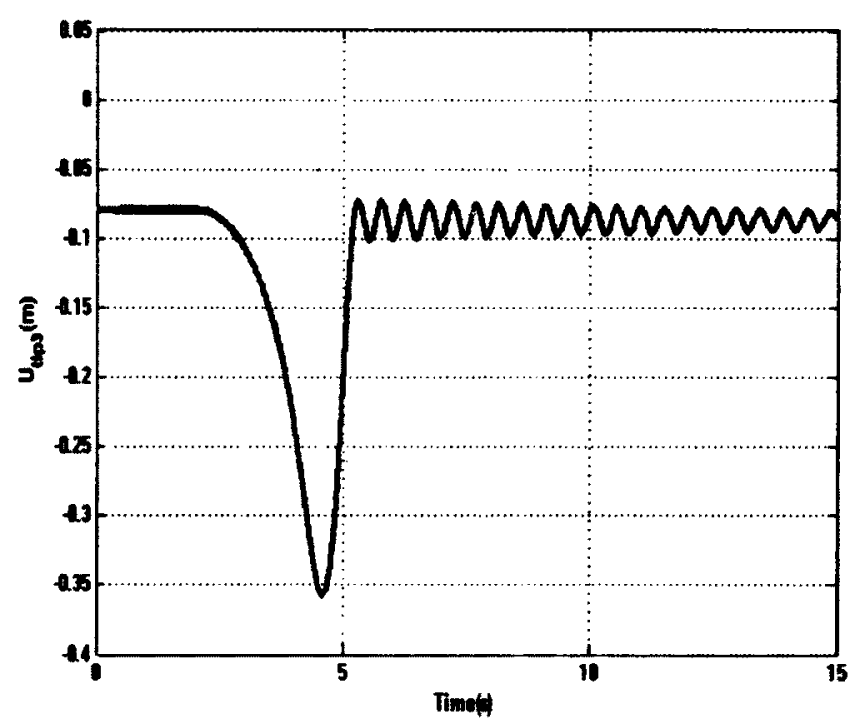

Figure 6.8: Uncontrolled elastic tip displacements of the ATR blade during disengagement operation.

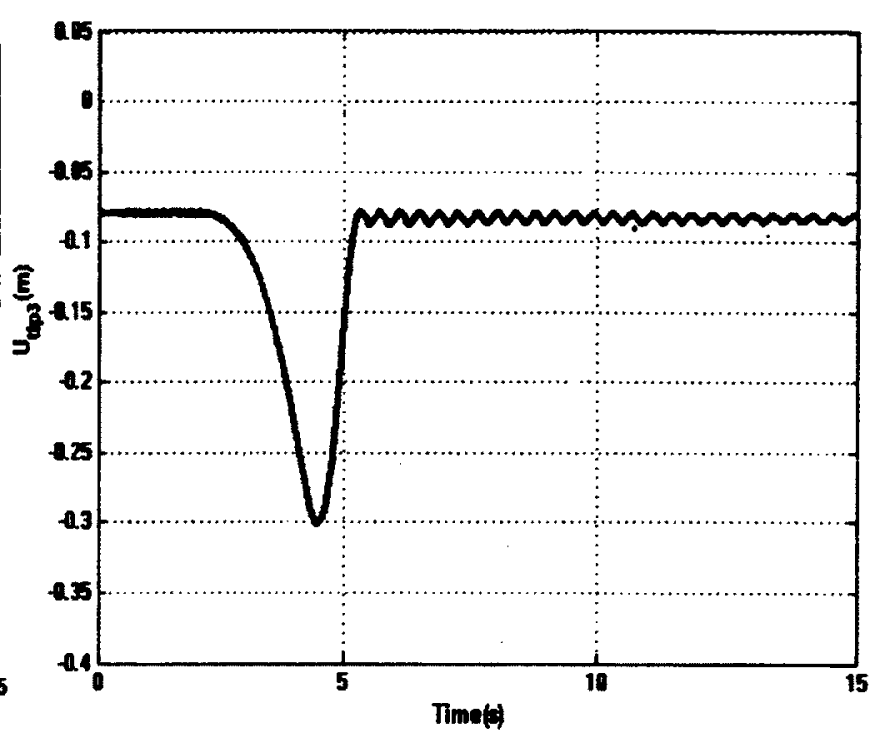

Figure 6.9: Controlled elastic tip displacements of the ATR blade during disengagement operation.

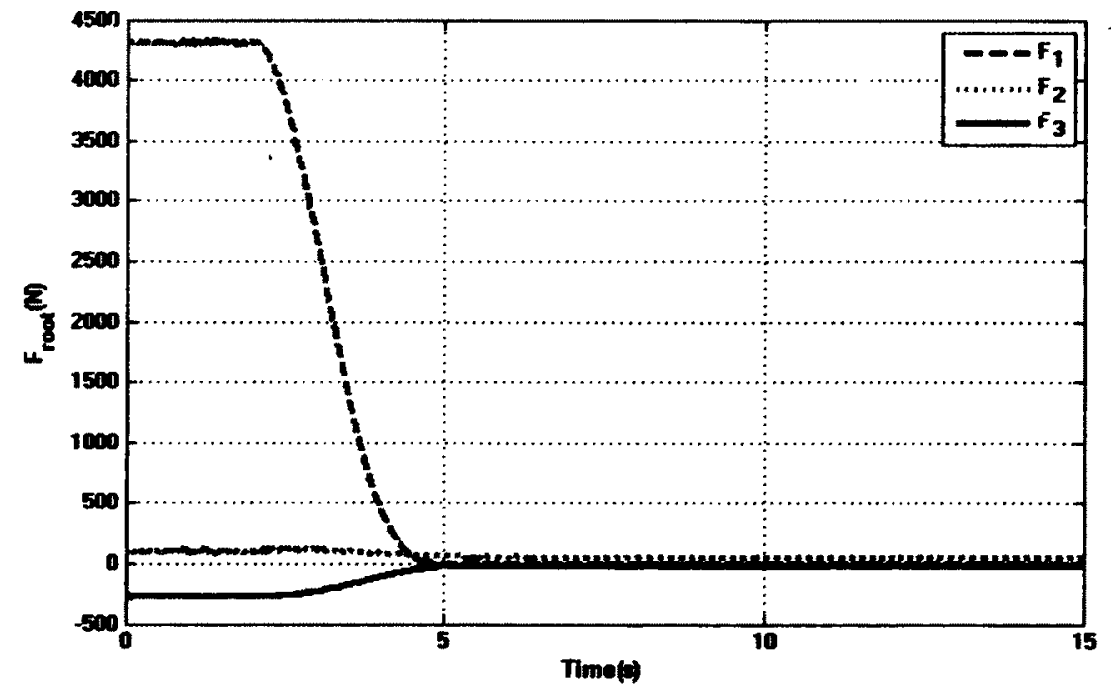

Figure 6.10: Components of internal force at the ATR blade blade root. 
model that has been validated against experimental data and includes different articulation mechanisms and droop/flap stop effects as well as various wind and sea conditions and ship motions was developed. This model is able to capture elastic displacements as well as rigid-body displacements of the blade in a simulated realistic environment. In that reference, a $1 / 6^{\text {th }}$ Mach-scaled integral active twist rotor blade, called IATR, based on the CH-47D helicopter rotor system was adopted for the BSP control. A schematic picture of this rotor system is shown in Fig. 6.11.
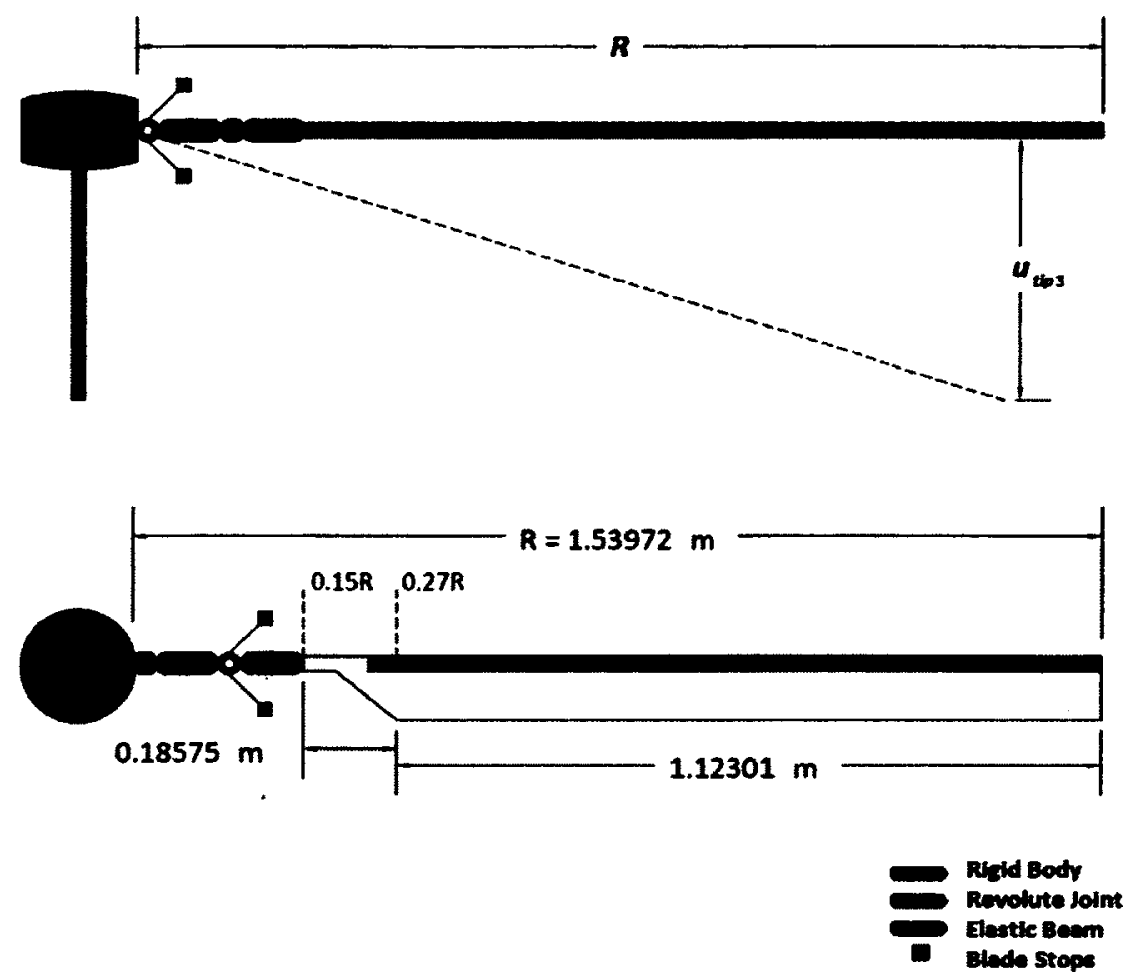

Figure 6.11: Top and front views of IATR blade with typical flap-droop and lead-lag joints and the stops.

The main reason for adopting the integral active twist blade was availability of design data compared to the other active rotor blades. In addition, it was indicated in the literature that while either Mach-scaled or Froude-scaled rotors can be used for helicopter blade aeroelastic analyses, correct actuation requirements for active blades can be obtained by employing Mach-scaled rotors. More information on rotor scaling 
for aeroelastic analyses can be found in ref. [3]. The structural and inertial properties of the IATR in basic SI units are as follows:

$$
\begin{aligned}
& R=10^{-7}\left[\begin{array}{ccc}
2.0567 & 0.0000 & 0.0000 \\
0.0000 & 0.0000 & 0.0000 \\
0.0000 & 0.0000 & 0.0000
\end{array}\right], S=10^{-7}\left[\begin{array}{ccc}
2.7354 & 0.0018 & 22.5295 \\
0.0000 & 0.0000 & 0.0000 \\
0.0000 & 0.0000 & 0.0000
\end{array}\right] \\
& T=10^{-4}\left[\begin{array}{ccc}
476.1213 & 0.0000 & -0.0215 \\
0.0000 & 40.6968 & 0.0000 \\
-0.0215 & 0.0000 & 4.1029
\end{array}\right], G=10^{-1}\left[\begin{array}{ccc}
3.969 & 0.000 & 0.000 \\
0.000 & 3.969 & 0.000 \\
0.000 & 0.000 & 3.969
\end{array}\right] \\
& Q=10^{-4}\left[\begin{array}{ccc}
0.0000 & 0.0000 & -9.9693 \\
0.0000 & 0.0000 & 0.0000 \\
9.9693 & 0.0000 & 0.0000
\end{array}\right], I=10^{-3}\left[\begin{array}{ccc}
0.2769 & 0.0000 & 0.0000 \\
0.0000 & 0.0184 & 0.0000 \\
0.0000 & 0.0000 & 0.2585
\end{array}\right]
\end{aligned}
$$

In the cutoff region, which is between $0.15 \mathrm{R}$ and $0.27 \mathrm{R}$ the structural stiffness and the inertial properties were assumed one and a half times the stiffness and the inertial properties of the rest of blade. The linear initial twist of the blade is -10deg and the blade has a NACA0012 cross-section. Droop/Flap stops are set at $\mp 1$ deg and the lead/lag stops are set at $\pm 12 \mathrm{deg}$. Detailed information about the other properties of the IATR such as lead-lag/flap damping and the collective pitch settings can be found in Ref. [3]. 
In this reference, the actuation voltage was limited to $+1200 \mathrm{~V}$ DC and $\pm 2000 \mathrm{~V}$ AC. These amplitudes are similar to the values applied to the ATR blade reported in Ref. [42]. However, considering the advances in piezoelectric fibres since the advent of the ATR blade, it was decided to use a higher actuation voltage of $\pm 3200 \mathrm{~V}$, which is still providable as mentioned in Ref. [41]. The piezoelectric coefficient vectors $Y^{F}$ and $Y^{M}$ for the IATR blade in basic SI units are as follows:

$$
Y^{F}=10^{-2}\left[\begin{array}{c}
4.106 \\
0.000 \\
0.000
\end{array}\right], \quad Y^{M}=10^{-6}\left[\begin{array}{c}
-245.25 \\
-0.93 \\
-345.66
\end{array}\right]
$$

Given computational speed considerations and the required accuracy, first order 1-D FEM discretization with eleven beam elements was used. The hub maximum nominal speed was considered to be $180 \mathrm{rad} / \mathrm{s}$ and the engagement/disengagement profile similar to Eqs. 3.47 and 3.48 with engagement/disengagement times $t_{\text {eng }}=$ $t_{d i s}=10 / 3 \mathrm{~s}$ was used.

A linear deterministic ship airwake model with a 0.25 gust factor was considered for three seconds before the initiation of engagements/disengagements. The aerodynamic loads were calculated using a nonlinear quasi-steady model with stall effects in different wind speeds and wind over deck (WOD) angles, where the WOD is defined in Fig. 6.12.

Time histories of the blade tip displacement in disengagement operation with wind speed of $45 \mathrm{kn}$ and a WOD angle of $60 \mathrm{deg}$, for two cases with and without feedback control are compared in Figs. 6.13 and 6.14. The corresponding twist angles are compared in Figs. 6.15 and 6.16. Examination of the blade tip displacement in these figures demonstrates the efficiency of the proposed controller for reducing the blade 


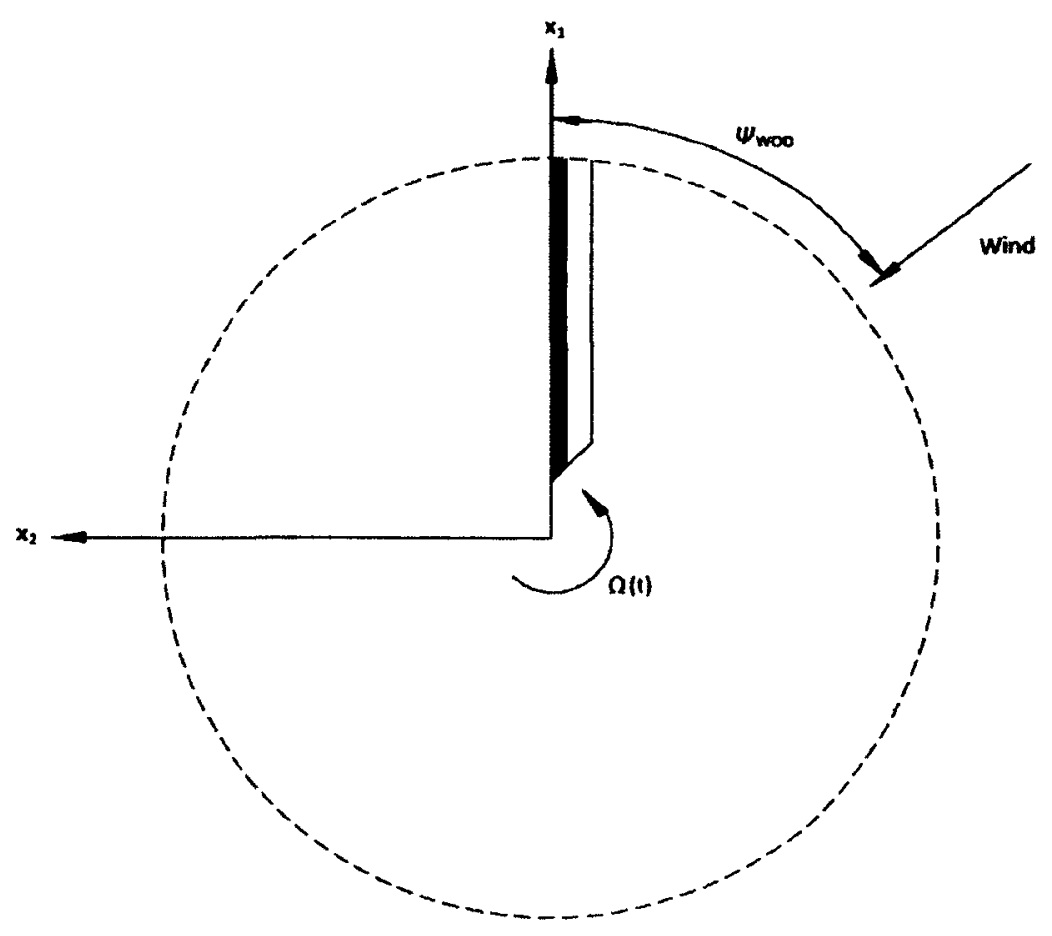

Figure 6.12: Definition of the Wind Over the Deck (WOD) angle.

tip downward displacement up to $25 \%$.

In the beginning, the blade is highly stiffened and does not bend under the aerodynamic loads. By losing its stiffening during the early stages of the BSP, the blade moves down mainly due to gravity loads. However, as it more loses its stiffening the blade moves up mainly due to aerodynamic loads. Comparing time histories of the twist angle in uncontrolled and controlled cases clearly shows the actuation commands effect in increasing the blade twist in order to produce more aerodynamic lift to counter downward displacements. The delta function-like behaviour of the blade around the time $5 \mathrm{~s}$ is due to the sudden reaction of the blade stops that are modelled as a logical switch. However, in reality this reaction would be gradual or at least is not as severe as was simulated.

Similar results about the performance of the proposed control strategy in another 


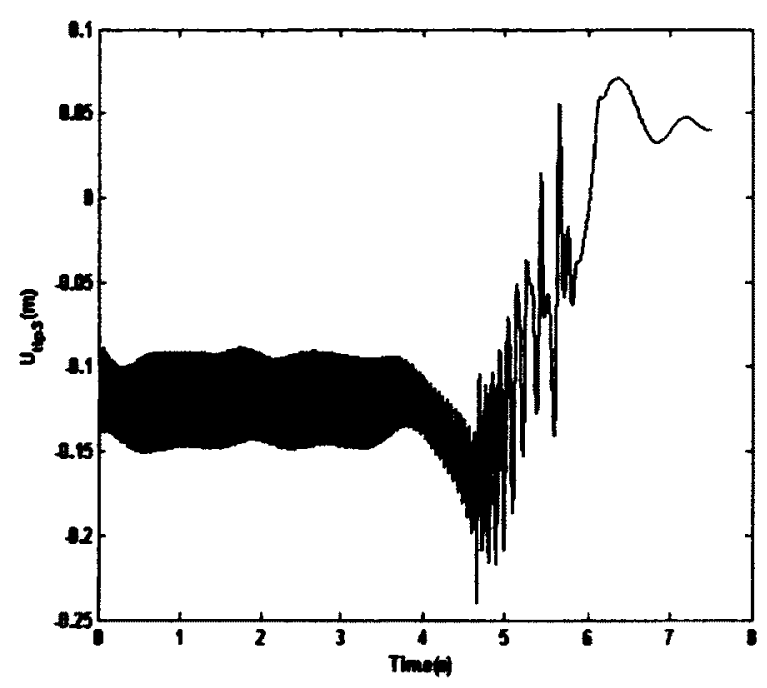

Figure 6.13: Uncontrolled tip displacements of the blade during a disengagement operation, under the wind conditions of $45 \mathrm{kn}$ and $60^{\circ} W O D$, including ship motion.

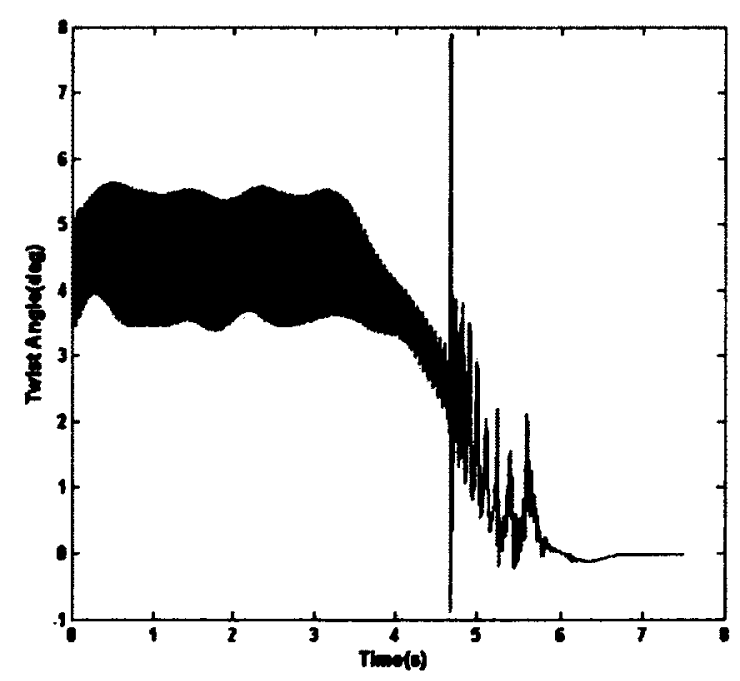

Figure 6.15: Uncontrolled twist angle of the blade during a disengagement operation, under the wind conditions of $45 \mathrm{kn}$ and $60^{\circ} W O D$, including ship motion.

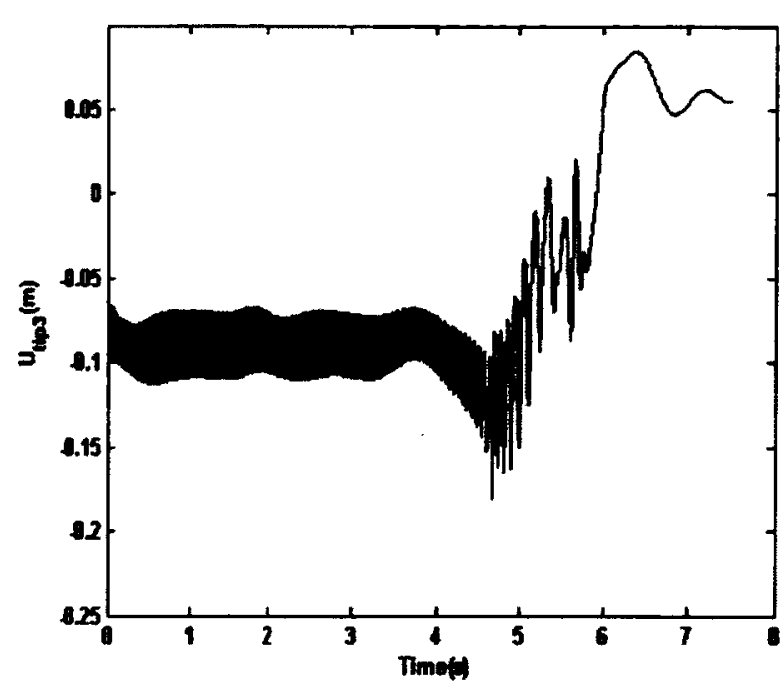

Figure 6.14: Controlled tip displacements of the blade during a disengagement operation, under the wind conditions of $45 \mathrm{kn}$ and $60^{\circ} W O D$, including ship motion.

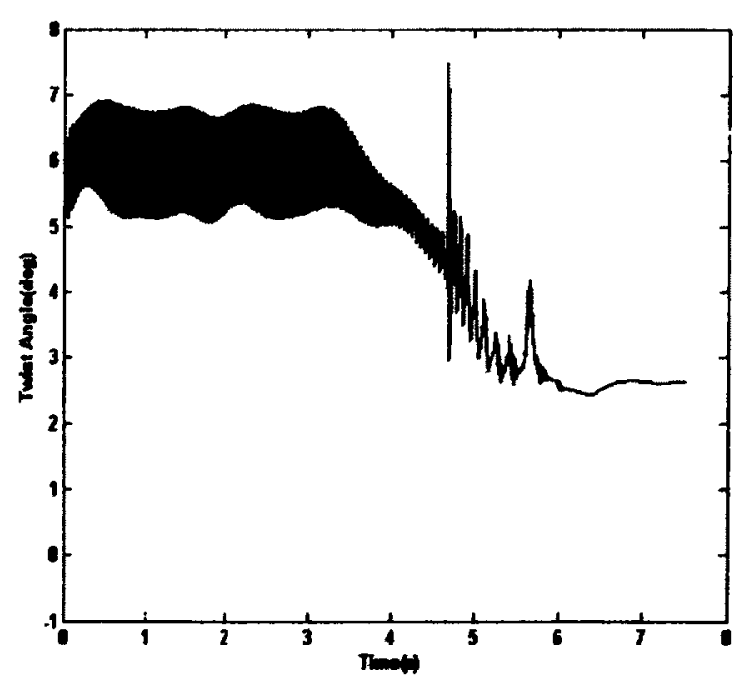

Figure 6.16: Controlled twist angle of the blade during a disengagement operation, under the wind conditions of $45 \mathrm{kn}$ and $60^{\circ} W O D$, including ship motion. 


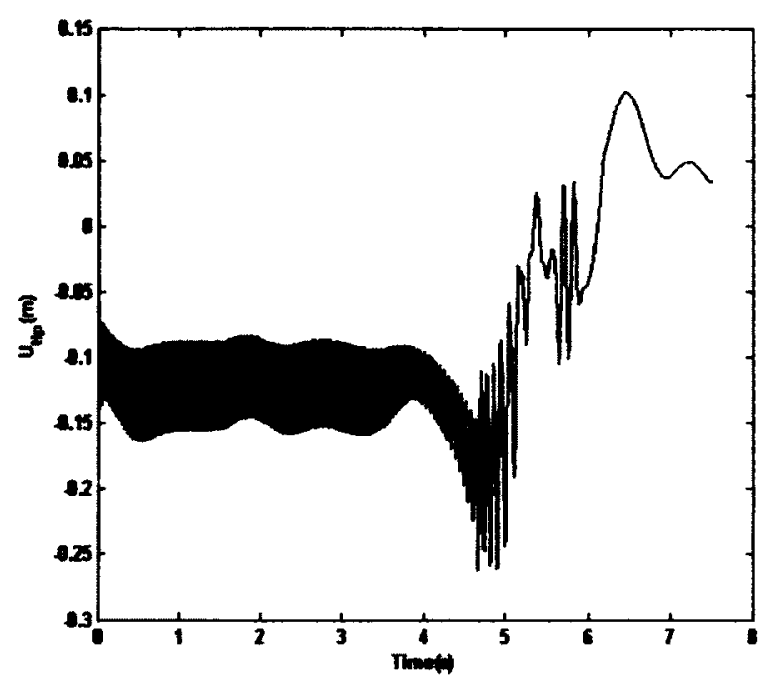

Figure 6.17: Uncontrolled tip displacements of the blade during a disengagement operation, under the wind conditions of $50 \mathrm{kn}$ and $0^{\circ} W O D$, including ship motion.

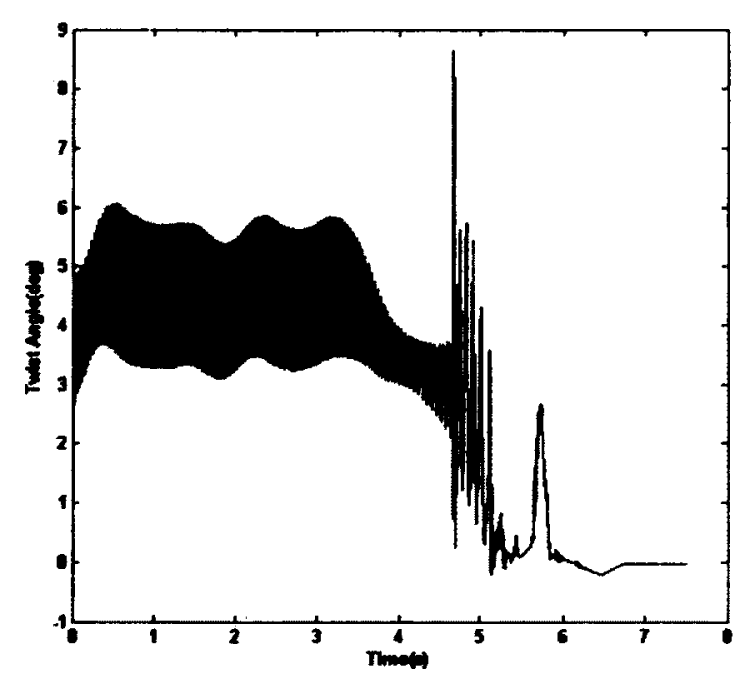

Figure 6.19: Uncontrolled twist angle of the blade during a disengagement operation, under the wind conditions of $50 \mathrm{kn}$ and $0^{\circ} W O D$, including ship motion.

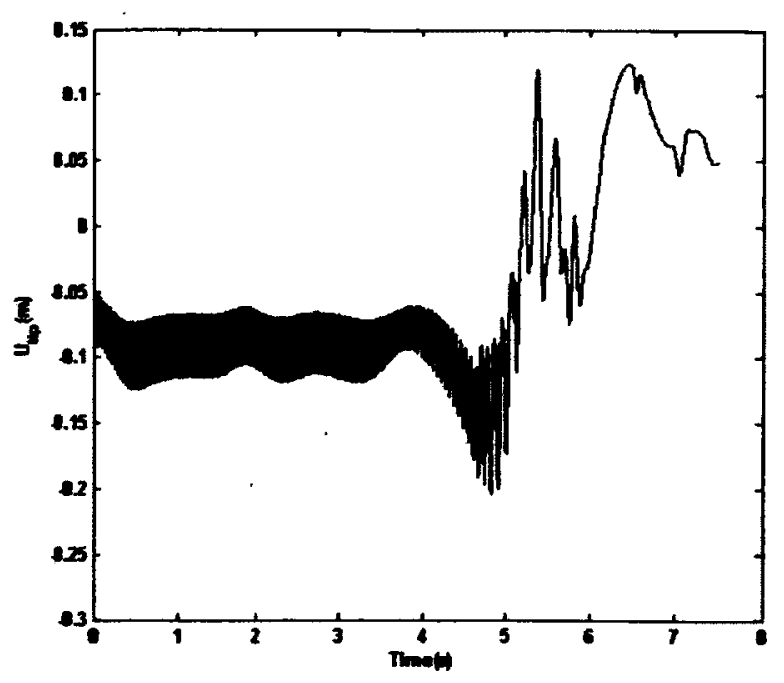

Figure 6.18: Controlled tip displacements of the blade during a disengagement operation, under the wind conditions of $50 \mathrm{kn}$ and $0^{\circ} W O D$, including ship motion.

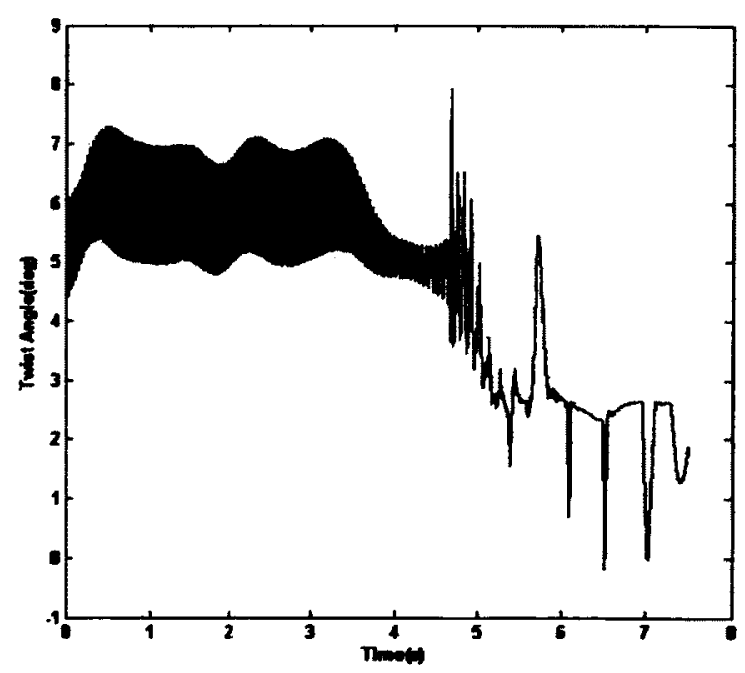

Figure 6.20: Controlled twist angle of the blade during a disengagement operation, under the wind conditions of $50 \mathrm{kn}$ and $0^{\circ} W O D$, including ship motion. 
disengagement operation can be obtained by comparing time histories of the tip displacement and the twist angle during the disengagement operation with a wind speed of $50 \mathrm{kn}$, WOD angle of $0 \mathrm{deg}$, a ship speed of $10 \mathrm{kn}$, and a ship heading angle $0 \mathrm{deg}$ that are shown in Figs. 6.17-6.20. In this case, a $27 \%$ reduction in the blade tip displacement is obtained through feedback control.

The performance of the proposed control strategy during the engagement operation is evaluated by comparing the blade tip displacement in two controlled and uncontrolled conditions. In Ref. [3], it was realized that a wind speed of $60 \mathrm{kn}$ with a WOD angle of $270 \mathrm{deg}$ can cause one of the largest blade tip displacements. Therefore, this condition was chosen to assess the performance of the controller. Figs. 6.21 and 6.22 compare time histories of the blade tip displacement in this environmental condition in the absence of ship motion, for both uncontrolled and controlled cases. The corresponding twist angles are compared in Figs. 6.23 and 6.24. As can be seen, considerable reduction (up to $30 \%$ ) in the blade downward tip displacement can be obtained using the proposed controller.

The effect of ship motion on the performance of the controller is investigated by considering a wind speed of $50 \mathrm{kn}$, ship speed of $10 \mathrm{kn}$ and a ship heading angle of $60 \mathrm{deg}$ with other parameters unchanged from the previous case. Figs. 6.25 and 6.26 show that even in the presence of the ship motion, a downward tip displacement reduction up to $21 \%$ is achievable through application of the current controller,

A further assessment of the proposed control strategy during the engagement operation is obtained by testing another case in which the wind speed of $50 \mathrm{kn}$ with WOD angle of $180 \mathrm{deg}$ is applied to the blade of a helicopter landed on a ship. with a speed of $10 \mathrm{kn}$ and a heading angle of $0 \mathrm{deg}$. Figs. 6.27-6.30 show the results obtained using the proposed control strategy. Again, up to $30 \%$ reduction in blade tip downward displacement is obtained using the controller.

It was also observed that the proposed strategy in some cases has increased the 


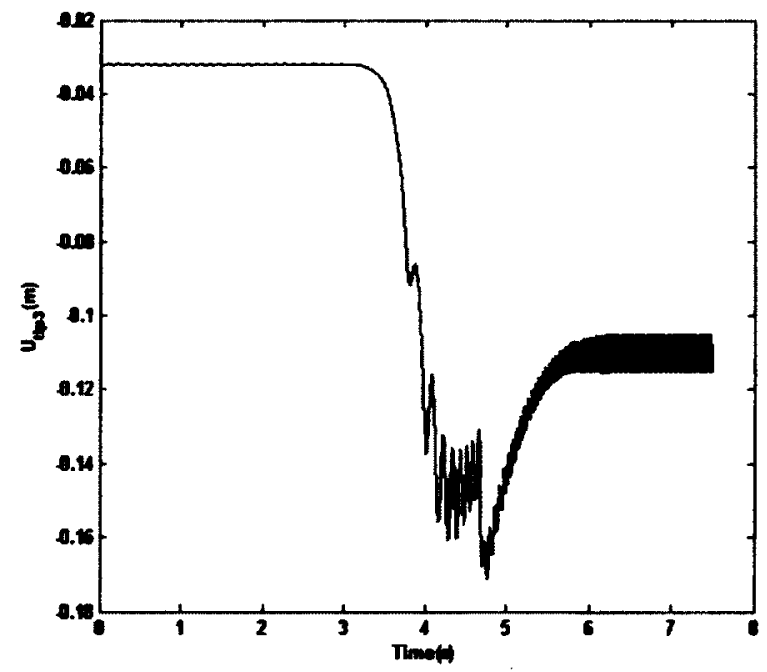

Figure 6.21: Uncontrolled tip displacements of the blade during an engagement operation, under the wind conditions of $60 \mathrm{kn}$ and $270^{\circ}$ WOD.

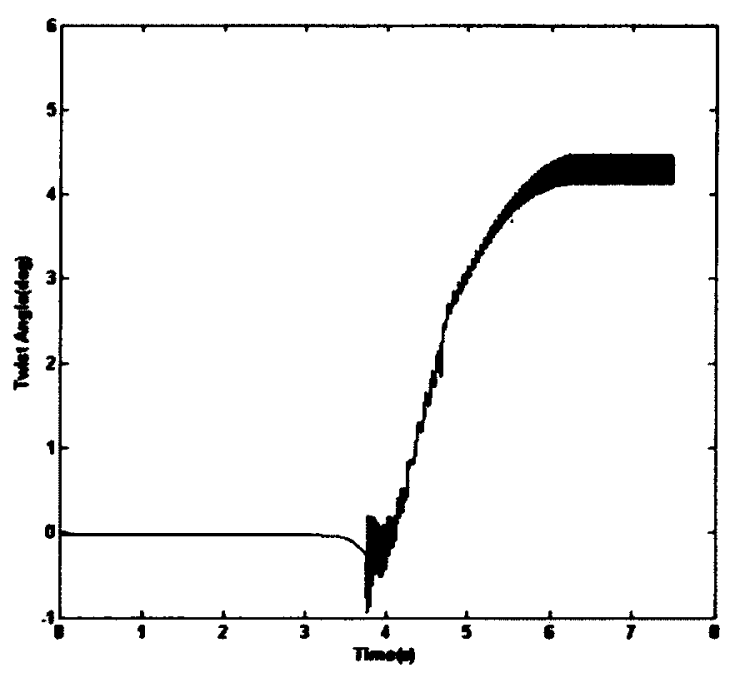

Figure 6.23: Uncontrolled twist angle of the blade during an engagement operation, under the wind conditions of $60 \mathrm{kn}$ and $270^{\circ}$ WOD.

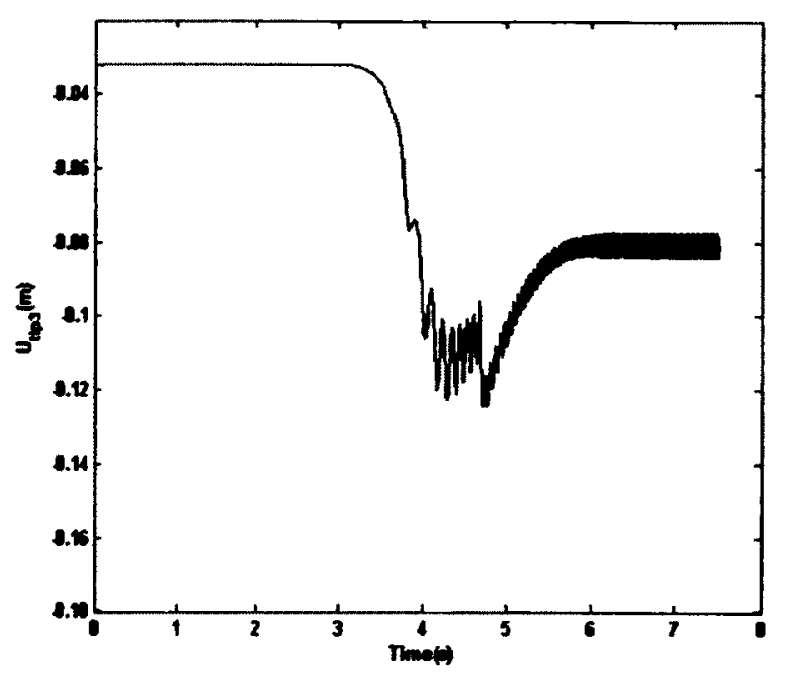

Figure 6.22: Controlled tip displacements of the blade during an engagement operation, under the wind conditions of $60 \mathrm{kn}$ and $270^{\circ}$ WOD.

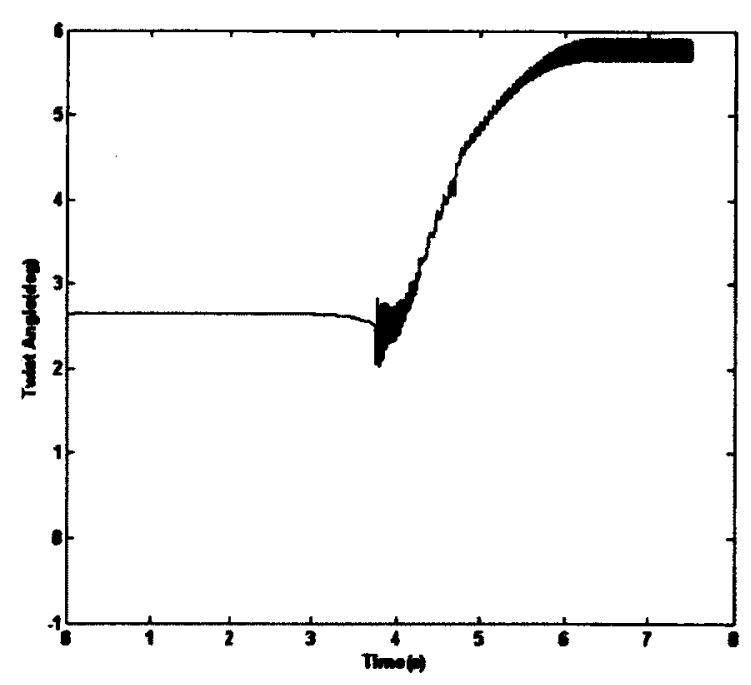

Figure 6.24: Controlled twist angle of the blade during an engagement operation, under the wind conditions of $60 \mathrm{kn}$ and $270^{\circ} W O D$ 


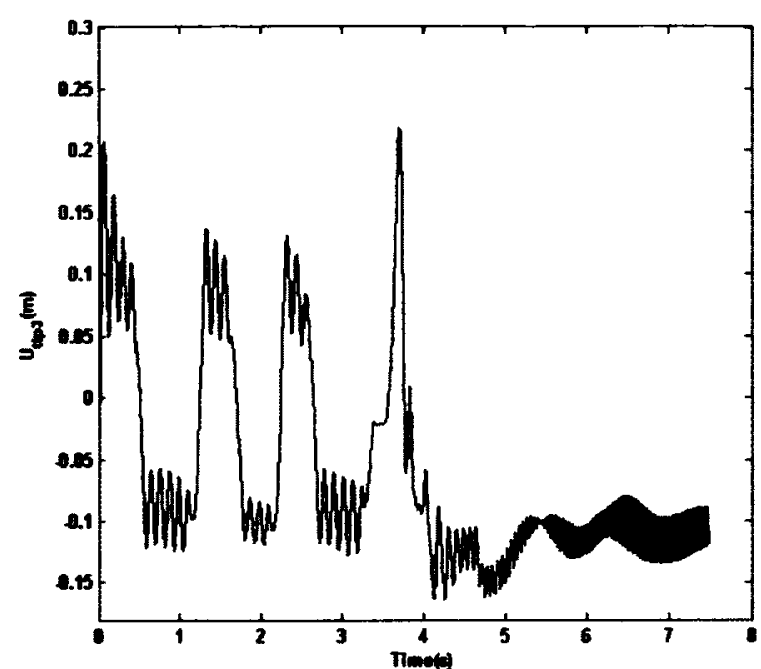

Figure 6.25: Uncontrolled tip displacements of the blade during an engagement operation, under the wind conditions of $50 \mathrm{kn}$ and $270^{\circ} W O D$, including ship motion.

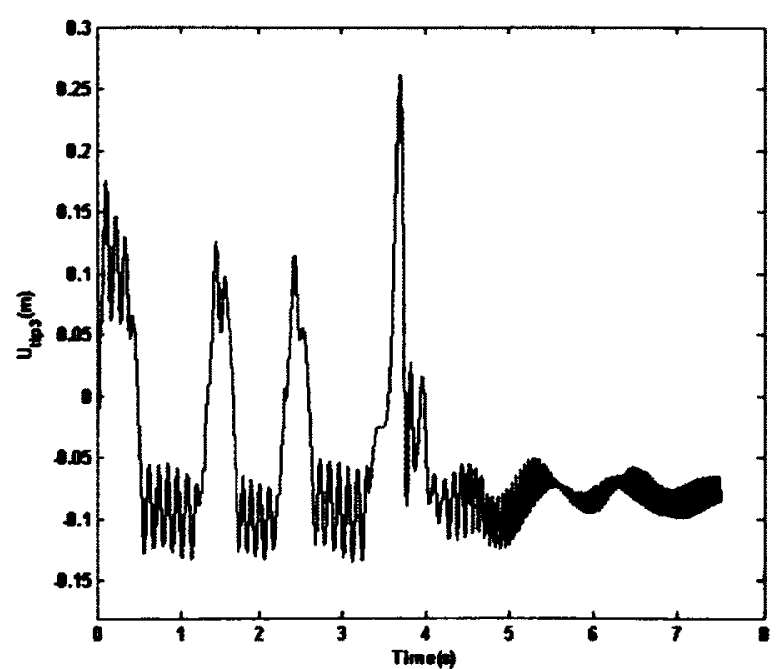

Figure 6.26: Controlled tip displacements of the blade during engagement operation, under the wind conditions of $50 \mathrm{kn}$ and $270^{\circ} W O D$, including ship motion.

blade tip upward displacement. This increase in upward displacement is not important in the case of BSP analysis where the whole objective is to increase the blade tip clearance from the ship deck or helicopter fuselage/tailboom.

The proposed control strategy has shown its effectiveness in countering the BSP in the most severe wind and sea conditions associated with shipboard helicopter flight operations. The developed controller induces strains in the blade to change its twist to counter the aerodynamic loads, and thereby it changes the structural behaviour of the blade and controls its structural displacements. As recommended in the next chapter, further actuation strategies and modelling improvements can be considered to design a BSP controller with better performance and robustness properties. 


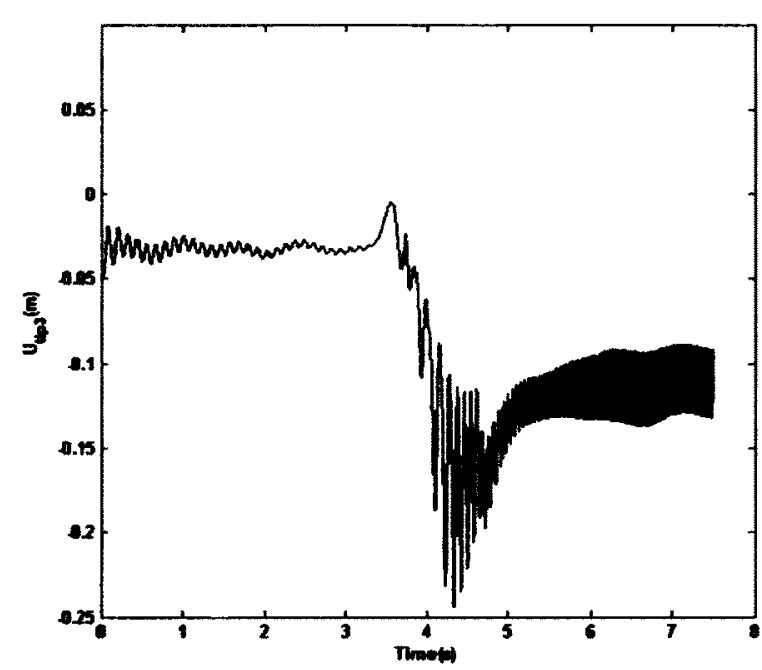

Figure 6.27: Uncontrolled tip displacements of the blade during an engagement operation, under the wind conditions of $50 \mathrm{kn}$ and $180^{\circ} W O D$, including ship motion.

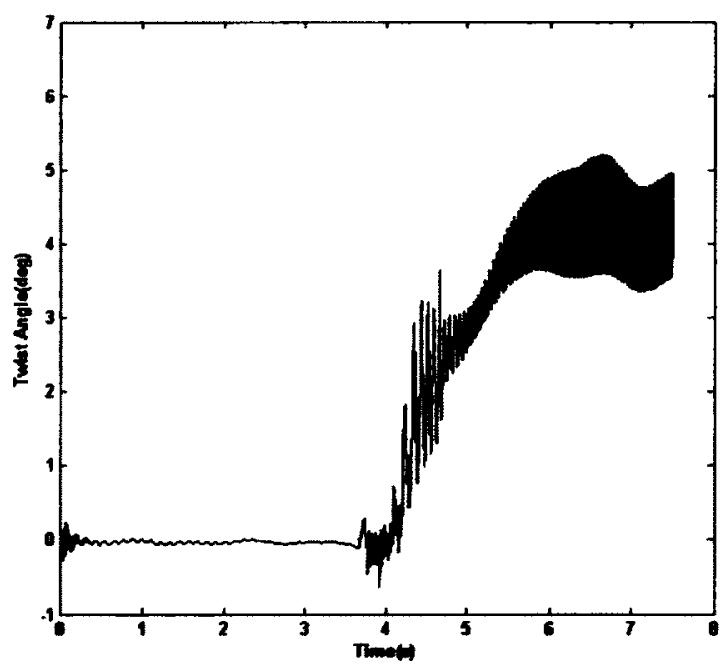

Figure 6.29: Uncontrolled twist angle of the blade during an engagement operation, under the wind conditions of $50 \mathrm{kn}$ and $180^{\circ} W O D$, including ship motion.

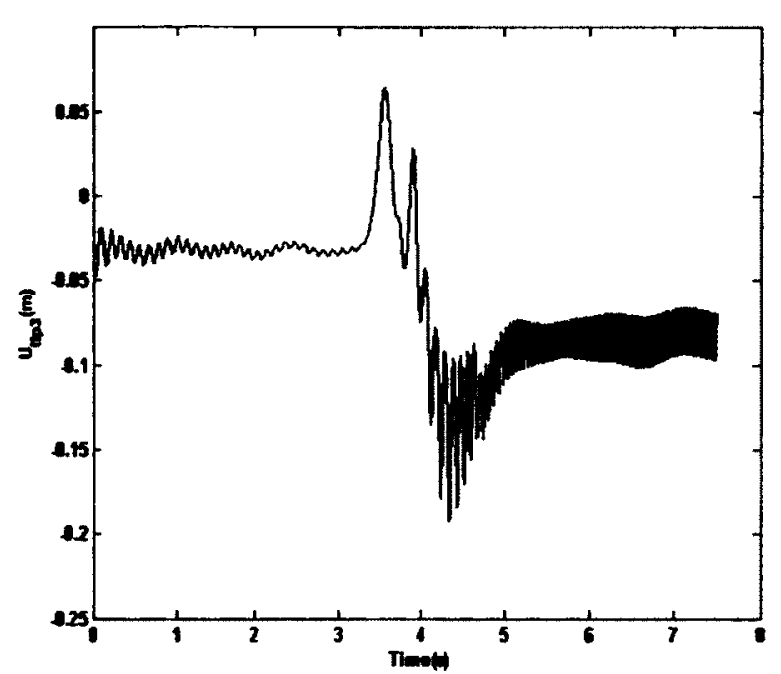

Figure 6.28: Controlled tip displacements of the blade during an engagement operation, under the wind conditions of $50 \mathrm{kn}$ and $180^{\circ} W O D$, including ship motion.

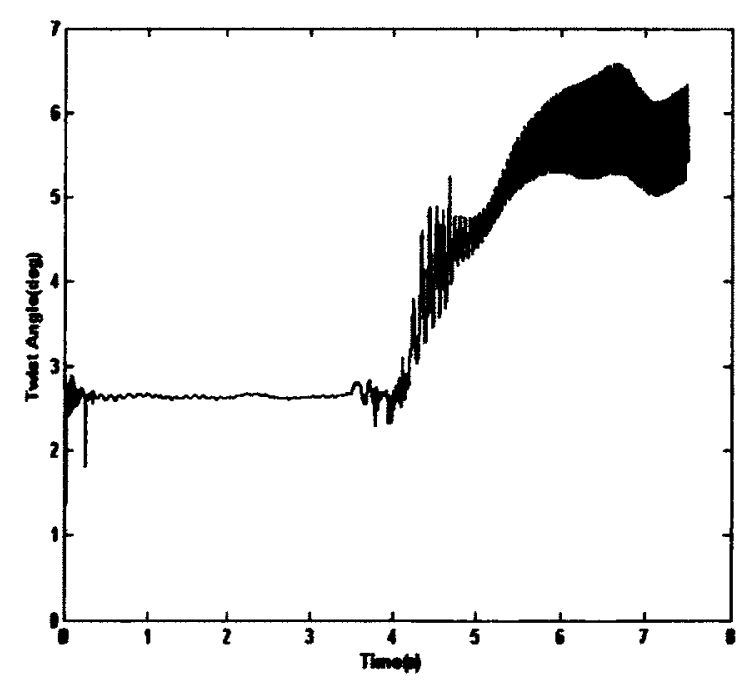

Figure 6.30: Controlled twist angle of the blade during engagement operation, under the wind conditions of $50 \mathrm{kn}$ and $270^{\circ} W O D$, including ship motion. 


\section{Chapter 7}

\section{Discussion and Conclusion}

\subsection{Summary}

In this research, a feedback control system based on a reduced, yet reliable, model of a helicopter aeroelastic phenomenon occurring during maritime helicopter rotor engagement/disengagement operations, known as Blade Sailing Phenomenon (BSP), was developed. To obtain a reliable reduced model capable of meeting the feedback control design requirements, a set of state-space equations, modelling different factors contributing to the occurrence of the phenomenon was established.

The Unified Airloads Model that has been developing over decades was employed to capture the linear unsteady generalized aerodynamic loads on the blade. Basic elements of this model were introduced and detailed derivations resulted in obtaining and defining the lift, drag, and pitching moment equations for the blade in a statespace form.

The nonlinear structural behaviour of the blade was modelled through geometrically-exact beam intrinsic equations. These equations were shown to be desirable for control purposes due to their analytical form and compactness.

Integrally Actuated Twist (IAT) was chosen as an actuation strategy. In IAT, actively controlled induced strains twist the blade in order to modify the aerodynamic 
loads. Along with aerodynamic loads, gravity, wind, and actuation effects were incorporated in an intrinsic formulation and the resulting equations were used to design an energy-based feedback controller.

The total energy of the blade was obtained by applying appropriate weighting functions to each equation, linking the complete system dynamics to one equation: the law of conservation of energy. Defining the deviation of the blade from an ideal zero displacement condition, the deviated variables, and thereby deviated energy of the blade were obtained. Deviated energy was used as a candidate Lyapunov function, and the control signals were found such that the candidate Lyapunov function became a deviation minimizing function.

To evaluate the designed controller, the Galekin Spectral Discretization Method was used to discretize the reduced dynamical model of the BSP in the space domain. The accuracy of the Galerkin discretization was validated by examples capturing the structural behaviour of a beam and satisfying the boundary and constraint values. The designed controller was then examined on a Galerkin discretized blade dynamics. Fixed and varying rotor speeds were considered in a series of simulations. Simulations showed considerable reduction in the blade downward displacement through proposed feedback control and therefore proved the potential of the proposed control strategy for BSP control.

To evaluate the controller in a more realistic BSP environment, a comprehensive BSP model that was previously validated against experimental data was used as a test-bed. The controller was tested for engagement/disengagement operations in different wind and sea conditions. Results showed significant reduction in the blade tip displacement through application of the proposed feedback controller. 


\subsection{Contributions}

The main contributions of this research can be categorized in three parts: BSP modelling, Blade Galerkin discretization, and BSP control.

- BSP modelling A control-centric model that presents a compact set of equations suitable for feedback control was developed in this research. While other BSP models are either prediction-oriented and as a result too complex for controller design or control-oriented and therefore too simple to accurately predict the real BSP, the current BSP model provides a compromise solution.

- Blade discretization A Galerkin discretization of the blade intrinsic equations, covering the gravity and wind loads as well unsteady aerodynamic effects, was carried out in this research. Previous works in this regard do not include the unsteady aerodynamic effects, the blade gravity loads, and intrinsic structural damping loads.

- BSP control A nonlinear Lyapunov feedback controller was designed and tested on a comprehensive BSP model. The application of the Lyapunov stability theorem to the BSP control problem is novel and testing a feedback controller on a comprehensive BSP model has not been performed previously.

\subsection{Future work}

This research can have many extensions in different areas of the aeroservoelasticity applied to low RPM helicopter blades. The most obvious extension is to build an active composite rotor blade model with embedded actuators and sensors to evaluate the proposed control strategy in a set of experiments. The following list enumerates other possible extensions: 
- Aerodynamic modelling The current model does not take into account the nonlinear aerodynamic behavior of the stall and the dynamic stall of the blade in the Unified Airloads Model. Adding these nonlinearities is inevitable if one needs to use this aerodynamic model for the BSP with the ultimate objective of developing a prediction-oriented BSP model. In this regard, all necessary equations are given in Chapter 2. The Unified Airloads Model is able to predict the aerodynamic loads on flexible airfoils. This capability gives a great opportunity to a control designer to design and test different morphing-based actuation strategies such as active camber and leading-edge/trailing-edge dynamic droops.

- Wind effects modelling The control-centric BSP model developed in this research does not include non-uniform wind loads on the blade. Initial provisions are given in Chapter 3. Section 3.3.5. However, to add the wind effect to the final energy balance equation, one needs to find appropriate weighting functions.

- Galerkin discretization In this research, the conventional Galerkin method in which identical comparison and weighting functions are used for discretization is employed. It is reported in some literature, that similar comparison and weighting functions can cause solution instability problems [36]. Therefore, a potential improvement to the current work is to apply dissimilar comparison and weighting functions (modern Galerkin method). In addition, Legendre polynomials, which are used as comparison and weighting functions in this research, have a slow rate of convergence. Therefore, it is definitely worth trying different polynomials such as Chebyshev polynomials.

- Control design The current Lyapunov controller is designed to control the structural displacements of the blade tip. In the actual BSP, the total displacement of the blade is composed of both structural and rigid-body displacements. 
The current BSP control can be extended to include those rigid-body displacements, as well. In this case, it seems to be worth investigating other actuation and control strategies such as adding rigid control surfaces to the blade or swashplate control. They may present more controllability over the BSP.

- Programming In terms of computational efficiency, one major drawback of the current discretized model is its slow convergence. In addition to Legendre polynomials used for discretization, this slow convergence can be attributed to two other factors:

First, to capture the real behaviour of the blade, a sufficient number of comparison functions have to be included. Due to the fast variation of the structural high-frequency modes, small solution time steps are required for convergence. This problem is mainly rooted in the mathematics of the Galerkin discretization method and as such is unavoidable.

Second, to use the features already present in the MATLAB software and SIMULINK environment, and for simplicity in programming, especially to do tensor calculations, the current computer code is written in MATLAB; And therefore is not time efficient. One suggestion to resolve this problem is to rewrite the code in a faster computational environment such as $\mathrm{C}++$ or MATLAB Real-Time. This modification will be inevitable when it comes to the experiments and the real-time implementation of the controller. In this regard, it should be noted that the current version of the MATLAB Real-Time environment does not support the tensor calculations used in this work. 


\section{List of References}

[1] S. J. Newman. "The phenomenon of helicopter rotor blade sailing." Joumal of Aerospace Engineering 213(6) (1999).

[2] C. K. Leong. "Challenges associated with flight deck operations." FOCUS, RSAF Safety Magazine 61 (2009).

[3] F. Khouli. Modelling and Attenuation Feasibility of the Aeroelastic Response of Active Helicopter Rotor Systems during the Engagement/Disengagement Phase of Maritime Operation. Ph.D. thesis, Ottawa-Carleton Institute for Mechanical and Aerospace Engineering, Carleton University (2009).

[4] A. Wall. A Discrete Approach to Modelling Helicopter Blade Sailing. Ph.D. thesis, Ottawa-Carleton Institute for Mechanical and Aerospace Engineering, Carleton University (2009).

[5] W. P. J. Geyer and E. C. Smith. "Aeroelastic analysis of transient blade dynamics during shipboard engage/disengage operations." Journal of Aircraf 35 (1995).

[6] J. Keller. Analysis and Control of the Transient Aeroelastic Response of Rotors during Shipboard Engagement and Disengagement Operations. Ph.D. thesis, The Pennsylvania State University (2001).

[7] B. van del Wall and J. Leishman. "On the influence of time-varying flow velocity on unsteady aerodynamics." Journal of American Helicopter Society 39 (1994).

[8] J. G. Leishman and T. Beddoes. "A semi-empirical model for dynamic stall." Journal of the American Helicopter Society (1989).

[9] D. Peters and A. S. D. Barwey. "An integrated airloads-inflow model for use in rotor aeroelasticity and control analysis." Mathematical and Computer Modelling 19(3/4), 109-123 (1994). 
[10] D. Peters and C. He. "Finite-state induced flow models, part ii: Threedimensional rotor disk." Journal of Aircraft 32(2) (1995).

[11] D. Peters, S. Karunamoorthy, and W. Cao. "Finite state induced flow models part i: Two dimensional thin airfoil." Journal of Aircraft 32(2), 313-322 (1995).

[12] D. Peters, M. A. Hsiegh, and A. Torreto. "A state-space airloads theory for flexible airfoils." Journal of the American Helicopter Society August, 329-342 (2007).

[13] L. A. Ahaus and D. Peters. "Unified airloads model for morphing airfoils in dynamic stall." In "Proceedings of the American Helicopter Society Specialist's Conference on Aeromechanics," San Francisco, California (2010).

[14] L. A. Ahaus, D. A. Peters, and A. J. Kahler. "Simplified analysis of sectional airloads on morphing airfoils." In "Proceedings of the American Helicopter Society 66th Annual Forum," (2010).

[15] S. J. Newman. "A theroetical model for predicting the blade sailing behavior of a semi-rigid rotor helicopter." Vertica 12 (1990).

[16] J. A. Keller and E. C. Smith. "Analysis and control of the transient shipboard engagement behavior of rotor systems." In "Proceedings of the American Helicopter Society 55th Annual Forum," American Helicopter Society (1999).

[17] C. L. Bottasso and O. A. Bachau. "Multibody modelling of engage and disengage operations of helicopter rotors." Journal of American Helicopter Society 46, 290-300 (2001).

[18] O. A. Bachau, C. L. Bottasso, and Y. G. Nikishkov. "Modeling rotorcraft dynamics with finite element multibody procedures." Mathematical and Computer Modelling 33, 1113-1137 (2001).

[19] M. P. Jones and S. J. Newman. "A method of reducing blade sailing through the use of trailing edge flaps." In "Proceedings of the American Helicopter Society 63rd Annual Forum," American Helicopter Society (2007).

[20] V. Piccirillo, K. A. L. Casti, R. L. da Cunha Barroso Ramos, and L. C. S. Goes. "Aeroelastic control of helicopter blade sailing in unsteady flow using smart materials." (2009). 
[21] V. Piccirillo., L. C. S. Goes, R. L. da Cunha Barroso Ramos, and J. M. Balthazar. "Application of predictive control techniques to a helicopter blade sailing system." (2010).

[22] R. L. da Cunha Barroso Ramos, D. de Andrade, and L. C. S. Ges. "Individual blade root control of helicopter blade sailing for articulated shipboard rotors." In "Proceedings of the American Helicopter Society 65th Annual Forum," American Helicopter Society (AHS) (2009).

[23] R. L. da Cunha Barroso Ramos, D. de Andrade, and L. C. S. Ges. "Performance analysis of a helicopter blade sailing aeroservoelastic system with individual blade root control and reverse flow effects." In "Proceedings of the 11th Pan-American Congress of Applied Mechanics," PACAM (2010).

[24] D. H. Hodges. "Geometrically exact, intrinsic theory for dynamics of curved and twisted anisotropic beams." AIAA Journal 41 (2003).

[25] M. Althoff. Nonlinear Dynamics and Control of Integrally Actuated Helicopter Blades. Master's thesis, Virginia Polytechnic Institute and State University (2005).

[26] D. Petot. "Progress in the semi-empirical prediction of the aerodynamic forces due to large amplitude oscillations of an airfoil in attached or separated flow." In "Proceedings of the Ninth European Rotorcraft Forum," Stresa, Italy (1983).

[27] M. H. Hansen, M. Gaunaa, and H. A. Madsen. "A beddoes-leishman type dynamic stall model in state-space and indicial formulations." Technical report, Riso National Laboratory, Roskilde, Denmark, Roskilde, Denmark (2004).

[28] D. H. Hodges. Nonlinear Composite Beam Theory, volume 213. American Institue of Aeronautics and Astronautics(AIAA) (2006).

[29] M. L. Wilbur, P. H. Mirick, W. T. Yeager, and C. W. Langston. "Vibratory loads reduction testing of the [nasa/army/mit] active twist rotor." In "Proceedings of the American Helicopter Society 57th Annual Forum," (2001).

[30] S.-B. Choi and Y.-M. Han. Piezoelectric Actuators, Control Applications of Smart Materials. CRC.Press (2010).

[31] M. J. Patil and D. H. Hodges. "Flight dynamics of highly flexible flying wings." Journal of Air 43(6) (2006).

[32] H. K. Khalil. Nonlinear Systems. Prentice Hall, third edition (2002). 
[33] S. Sastry. Nonlinear Systems, Analysis, Stability, and Control. Springer (1999).

[34] J. E. Slotine and W. Li. Applied Nonlinear Control. Prentice Hall (1991).

[35] J. Shen, T. Tang, and L.-L. Wang. Spectral Methods, Algorithms, Analysis and Applications. Springer (2011).

[36] C. A. J. Fletcher. Computational Galerkin Methods. Springer (1984).

[37] K. H. Huebner, D. L. Dewhirst, D. E. Smith, and T. G. Byrom. The Finite Element Method for Engineers. Fourth edition (2001).

[38] M. Abramowitz and I. A. Stegun. Handbook of Mathematical Functions with Formulas, Graphs, and Mathematical Tables. Ninth edition (1970).

[39] M. V. Soare, P. P. Teudorescu, and I. Toma. Ordinary Differential Equations with Applications to Mechanics. Springer (2007).

[40] J. P. Traugott, M. J. Patil, and F. Holzapfel. "Nonlinear dynamics and control of integrally actuated helicopter blades." In "Proceedings of the 13th Adaptive Structures Conference," Ameriacan Institute of aeronautics and Astronautics (AIAA) (2005).

[41] J. P. Rodgers. Development of an Integral Twist-Actuated Rotor Blade for Individual Blade Control. Ph.D. thesis, Massachusetts Institute of Technology (MIT) (1999).

[42] C. E. S. Cesnik, S. Shin, and M. L. Wilbur. "Dynamic response of active twist rotor blades." Smart Materials and Structures 10, 62-76 (2001). 


\section{Appendix A}

\section{Aerodynamic Modelling}

\section{A.1 Unstalled Generalized Loads}

Vectors $\left\{h_{n}\right\},\left\{v_{n}\right\},\left\{\lambda_{0}\right\},\left\{\lambda_{1}\right\}$, and $\{1\}$, and matrices $[K],[C],[G],[S],[H]$ and $[M]$ in Eqs 2.1-2.3 have the dimensions $(M+1) \times 1$ and $(M+1) \times(M+1)$, respectively, and are defined below:

$\left\{h_{n}\right\}=\left\{\begin{array}{c}h_{0} \\ h_{1} \\ \cdot \\ \cdot \\ v_{1} \\ \cdot \\ h_{M}\end{array}\right\},\left\{v_{n}\right\}=\left\{\begin{array}{c}v_{0} \\ 0 \\ \cdot \\ 0\end{array}\right\},\left\{\lambda_{0}\right\}=\left\{\begin{array}{c}\lambda_{0} \\ 0 \\ \cdot \\ 0\end{array}\right\},\left\{\lambda_{1}\right\}=\left\{\begin{array}{c}\lambda_{0} \\ \lambda_{1} \\ 0 \\ \cdot \\ \cdot \\ 0\end{array}\right\},\{1\}=\left\{\begin{array}{l}1 \\ 0 \\ \cdot \\ 0\end{array}\right\}$ 


$$
\begin{aligned}
& {[K]=\left[\begin{array}{ccccccc}
0 & f & 3 & 3 f & 4 & 5 f & \cdots \\
0 & -1 / 2 & 0 & 0 & 0 & 0 & \cdots \\
0 & 0 & -2 / 2 & 0 & 0 & 0 & \ddots \\
0 & 0 & 0 & -3 / 2 & 0 & 0 & \ddots \\
0 & 0 & 0 & 0 & -4 / 2 & 0 & \ddots \\
\vdots & \vdots & \vdots & \vdots & \vdots & \ddots & \ddots
\end{array}\right]} \\
& {[S]=\left[\begin{array}{cccc}
f & 0 & 0 & \ldots \\
0 & 0 & 0 & \ldots \\
0 & 0 & 0 & \ldots \\
\vdots & \vdots & \vdots & \ddots
\end{array}\right]} \\
& {\left[\begin{array}{lllllll}
f & 1 & 0 & 0 & 0 & \cdots
\end{array}\right]} \\
& \begin{array}{llllll}
-1 / 2 & 0 & 1 / 2 & 0 & 0 & \ldots
\end{array} \\
& {[C]=\left[\begin{array}{cccccc}
0 & -1 / 2 & 0 & 1 / 2 & 0 & \ddots \\
0 & 0 & -1 / 2 & 0 & 1 / 2 & \ddots
\end{array}\right.} \\
& \begin{array}{llllll}
0 & 0 & 0 & -1 / 2 & \ddots & \ddots
\end{array} \\
& \vdots \quad \vdots \quad \vdots \quad \vdots \quad \vdots \quad \ddots
\end{aligned}
$$




$$
\begin{gathered}
{[H]=\left[\begin{array}{cccccc}
0 & 0 & 0 & 0 & \ldots \\
0 & 1 / 2 & 0 & 0 & \ddots & \\
0 & 0 & 2 / 2 & 0 & \ddots & \\
0 & 0 & 0 & 3 / 2 & \ddots & \\
{[G]} & =\left[\begin{array}{cccccc}
0 & \vdots & \vdots & \vdots & \ddots
\end{array}\right] \\
0 & 0 & 1 / 4 & 0 & 0 & \ldots \\
0 & -1 / 4 & 0 & 1 / 4 & 0 & \ldots \\
0 & 0 & -1 / 4 & 0 & 1 / 4 & \ldots \\
0 & 0 & 0 & -1 / 4 & 0 & \ddots \\
\vdots & \vdots & \vdots & \ddots & \ddots & \ddots
\end{array}\right]}
\end{gathered}
$$




$$
[M]=\left[\begin{array}{ccccccc}
1 / 2 & 0 & -1 / 4 & 0 & 0 & 0 & \ldots \\
0 & 1 / 16 & 0 & -1 / 16 & 0 & 0 & \ldots \\
-1 / 4 & 0 & 1 / 6 & 0 & -1 / 24 & 0 & \ddots \\
0 & -1 / 16 & 0 & 3 / 32 & 0 & -1 / 32 & \ddots \\
0 & 0 & -1 / 24 & 0 & 1 / 15 & 0 & \ddots \\
0 & 0 & 0 & -1 / 32 & 0 & 5 / 96 & \ddots \\
\vdots & \vdots & \vdots & \ddots & \ddots & \ddots & \ddots
\end{array}\right]
$$

In the above equations $f$ is a reversed flow parameter. Typical choices for $f$ in rotor work are [12]:

$$
\begin{aligned}
& f=1 \quad \text { (reversed flow neglected) } \\
& f=u_{0} /\left|u_{0}\right|=\operatorname{sgn}\left(u_{0}\right) \quad(\text { full reversed flow) } \\
& f=u_{0} / \sqrt{u_{0}^{2}+\left(v_{0}+\dot{h}_{0}-\lambda_{0}\right)^{2}}=\cos \alpha \quad \text { (weak reversed flow) }
\end{aligned}
$$

Inflow parameters $\left\{c_{\text {inflow }}\right\},\left\{e_{\text {inflow }}\right\},\left\{f_{\text {inflow }}\right\}$, and $\left[A_{\text {inflow }}\right]$ in Eq. 2.4 are defined 
as [11]:

$$
\begin{aligned}
& {\left[A_{\text {inflow }}\right]_{N \times N}=\left[D+\{d\}\left\{b^{T}\right\}+\left\{c_{\text {inflow }}\right\}\left\{d^{T}\right\}+\frac{1}{2}\left\{c_{\text {inflow }}\right\}\left\{b^{T}\right\}\right]} \\
& \{b\}_{N \times 1}=\left\{\begin{array}{lllllll}
b_{1} & b_{2} & b_{3} & \cdots & b_{N}
\end{array}\right\}^{T} \\
& \left\{c_{\text {inflow }}\right\}_{N \times 1}=\left\{\begin{array}{lllllll}
2 & 1 & 2 / 3 & 1 / 2 & \cdots & 2 / N
\end{array}\right\}^{T} \\
& \{d\}_{N \times 1}=\left\{\begin{array}{lllllll}
1 / 2 & 0 & 0 & 0 & \cdots
\end{array}\right\}^{T} \\
& \left\{e_{\text {inflow }}\right\}_{N \times 1}=[C-G]^{T}\{1\}=\left\{\begin{array}{llllll}
f & 1 / 2 & 0 & 0 & \cdots
\end{array}\right\}^{T} \\
& \left\{f_{\text {inflow }}\right\}_{N \times 1}=[K]^{T}\{1\}=\left\{\begin{array}{lllllll}
0 & f & 2 & 3 f & 4 & \cdots
\end{array}\right\}^{T}
\end{aligned}
$$

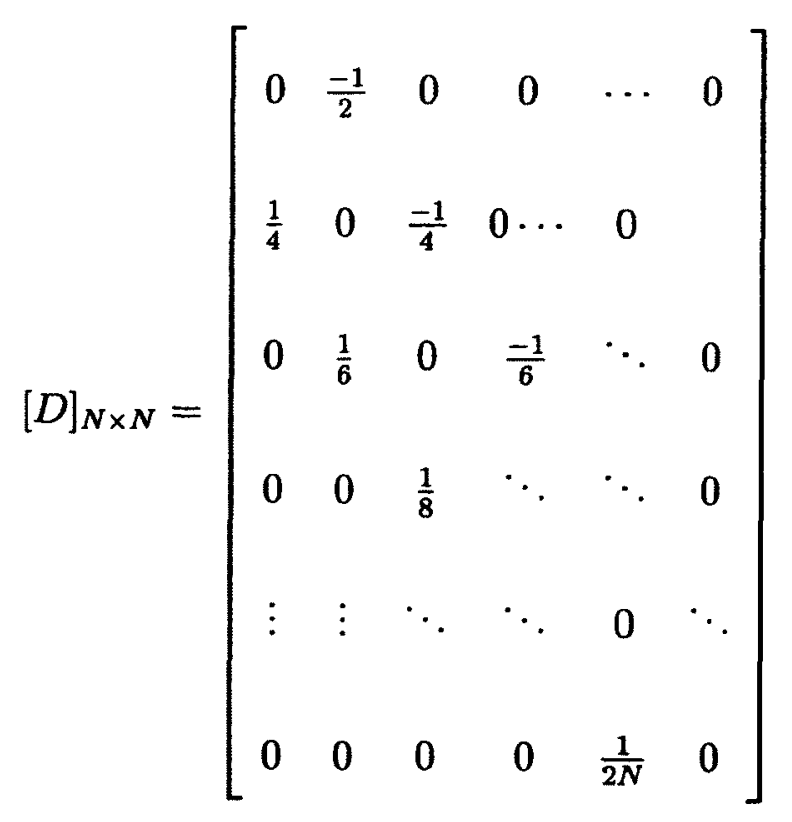




\section{Appendix B}

\section{Dynamic Modelling}

\section{B.1 Aerodynamic Coefficient Matrices}

Matrices $X_{i j}$ in Eqs. 3.32-3.34 can be obtained from the following equations:

$$
\begin{gathered}
X_{f 2 V V}=\rho b\left[\begin{array}{ccc}
0 & 0 & 0 \\
0 & -c d_{0} & 0 \\
0 & -c l_{0} & c l_{\alpha}
\end{array}\right], X_{f 2 V_{w} V}=\rho b\left[\begin{array}{ccc}
0 & 0 & 0 \\
0 & -\left(2 c d_{0}+c l_{0}\right) & 0 \\
0 & -c l_{0} & 2 c l_{\alpha}
\end{array}\right] \\
X_{f 2 V \Omega}=\rho b^{2} a\left[\begin{array}{ccc}
0 & 0 & 0 \\
-c l_{0} & 0 & 0 \\
2 c l_{\alpha} & 0 & 0
\end{array}\right], X_{f 2 \lambda \lambda}=\frac{1}{4} \rho b c l_{\alpha}\left[\begin{array}{ccc}
b_{1}^{2} & 2 b_{1} b_{2} & 2 b_{1} b_{3} \\
0 & b_{2}^{2} & 2 b_{2} b_{3} \\
0 & 0 & b_{3}^{2}
\end{array}\right]
\end{gathered}
$$




$$
X_{f 2 \Omega \Omega}=\rho b^{3} a^{2}\left[\begin{array}{ccc}
c l_{\alpha} & 0 & 0 \\
0 & 0 & 0 \\
0 & 0 & 0
\end{array}\right], X_{f 2 V \lambda}=\frac{1}{2} \rho b\left[\begin{array}{ccc}
0 & 0 & 0 \\
-b_{1} c l_{0} & -b_{2} c l_{0} & -b_{3} c l_{0} \\
2 b_{1} c l_{\alpha} & 2 b_{2} c l_{\alpha} & 2 b_{3} c l_{\alpha}
\end{array}\right]
$$

$$
X_{f 2 \Omega \lambda}=\rho b^{2} a\left[\begin{array}{ccc}
b_{1} c l_{\alpha} & b_{2} c l_{\alpha} & b_{3} c l_{\alpha} \\
0 & 0 & 0 \\
0 & 0 & 0
\end{array}\right], X_{f 3 V V}=\rho b\left[\begin{array}{ccc}
0 & 0 & 0 \\
0 & c l_{0}-\left(c l_{\alpha}+c d_{0}\right) \\
0 & 0 & 0
\end{array}\right]
$$

$$
X_{f 3 V_{w} V}=\rho b\left[\begin{array}{ccc}
0 & 0 & 0 \\
0 & 2 c l_{0} & -\left(c l_{\alpha}+c d_{0}\right) \\
0 & -\left(c l_{\alpha}+c d_{0}\right) & 0
\end{array}\right], X_{f 3 \dot{V}}=\frac{1}{2} \rho b^{2}\left[\begin{array}{c}
0 \\
0 \\
-c l_{\alpha}
\end{array}\right]^{T}
$$

$$
X_{f 3 V \Omega}=\frac{1}{2} \rho b^{2}\left[\begin{array}{ccc}
0 & 0 & 0 \\
-2 a\left(c l_{\alpha}+c d_{0}\right)+c l_{\alpha} & 0 & 0 \\
0 & 0 & 0
\end{array}\right], X_{f 3 \dot{\Omega}}=\frac{1}{2} \rho b^{3} a\left[\begin{array}{c}
-c l_{\alpha} \\
0 \\
0
\end{array}\right]^{T}
$$




$$
\begin{aligned}
& X_{f 3 V \lambda}=\frac{1}{2} \rho b\left(c l_{\alpha}+c d_{0}\right)\left[\begin{array}{ccc}
0 & 0 & 0 \\
-b_{1} & -b_{2} & -b_{3} \\
0 & 0 & 0
\end{array}\right] \\
& X_{m 1 V V}=\frac{1}{2} \rho b^{2}\left[\begin{array}{ccc}
0 & 0 & 0 \\
0 & 4 c m_{0} & -4 c m_{\alpha} \\
0 & (1+2 a) c m_{\alpha} & 4 c m_{0}
\end{array}\right]
\end{aligned}
$$

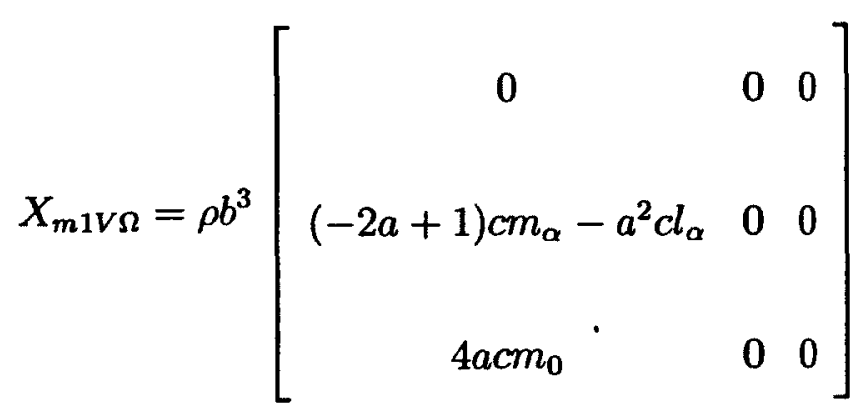

$$
\begin{aligned}
& X_{m 1 V_{w} V}=\frac{1}{2} \rho b^{2}\left[\begin{array}{ccc}
0 & 0 & 0 \\
0 & 8 c m_{0} & -4 c m_{\alpha}-(1+2 a) c l_{\alpha} \\
0 & -4 c m_{\alpha}-(1+2 a) c l_{\alpha} & 8 c m_{0}
\end{array}\right]
\end{aligned}
$$




$$
\begin{aligned}
& X_{m 1 \lambda \lambda}=\frac{1}{2} \rho b^{2}\left[\begin{array}{ccc}
b_{1}^{2} c m_{0} & b_{1} b_{2} c m_{0} & b_{1} b_{3} c m_{0} \\
0 & b_{2}^{2} c m_{0} & b_{2} b_{3} c m_{0} \\
0 & 0 & b_{3}^{2} c m_{0}
\end{array}\right] \\
& X_{m 1 \Omega \lambda}=2 \rho b^{3} a\left[\begin{array}{ccc}
b_{1} c m_{0} & b_{2} c m_{0} & b_{3} c m_{0} \\
0 & 0 & 0 \\
0 & 0 & 0
\end{array}\right] \\
& X_{m 1 V \lambda}=\frac{1}{4} \rho b^{2}\left[\begin{array}{ccc}
0 & -b_{1}\left(4 c m_{\alpha}+(1+2 a) c l_{\alpha}\right) & 8 b_{1} c m_{0} \\
0 & -b_{2}\left(4 c m_{\alpha}+(1+2 a) c l_{\alpha}\right) & 8 b_{2} c m_{0} \\
0 & -b_{3}\left(4 c m_{\alpha}+(1+2 a) c l_{\alpha}\right) & 8 b_{3} c m_{0}
\end{array}\right]^{T}
\end{aligned}
$$




$$
\begin{aligned}
& X_{m 1 \Omega \Omega}=\rho b^{4} a^{2}\left[\begin{array}{ccc}
2 c m_{0} & 0 & 0 \\
0 & 0 & 0 \\
0 & 0 & 0
\end{array}\right], X_{m 1 \dot{V}}=\frac{1}{2} \rho b^{3}\left[\begin{array}{c}
0 \\
0 \\
-2 c m_{\alpha}-a c l_{\alpha}
\end{array}\right]^{T} \\
& X_{m 1 \dot{\Omega}}=\frac{1}{2} \rho b^{4}\left[\begin{array}{c}
-2 a c m_{\alpha}-\left(a^{2}+\frac{1}{8}\right) c l_{\alpha} \\
0 \\
0
\end{array}\right]^{T}
\end{aligned}
$$




\section{Appendix $\mathbf{C}$}

\section{Galerkin Discretization}

\section{C.1 Approximation of State Variables}

The approximated form of the state variables $M, F^{a c t}, M^{a c t}, V, \Omega, g$, and $\lambda$ present in discretized equations can be presented as follows:

$$
\begin{aligned}
M(x, t) & =\sum_{i=1}^{n c} \bar{M}_{i}(x) \underline{M}_{i}(t), \\
\bar{M}_{i}(x) & =\left[\begin{array}{ccc}
\bar{M}_{1 i} & 0 & 0 \\
0 & \bar{M}_{2 i} & 0 \\
0 & 0 & \bar{M}_{3 i}
\end{array}\right], \quad \underline{M}_{i}(t)=\left\{\begin{array}{l}
\underline{M}_{1 i} \\
\underline{M}_{2 i} \\
\underline{M}_{3 i}
\end{array}\right\}
\end{aligned}
$$




$$
\begin{aligned}
F^{a c t}(x, t) & =\sum_{i=1}^{n c} \bar{F}_{i}^{a c t}(x) \underline{F}_{i}^{a c t}(t), \\
\bar{F}_{i}^{a c t}(x) & =\left[\begin{array}{ccc}
\bar{F}_{1 i}^{a c t} & 0 & 0 \\
0 & \bar{F}_{2 i}^{a c t} & 0 \\
0 & 0 & \bar{F}_{3 i}^{a c t}
\end{array}\right], \underline{F}_{i}(t)=\left\{\begin{array}{l}
\underline{F}_{1 i}^{a c t} \\
F_{2 i}^{a c t} \\
F_{3 i}^{a c t}
\end{array}\right\}
\end{aligned}
$$

$$
M^{a c t}(x, t)=\sum_{i=1}^{n c} \bar{M}_{i}^{a c t}(x) \underline{M}_{i}^{\text {act }}(t),
$$$$
\bar{M}_{i}^{a c t}(x)=\left[\begin{array}{ccc}
\bar{M}_{1 i}^{a c t} & 0 & 0 \\
0 & \bar{M}_{2 i}^{a c t} & 0 \\
0 & 0 & \bar{M}_{3 i}^{\text {act }}
\end{array}\right], \quad \underline{M}_{i}^{\text {act }}(t)=\left\{\begin{array}{c}
\underline{M}_{1 i}^{a c t} \\
\underline{M}_{2 i}^{a c t} \\
\underline{M}_{3 i}^{a c t}
\end{array}\right\}
$$$$
V(x, t)=\sum_{i=1}^{n c} \bar{V}_{i}(x) \underline{V}_{i}(t), \quad \bar{V}_{i}(x)=\left[\begin{array}{ccc}
\bar{V}_{1 i} & 0 & 0 \\
0 & \bar{V}_{2 i} & 0 \\
0 & 0 & \bar{V}_{3 i}
\end{array}\right], \quad \underline{V_{i}}(t)=\left\{\begin{array}{l}
\underline{V}_{1 i} \\
\underline{V}_{2 i} \\
\underline{V}_{3 i}
\end{array}\right\}
$$

$$
\Omega(x, t)=\sum_{i=1}^{n c} \bar{\Omega}_{i}(x) \underline{\Omega}_{i}(t), \quad \bar{\Omega}_{i}(x)=\left[\begin{array}{ccc}
\bar{\Omega}_{1 i} & 0 & 0 \\
0 & \bar{\Omega}_{2 i} & 0 \\
0 & 0 & \bar{\Omega}_{3 i}
\end{array}\right], \quad \underline{\Omega_{i}}(t)=\left\{\begin{array}{l}
\Omega_{1 i} \\
\underline{\Omega}_{2 i} \\
\underline{\Omega}_{3 i}
\end{array}\right\}
$$




$$
\begin{gathered}
g(x, t)=\sum_{i=1}^{n c} \bar{g}_{i}(x) \underline{g}_{i}(t), \quad \bar{g}_{i}(x)=\left[\begin{array}{ccc}
\bar{g}_{1 i} & 0 & 0 \\
0 & \bar{g}_{2 i} & 0 \\
0 & 0 & \bar{g}_{3 i}
\end{array}\right], \quad \underline{g}_{i}(t)=\left\{\begin{array}{l}
\underline{g}_{1 i} \\
\underline{g}_{2 i} \\
\underline{g}_{3 i}
\end{array}\right\} \\
\lambda(x, t)=\sum_{i=1}^{n c} \bar{\lambda}_{i}(x) \underline{\lambda}_{i}(t), \quad \bar{\lambda}_{i}(x)=\left[\begin{array}{ccc}
\bar{\lambda}_{1 i} & 0 & 0 \\
0 & \bar{\lambda}_{2 i} & 0 \\
0 & 0 & \bar{\lambda}_{3 i}
\end{array}\right], \quad \underline{\lambda}_{i}(t)=\left\{\begin{array}{l}
\underline{\lambda}_{1 i} \\
\underline{\lambda}_{2 i} \\
\underline{\lambda}_{3 i}
\end{array}\right\}
\end{gathered}
$$

Defining the generalized temporal function $q_{i}(t)$ and its magnitude $\left|q_{i}(t)\right|$, the approximation of the state variables can be rewritten as:

$$
\begin{aligned}
V(x, t) & =\sum_{i=1}^{n c} \Phi_{i}^{V}(x) q_{i}(t), \quad \Phi_{i}^{V}(x)=\left[\bar{V}_{i}, 0,0,0,0,0,0,0\right]_{(3 \times 24)} \\
\Omega(x, t) & =\sum_{i=1}^{n c} \Phi_{i}^{\Omega}(x) q_{i}(t), \quad \Phi_{i}^{\Omega}(x)=\left[0, \bar{\Omega}_{i}, 0,0,0,0,0\right]_{(3 \times 24)} \\
M(x, t) & =\sum_{i=1}^{n c} \Phi_{i}^{M}(x) q_{i}(t), \quad \Phi_{i}^{M}(x)=\left[0,0,0, \bar{M}_{i}, 0,0,0,0\right]_{(3 \times 24)} \\
\lambda(x, t) & =\sum_{i=1}^{n c} \Phi_{i}^{\lambda}(x) q_{i}(t), \quad \Phi_{i}^{\lambda}(x)=\left[0,0,0,0, \bar{\lambda}_{i}, 0,0,0\right]_{(3 \times 24)} \\
g(x, t) & =\sum_{i=1}^{n c} \Phi_{i}^{g}(x) q_{i}(t), \quad \Phi_{i}^{g}(x)=\left[0,0,0,0,0, \bar{g}_{i}, 0,0\right]_{(3 \times 24)} \\
F^{a c t}(x, t) & =\sum_{i=1}^{n c} \Phi_{i}^{F^{a c t}}(x) q_{i}(t), \quad \Phi_{i}^{F^{a c t}}(x)=\left[0,0,0,0,0,0, \bar{F}_{i}^{a c t}, 0\right]_{(3 \times 24)} \\
M^{a c t}(x, t) & =\sum_{i=1}^{n c} \Phi_{i}^{M^{a c t}}(x) q_{i}(t), \Phi_{i}^{M^{a c t}}(x)=\left[0,0,0,0,0,0,0, \bar{M}_{i}^{a c t}\right]_{(3 \times 24)}
\end{aligned}
$$




\section{C.2 Matrices and Vectors Used in Discretization}

Unless otherwise stated, all the following matrices that are used for discretization have dimensions $3 \times 24$.

$$
\begin{aligned}
& e_{1}^{V}=\left[\begin{array}{ccccc}
1 & 1 & 1 & & \\
0 & 0 & 0 & 0_{(3 \times 3)} & 0_{(3 \times 18)} \\
0 & 0 & 0 & &
\end{array}\right] \\
& e_{2}^{V}=\left[\begin{array}{llllll}
0 & 0 & 0 & & \\
1 & 1 & 1 & 0_{(3 \times 3)} & & 0_{(3 \times 18)} \\
0 & 0 & 0 & &
\end{array}\right]
\end{aligned}
$$

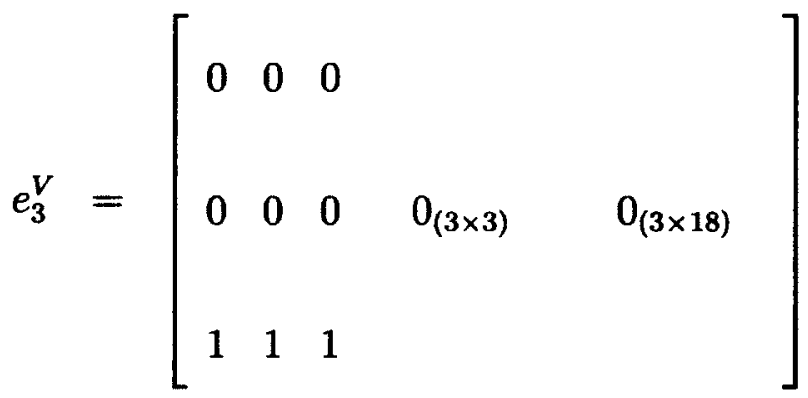

$$
\begin{aligned}
& e^{V}=\left[\begin{array}{llllll}
1 & 1 & 1 & & \\
1 & 1 & 1 & 0_{(3 \times 3)} & 0_{(3 \times 18)} \\
1 & 1 & 1 & &
\end{array}\right]
\end{aligned}
$$




\begin{tabular}{|c|c|c|c|c|c|}
\hline \multirow{3}{*}{$e_{1}^{\Omega}=$} & & 1 & 1 & 1 & \multirow{3}{*}{$0_{(3 \times 18)}$} \\
\hline & $0_{(3 \times 3)}$ & 0 & 0 & 0 & \\
\hline & & 0 & 0 & 0 & \\
\hline \multirow{3}{*}{$e_{2}^{\Omega}=$} & & 0 & 0 & 0 & \\
\hline & $0_{(3 \times 3)}$ & 1 & 1 & 1 & $0_{(3 \times 18)}$ \\
\hline & & 0 & 0 & 0 & \\
\hline \multirow{3}{*}{$e_{3}^{\Omega}=$} & & 0 & 0 & 0 & \\
\hline & $0_{(3 \times 3)}$ & 0 & 0 & 0 & $0_{(3 \times 18)}$ \\
\hline & & 1 & 1 & 1 & \\
\hline \multirow{3}{*}{$e^{\Omega}=$} & & 1 & 1 & 1 & \\
\hline & $0_{(3 \times 3)}$ & 1 & 1 & 1 & $0_{(3 \times 6)}$ \\
\hline & & 1 & 1 & 1 & \\
\hline
\end{tabular}




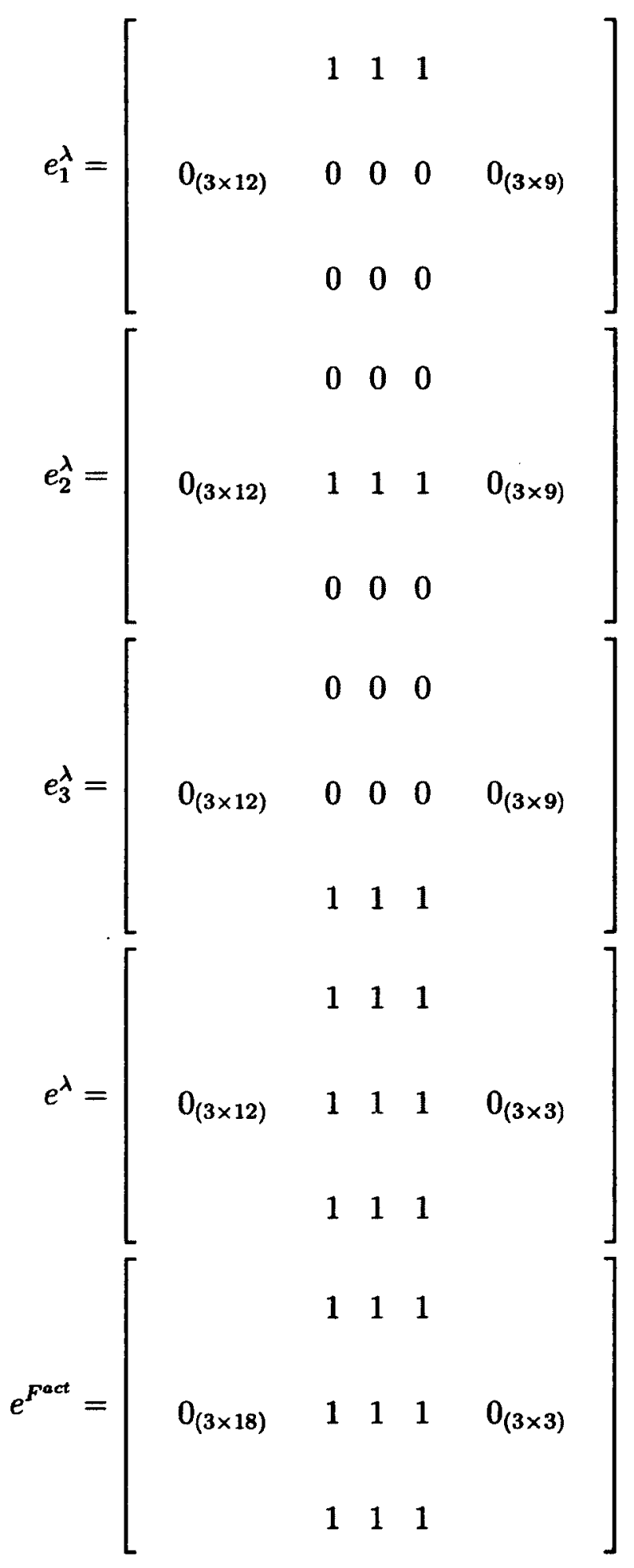




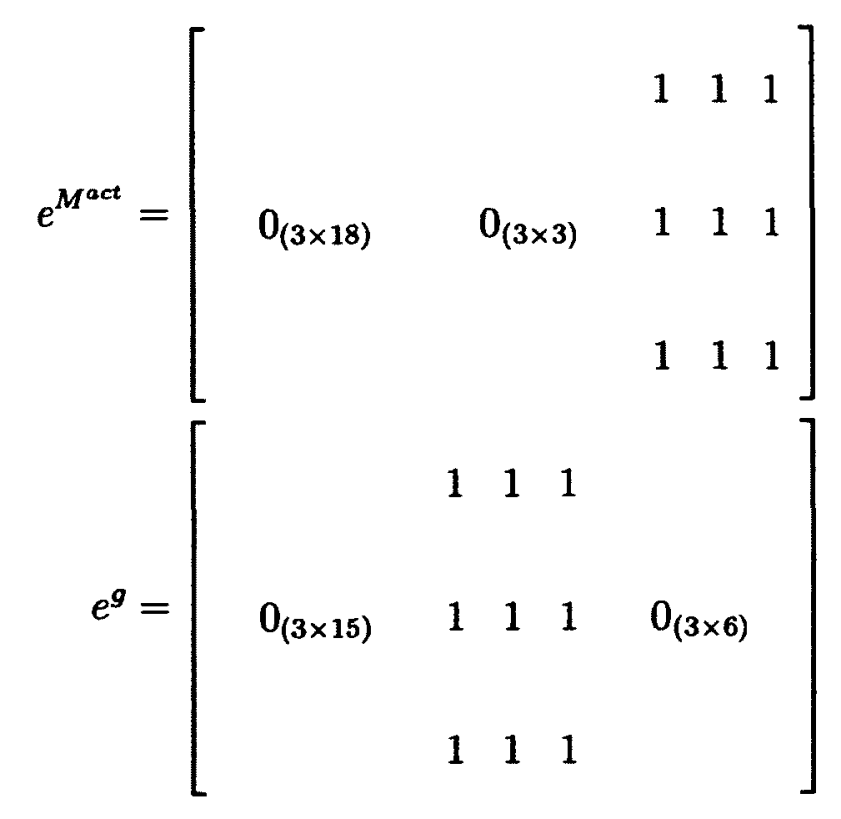

$$
\begin{aligned}
\Phi_{l}^{\left|V^{T} G g_{m}\right|}= & \left\{\left|\bar{V}_{1 l}\right| g_{m}\left(G_{11}^{2}+G_{12}^{2}+G_{13}^{2}\right)^{\left(\frac{1}{2}\right)},\left|\bar{V}_{2 l}\right| g_{m}\left(G_{21}^{2}+G_{22}^{2}+G_{23}^{2}\right)^{\left(\frac{1}{2}\right)}\right. \\
& \left.\left|\bar{V}_{3 l}\right| g_{m}\left(G_{31}^{2}+G_{32}^{2}+G_{33}^{2}\right)^{\left(\frac{1}{2}\right)}, 0_{(1 \times 21)}\right\}_{(24 \times 1)}^{T}
\end{aligned}
$$$$
\Phi_{l}^{\left|\Omega^{T} Q g_{m}\right|}=\left\{0_{(1 \times 3)},\left|\bar{\Omega}_{1 l}\right| g_{m}\left(Q_{11}^{2}+Q_{12}^{2}+Q_{13}^{2}\right)^{\left(\frac{1}{2}\right)},\left|\bar{\Omega}_{2 l}\right| g_{m}\left(Q_{21}^{2}+Q_{22}^{2}+Q_{23}^{2}\right)^{\left(\frac{1}{2}\right)}\right.
$$$$
\left.\left|\bar{\Omega}_{3 l}\right| g_{m}\left(Q_{31}^{2}+Q_{32}^{2}+Q_{33}^{2}\right)^{\left(\frac{1}{2}\right)}, 0_{(1 \times 18)}\right\}_{(24 \times 1)}^{T}
$$

$$
\Phi_{i}^{|g|}=\left\{0,0,0,0,0,\left|\overline{g_{i}}\right|, 0,0\right\}_{(1 \times 9)}
$$




$$
\begin{aligned}
& \Phi_{l}^{V}(x)=\Psi^{V} P_{l}(x), \quad \Psi^{V}=[I, 0,0,0,0,0,0,0]_{(3 \times 24)} \\
& \Phi_{l}^{\Omega}(x)=\Psi^{\Omega} P_{l}(x), \quad \Psi^{\Omega}=[0, I, 0,0,0,0,0,0]_{(3 \times 24)} \\
& \Phi_{l}^{M}(x)=\Psi^{M} P_{l}(x), \quad \Psi^{M}=[0,0,0, I, 0,0,0,0]_{(3 \times 24)} \\
& \Phi_{l}^{\lambda}(x)=\Psi^{\lambda} P_{l}(x), \quad \Psi^{\lambda}=[0,0,0,0, I, 0,0,0]_{(3 \times 24)} \\
& \Phi_{l}^{g}(x)=\Psi^{g} P_{l}(x), \quad \Psi^{g}=[0,0,0,0,0, I, 0,0]_{(3 \times 24)} \\
& \Phi_{l}^{F^{a c t}}(x)=\Psi^{F^{a c t}} P_{l}(x), \quad \Psi^{F^{a c t}}=[0,0,0,0,0,0, I, 0]_{(3 \times 24)} \\
& \Phi_{l}^{M^{a c t}}(x)=\Psi^{M^{a c t}} P_{l}(x), \quad \Psi^{M^{a c t}}=[0,0,0,0,0,0,0, I]_{(3 \times 24)} \\
& \Phi_{l}^{U}(x)=\Psi^{U} P_{l}(x), \quad \Psi^{U}=I_{(3 \times 3)} \\
& \Phi_{l}^{C^{B b}}(x)=\Psi^{C^{B b}} P_{l}(x), \quad \Psi^{C^{B b}}=I_{(3 \times 3)} \\
& \Phi_{l}^{\left|V^{T} G g_{m}\right|}=\Psi_{l}^{\left|V^{T} G g_{m}\right|}\left|P_{l}(x)\right| \\
& \Psi_{l}^{\left|V^{T} G g_{m}\right|}=\left\{g_{m}\left(G_{11}^{2}+G_{12}^{2}+G_{13}^{2}\right)^{\left(\frac{1}{2}\right)}, g_{m}\left(G_{21}^{2}+G_{22}^{2}+G_{23}^{2}\right)^{\left(\frac{1}{2}\right)},\right. \\
& \left.g_{m}\left(G_{31}^{2}+G_{32}^{2}+G_{33}^{2}\right)^{\left(\frac{1}{2}\right)}, 0_{(1 \times 21)}\right\}_{(24 \times 1)}^{T} \\
& \Phi_{l}^{\left|\Omega^{T} Q g_{m}\right|}=\Psi_{l}^{\left|\Omega^{T} Q g_{n n}\right|}\left|P_{l}(x)\right| \\
& \Psi_{l}^{\left|\Omega^{T} Q g_{m}\right|}=\left\{0_{(1 \times 3)}, g_{m}\left(Q_{11}^{2}+Q_{12}^{2}+Q_{13}^{2}\right)^{\left(\frac{1}{2}\right)}, g_{m}\left(Q_{21}^{2}+Q_{22}^{2}+Q_{23}^{2}\right)^{\left(\frac{1}{2}\right)}\right. \\
& \left.g_{m}\left(Q_{31}^{2}+Q_{32}^{2}+Q_{33}^{2}\right)^{\left(\frac{1}{2}\right)}, 0_{(1 \times 18)}\right\}_{(24 \times 1)}^{T} \\
& \Phi_{i}^{|g|}=\Psi_{i}^{|g|}\left|P_{l}(x)\right|, \quad \Psi_{i}^{|g|}=\{0,0,0,0,0, \sqrt{3}, 0,0\}_{(1 \times 8)}
\end{aligned}
$$

